\title{
SOFTWARE-DEFINED UNDERWATER ACOUSTIC MODEMS FOR ARCTIC-LIKE ENVIRONMENTS
}

\author{
by \\ Ahmad Traboulsi \\ A thesis submitted to \\ the Faculty of Graduate Studies and Research \\ in partial fulfillment of \\ the requirements for the degree of \\ MASTER OF SCIENCE \\ School of Computer Science \\ at \\ CARLETON UNIVERSITY
}

Ottawa, Ontario

September, 2016

(c) Copyright by Ahmad Traboulsi, 2016 


\section{Table of Contents}

List of Tables $\quad$ iv

List of Figures $\quad$ V

Abstract $\quad$ xiii

Acknowledgements $\quad$ xiv

Chapter 1 Introduction 1

1.1 Underwater Communications . . . . . . . . . . . . . . . . . 2

1.2 Statement of the Problem . . . . . . . . . . . . . 5

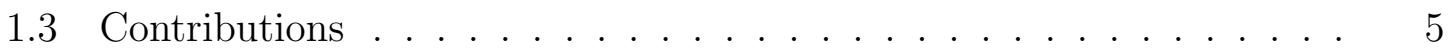

1.4 Organization of Thesis . . . . . . . . . . . . . 6

$\begin{array}{lll}\text { Chapter } 2 & \text { Background } & 8\end{array}$

2.1 Underwater Acoustics . . . . . . . . . . . . . . . . . 8

2.2 Software-Defined Communication . . . . . . . . . . . . . 14

2.2.1 Software-Defined Communication Systems . . . . . . . . . . 14

2.2.2 GNU Radio . . . . . . . . . . . . . . . . . . . . 15

$\begin{array}{lll}\text { Chapter } 3 & \text { Related Work } & 17\end{array}$

3.1 Underwater Communication Modems . . . . . . . . . . . . . 17

Chapter 4 Modem Design 22

4.1 Introduction . . . . . . . . . . . . . . . . . . 22

4.2 Single-Carrier Modulation . . . . . . . . . . . . . . . . . 23

4.2.1 Phase-Shift Keying Modulation . . . . . . . . . . . . . 23

4.2.2 Frequency-Shift Keying Modulation . . . . . . . . . . . . . 32

4.3 Orthogonal Frequency-Division Multiplexing . . . . . . . . . . 34

4.4 Frequency Hopping-Continuous-Phase Frequency-Shift Keying . . . . 38 
$\begin{array}{lll}\text { Chapter } 5 & \text { Simulation and Results } & 42\end{array}$

5.1 Introduction . . . . . . . . . . . . . . . . . . . . . . . . . . 42

5.2 Simulation Methodology . . . . . . . . . . . . . . . . . 42

5.3 Impulse Response . . . . . . . . . . . . . . . . . . . . . . . 43

5.4 Simulation Environments . . . . . . . . . . . . . . . . . 45

5.5 Measure of Variability . . . . . . . . . . . . . . . . . . . . . 49

5.6 Delay Spreads . . . . . . . . . . . . . . . . . . . . . . . . . . . . 52

5.7 Results . . . . . . . . . . . . . . . . . . . . . . . 55

5.7.1 Binary Phase-Shift Keying Performance . . . . . . . . . 55

$5.7 .2 \quad$ Frequency-Shift Keying Performance . . . . . . . . . . . . 59

5.7.3 Frequency Hopping-Continuous Phase-Shift Keying Performance 62

5.7.4 Orthogonal Frequency-Division Multiplexing Performance. . . 65

5.7.5 Comparison of the Performance of Various Modulation Techniques . . . . . . . . . . . . . . . . 67

5.8 Summary . . . . . . . . . . . . . . . . . . . . 78

$\begin{array}{lll}\text { Chapter } 6 & \text { Conclusion } & 79\end{array}$

6.1 Summary of Work . . . . . . . . . . . . . . . . . 79

6.2 Future Work . . . . . . . . . . . . . . . . . . . . . 80

$\begin{array}{ll}\text { Appendix A } & 81\end{array}$

$\begin{array}{ll}\text { Bibliography } & 93\end{array}$ 


\section{List of Tables}

$5.1 \quad$ Summary of Simulations. . . . . . . . . . . . 78

A.1 Summary of Simulations Variables. . . . . . . . . . . . 92 


\section{List of Figures}

$1 \quad$ An underwater network consisting of stationary and mobile nodes. 2

2 Example of reflected and direct paths. . . . . . . . . 9

3 Refraction $\left(C_{1}\right.$ is larger than $\left.C_{2}\right) \ldots \ldots \ldots 11$

$4 \quad$ An example of a sound speed profile. . . . . . . . . . . . . . . 12

$5 \quad$ Eigenrays generated in an environment with refracted paths (in red) and reflected paths (in black). . . . . . . . . . . . . 12

$6 \quad$ Single path vs multipath propagation between a transmitter $(\mathrm{TX})$ and a receiver $(\mathrm{RX}) \ldots \ldots \ldots . \ldots . \ldots 13$

A GNU Radio flowgraph. . . . . . . . . . . . . . . . . 16

Time domain view of a DPSK signal. . . . . . . . . . . 23

BPSK and QPSK constellations . . . . . . . . . . 24

10 BPSK transmitter module. . . . . . . . . . . 25

11 Mitigating channel effects with PSK single-carrier modulation. 26

12 BPSK sampling point with raised cosine filter. . . . . . . . . 27

13 BPSK receiver module. . . . . . . . . . . . . . . 29

14 FLL Band-Edge. . . . . . . . . . . . . . . 30

$15 \quad$ Finding the optimal sampling point using a bank of differential filters. . . . . . . . . . . . . . . 31

$16 \quad$ FSK waveform example. . . . . . . . . . . . . . . 32

17 FSK transmitter module. . . . . . . . . . . . . 33

$18 \quad$ FSK receiver module. . . . . . . . . . . . . . 33

19 OFDM transmitter module. . . . . . . . . . 35

20 OFDM receiver module. . . . . . . . . . . . . 36

$21 \quad$ Frequency Hopping CPFSK. . . . . . . . . . . 39

22 Frequency Hopping CPFSK transmitter module. . . . . . . 40

23 Frequency Hopping CPFSK receiver module. . . . . . . . . . 41 
Architecture of the simulator. . . . . . . . . . . . . . 43

An example of an environment file. . . . . . . . . . . . 44

Upward refracting sound speed profile. . . . . . . . . . 46

Environment sample with $0 \%$ ice cover. . . . . . . . . 46

28 Environment sample with $20 \%$ ice cover. . . . . . . . . . 47

Environment sample with $40 \%$ ice cover. . . . . . . . . . 47

$30 \quad$ Environment sample with $80 \%$ ice cover. . . . . . . . . . 48

31 Environment sample with $20 \%$ ice cover and $10 \mathrm{~km}$ range. . . 48

32 IQR for 100 files with 20 percent ice cover and $5 \mathrm{~km}$ in range. 49

33 IQR for 100 files with 40 percent ice cover and $5 \mathrm{~km}$ in range. $\quad 50$

$34 \quad$ IQR for 100 files with 80 percent ice cover and $5 \mathrm{~km}$ in range. $\quad 50$

35 IQR for 100 files with 20 percent ice cover and $10 \mathrm{~km}$ in range. 51

36 The delay spread of each of the 100 files with $20 \%$ ice cover and $5 \mathrm{~km}$ in range. The average of the delay spreads equals to 0.4446, the standard deviation equals to 0.0884 and the median equals to $0.4336 . \ldots \ldots \ldots$. . . . . . . . . . . . 52

37 The delay spread of each of the 100 files with $40 \%$ ice cover and $5 \mathrm{~km}$ in range. The average of the delay spreads equals to 0.4552 , the standard deviation equals to 0.0841 and the median equals to $0.4458 . \ldots \ldots \ldots \ldots$. . . . . . . . . . 53

38 The delay spread of each of the 100 files with $80 \%$ ice cover and $5 \mathrm{~km}$ in range. The average of the delay spreads after removing the outlier equals to 0.5034 , the standard deviation equals to 0.0861 and the median equals to 0.4949 . . . . . . . . . .

The delay spread of each of the 100 files with $20 \%$ ice cover and $10 \mathrm{~km}$ in range. The average of the delay spreads equals to 0.6670 , the standard deviation equals to 0.1032 and the median equals to $0.6639 . \ldots \ldots \ldots \ldots \ldots$ BPSK with different symbol times over a range of $5 \mathrm{~km}$ and $20 \%$ of ice cover. . . . . . . . . . . . . . . . 55 
41 Confidence interval with $90 \%$ confidence level. BPSK with $20 \%$ of ice cover in $5 \mathrm{~km}$ range. The Figure shows three different symbol times used. . . . . . . . . . . . . . . 56

$42 \quad$ BPSK with different ice cover percentage over a range of $5 \mathrm{~km}$ using a symbol time of three seconds. . . . . . . . . . . . 57

43 BPSK with $20 \%$ of ice cover but two different ranges and same symbol time of three seconds . . . . . . . . . . . . . . 57

44 Confidence interval with $90 \%$ confidence level. BPSK with $20 \%$, $40 \%, 80 \%$ of ice cover in $5 \mathrm{~km}$ range and $20 \%$ ice cover in 10 $\mathrm{km}$ range. Symbol time is equals to three seconds. . . . . . . . 58

$45 \quad 2$-FSK with different symbol times over a range of $5 \mathrm{~km}$ and $20 \%$ of ice cover. . . . . . . . . . . . . . . . 59

$46 \quad$ Confidence interval with $90 \%$ confidence level. 2-FSK with $20 \%$ of ice cover in $5 \mathrm{~km}$ range. The Figure shows three different symbol times used. . . . . . . . . . . . . . . . . 59

$47 \quad$ 2-FSK with different ice cover percentages over a range of $5 \mathrm{~km}$ using a symbol time of three seconds. . . . . . . . . . . 60

48 2-FSK with $20 \%$ of ice cover, two different ranges and same symbol time of three seconds. . . . . . . . . . . . . . . 61

49 Confidence interval with $90 \%$ confidence level. 2-FSK with $20 \%$, $40 \%, 80 \%$ of ice cover in $5 \mathrm{~km}$ range and $20 \%$ ice cover in 10 $\mathrm{km}$ range. Symbol time is equals to three seconds. . . . . . . . 61

$50 \quad$ FH-CPFSK with different symbol times over a range of $5 \mathrm{~km}$.

51 Confidence interval with $90 \%$ confidence level. FH-CPFSK with $20 \%$ of ice cover in $5 \mathrm{~km}$ range. The Figure shows three different symbol times used. . . . . . . . . . . . . . . . . 62

$52 \quad$ FH-CPFSK with different ice cover percentages over a range of $5 \mathrm{~km}$ using a symbol time of three seconds. . . . . . . . . . 63

53 FH-CPFSK with $20 \%$ of ice cover, two different ranges and symbol time of three seconds. . . . . . . . . . . . . . . . 64 
$54 \quad$ Confidence interval with $90 \%$ confidence level. FH-CPFSK with $20 \%, 40 \%, 80 \%$ of ice cover in $5 \mathrm{~km}$ range and $20 \%$ ice cover in $10 \mathrm{~km}$ range. Symbol time is equals to three seconds. . . . . . OFDM with different packet sizes and symbol times over a range of $5 \mathrm{~km} \ldots \ldots \ldots \ldots \ldots \ldots \ldots \ldots$

$56 \quad$ O4Bp OFDM with different ice cover percentages over a range of $5 \mathrm{~km}$ using a symbol time of three seconds. . . . . . . . 66

57 BPSK vs 2-FSK vs FH-CPFSK in $0 \%$ of ice cover over a range of $5 \mathrm{~km} \ldots \ldots \ldots \ldots \ldots \ldots \ldots \ldots$

58 BPSK vs 2-FSK vs FH-CPFSK in $20 \%$ of ice cover over a range of $5 \mathrm{~km}$ environment. . . . . . . . . . . . . . . . . 68

59 Confidence interval with $90 \%$ confidence level. BPSK vs FSK vs FH-CFSK in $20 \%$ of ice cover over a range of $5 \mathrm{~km}$ environment. 68 60 BPSK vs 2-FSK vs FH-CPFSK in $40 \%$ of ice cover over a range of $5 \mathrm{~km}$ environment. . . . . . . . . . . . . . . . 69

61 Confidence interval with $90 \%$ confidence level. BPSK vs FSK vs FH-CFSK in $40 \%$ of ice cover over a range of $5 \mathrm{~km}$ environment. 69 BPSK vs 2-FSK vs FH-CPFSK in $80 \%$ of ice cover over a range of $5 \mathrm{~km}$ environment. . . . . . . . . . . . . . . 70

63 Confidence interval with $90 \%$ confidence level. BPSK vs FSK vs FH-CFSK in $80 \%$ of ice cover over a range of $5 \mathrm{~km}$ environment. 70 BPSK vs 2-FSK vs FH-CPFSK in $20 \%$ of ice cover over a range of $10 \mathrm{~km}$ environment. . . . . . . . . . . . . . . . 71

65 Confidence interval with $90 \%$ confidence level. BPSK vs FSK vs FH-CFSK in $20 \%$ of ice cover over a range of $10 \mathrm{~km}$ environment. 72 PER vs SNR of BPSK vs 2-FSK vs FH-CPFSK vs O4Bp OFDM in $0 \%$ of ice cover over a range of $5 \mathrm{~km}$ environment. . . . . 73 PER vs SNR of BPSK vs 2-FSK vs FH-CPFSK vs O4Bp OFDM in $20 \%$ of ice cover over a range of $5 \mathrm{~km}$ environment. . . . . . 74 
71 Confidence interval with $90 \%$ confidence level. BPSK with $20 \%$ of ice cover, $5 \mathrm{~km}$ range and symbol time of three seconds. . . 81

72 Confidence interval with $90 \%$ confidence level. BPSK with $40 \%$ of ice cover, $5 \mathrm{~km}$ range and symbol time of three seconds. . . 82

73 Confidence interval with $90 \%$ confidence level. BPSK with $80 \%$ of ice cover, $5 \mathrm{~km}$ range and symbol time of three seconds. . . 82

$74 \quad$ Confidence interval with $90 \%$ confidence level. BPSK with $20 \%$ of ice cover, $10 \mathrm{~km}$ range and symbol time of three seconds. . .

75 Confidence interval with $90 \%$ confidence level. 2-FSK with $20 \%$ of ice cover, $5 \mathrm{~km}$ range and symbol time of three seconds. . . 84

76 Confidence interval with $90 \%$ confidence level. 2-FSK with $40 \%$ of ice cover, $5 \mathrm{~km}$ range and symbol time of three seconds. . . 84

77 Confidence interval with $90 \%$ confidence level. 2-FSK with $80 \%$ of ice cover, $5 \mathrm{~km}$ range and symbol time of three seconds. . . 85

78 Confidence interval with $90 \%$ confidence level. 2-FSK with $20 \%$ of ice cover, $10 \mathrm{~km}$ range and symbol time of three seconds. . .

79 Confidence interval with $90 \%$ confidence level. FH-CPFSK with $20 \%$ of ice cover, $5 \mathrm{~km}$ range and symbol time of three seconds. 86

80 Confidence interval with $90 \%$ confidence level. FH-CPFSK with $40 \%$ of ice cover, $5 \mathrm{~km}$ range and symbol time of three seconds. 86 Confidence interval with $90 \%$ confidence level. FH-CPFSK with $80 \%$ of ice cover, $5 \mathrm{~km}$ range and symbol time of three seconds. 87 
82 Confidence interval with $90 \%$ confidence level. FH-CPFSK with $20 \%$ of ice cover, $10 \mathrm{~km}$ range and symbol time of three seconds. 87

83 Confidence interval with $90 \%$ confidence level. O4Bp OFDM with $20 \%$ of ice cover and $5 \mathrm{~km}$ range. . . . . . . . . 88

84 Confidence interval with $90 \%$ confidence level. O4Bp OFDM with $40 \%$ of ice cover and $5 \mathrm{~km}$ range. . . . . . . . . 88

85 Confidence interval with $90 \%$ confidence level. O4Bp OFDM with $80 \%$ of ice cover and $5 \mathrm{~km}$ range. . . . . . . . . . 89

86 Confidence interval with $90 \%$ confidence level. O4Bp OFDM with $20 \%$ of ice cover and $10 \mathrm{~km}$ range. . . . . . . . . . 89

87 Confidence interval with $90 \%$ confidence level. BPSK vs FSK vs FH-CFSK vs O4Bp OFDM in $20 \%$ of ice cover over a range of $5 \mathrm{~km}$ environment. . . . . . . . . . . . . . . . . 90

88 Confidence interval with $90 \%$ confidence level. BPSK vs FSK vs FH-CFSK vs O4Bp OFDM in $40 \%$ of ice cover over a range of $5 \mathrm{~km}$ environment. . . . . . . . . . . . . . . . . 90

89 Confidence interval with $90 \%$ confidence level. BPSK vs FSK vs $\mathrm{FH}-\mathrm{CFSK}$ vs O4Bp OFDM in $80 \%$ of ice cover over a range of $5 \mathrm{~km}$ environment. . . . . . . . . . . . . . . . . 91

90 Confidence interval with $90 \%$ confidence level. BPSK vs FSK vs FH-CFSK vs O4Bp OFDM in $20 \%$ of ice cover over a range of $10 \mathrm{~km}$ environment. . . . . . . . . . . . . . . . . 91 


\section{Glossary}

ASK Amplitude-Shift Keying.

DPSK Differential Phase-Shift Keying.

DSP Digital Signal Processing.

DSSS Direct Sequency Spread Spectrum.

$E_{b} / N_{0}$ Energy per Bit to Noise Power Spectral Density Ratio.

FDM Frequency-Division Multiplexing.

FIR Finite Impulse Response.

FLL Frequency-Locked Loop.

FPGA Field-Programmable Gate Array.

FRSS Frequency Repetition Spread Spectrum.

GMSK Gaussian Minimum-Shift Keying.

IFFT Inverse Fast Fourier Transform.

LFM Linear Frequency Modulated.

PN Pseudo Random Noise.

QPSK Quadrature Phase-Shift Keying.

SINR Signal-to-Interference-plus-Noise-Ratio.

$T_{s}$ Symbol Time.

BER Bit Error Rate.

BPSK Binary Phase-Shift Keying. 
FH-CPFSK Frequency Hopping Continuous Phase Frequency-Shift Keying.

FSK Frequency-Shift Keying.

ISI Inter-Symbol Interference.

OFDM Orthogonal Frequency-Division Multiplexing.

PDP Power Delay Profile.

PER Packet Error Rate.

RF Radio Frequency.

RMS Root Mean Squared.

SDAC Software Defined Acoustic Communication.

SDR Software-Defined Radio.

SNR Signal to Noise Ratio. 


\begin{abstract}
A wide range of underwater sensor network applications have developed in the recent years. They include applications such as surveillance of coastal water in the Arctic. Underwater communications in Arctic-like environments is challenging. The ice cover in an upward refracting environment creates substantial signal scattering. We simulate the underwater Arctic condition for BPSK, FSK, FH-CPFSK and OFDM signals. We present the results of the performance analysis for these modulation schemes and transmission techniques. The simulated conditions consist of $0 \%, 20 \%, 40 \%$ and $80 \%$ of ice cover over a distance of $5 \mathrm{~km}$ and $20 \%$ of ice cover over a distance of $10 \mathrm{~km}$ with upward refraction. We concluded that FSK and FH-CPFSK are more efficient than BPSK and OFDM. We observed that at lower levels of $E_{b} / N_{0}$, FH-CPFSK performs better and on higher levels of $E_{b} / N_{0}$ FSK performs better. In the absence of ice cover FH-CPFSK outperforms FSK.
\end{abstract}




\section{Acknowledgements}

I would like to thank my thesis supervisor Professor Michel Barbeau for the guidance and the passing of the required knowledge to me to have this thesis done. I would also like to thank my co-supervisor Doctor Stéphane Blouin for the notes and directions given to me during our discussions and meetings. I would also like to thank Mr. Craig Hamm for his cooperation and guidance on this project, moreover to sharing the material and software needed to have the simulations completed. Financial support from Maritime Way Scientific Ltd ., Natural Sciences and Engineering Research Council of Canada (NSERC), Ontario Centres of Excellence (OCE) and Public Works and Government Services Canada (PWGSC) is acknowledged. 


\section{Chapter 1}

\section{Introduction}

Underwater communications is about techniques for sending and receiving messages under sea surface. These include wireless communications using acoustic, Radio Frequency $(\mathrm{RF})$ or optical waves and wired connection using fiber optic or copper cables. Acoustic waves are generated by a mechanical vibration at a certain frequency. Because of the elasticity of water, acoustic waves create pressure differentials. The detection of the chanes in acoustic pressure is done using a hydrophone, which is an underwater microphone. Acoustic waves are used for underwater wireless communications rather than RF or optical waves due to several reasons discussed further in Section 1.1. Underwater communications are used to build underwater sensor networks that are used in many scientific, industrial and defense applications. An underwater sensor network can consist of moving and stationary nodes as shown in Figure 1. The use of underwater unmanned gliders in Arctic-like conditions that can operate for months has been discussed by Jones et al. [1]. A long range acoustic navigation and communication system was developed and used in underwater unmanned vehicles in Arctic-like conditions [2].

Establishing underwater communications require acoustic modems. Commercial acoustic modems are available $[3,4,5,6]$. Their proprietary nature makes them difficult to use for experimentation in an academic environment.

The first section in this chapter introduces some of the underwater communications applications, justifies the use of underwater acoustic communications, and gives a definition of the software-defined modem concept. Section 2 of this chapter defines the problem associated to underwater communications. The third section summarizes the contribution and work done in this thesis. Finally, the last section of this chapter 
gives a brief description on the organization of this thesis.

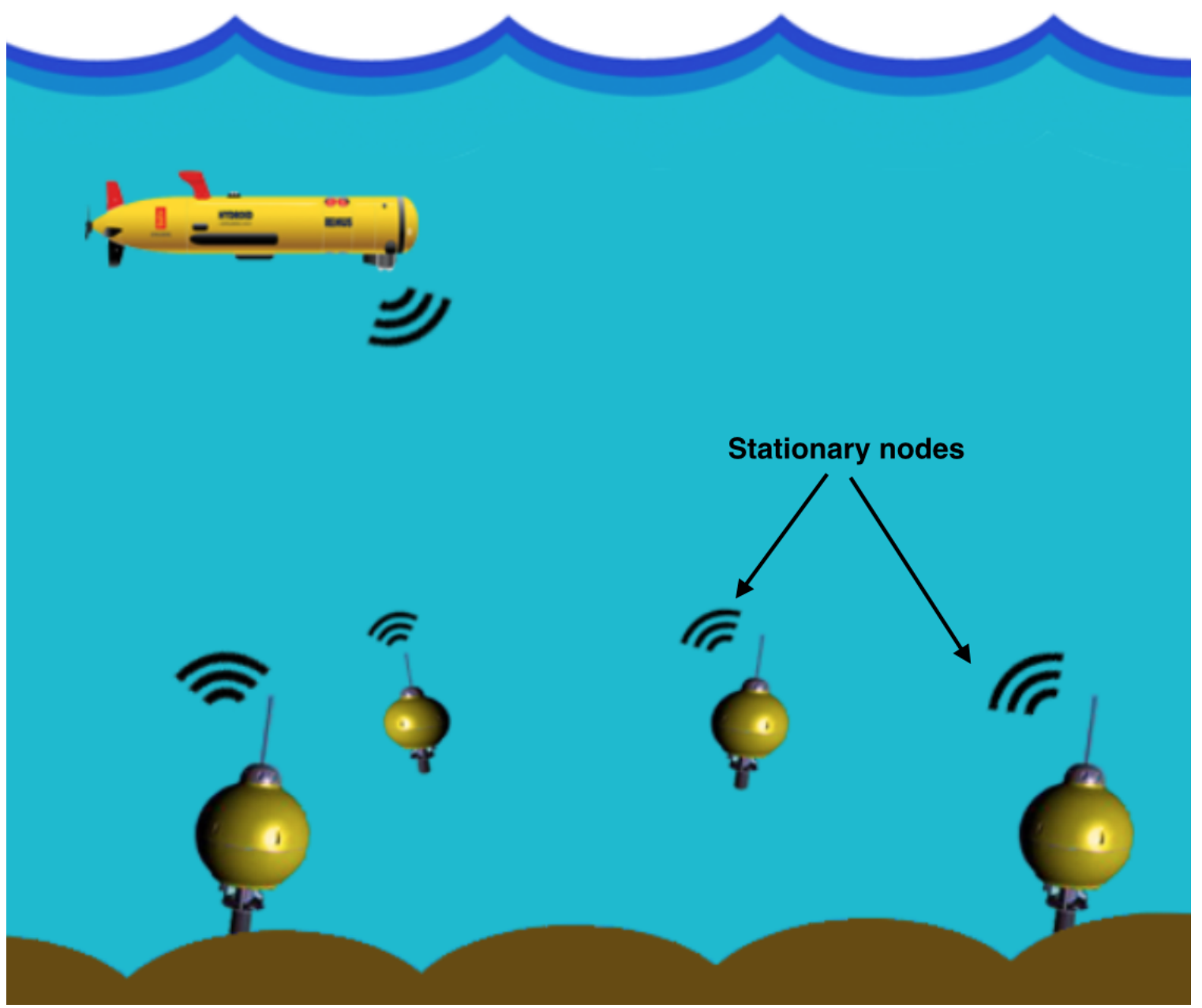

Figure 1: An underwater network consisting of stationary and mobile nodes.

\subsection{Underwater Communications}

The need for reliable underwater communications is necessary due to the emergence of a wide range of underwater sensor network applications. Applications of underwater communications fall into several domains [7]. Those domains include scientific applications for environment monitoring such as observing the geological processes on 
the ocean floor, water characteristics, marine life, deep sea archaeology, environmental studies, pollution monitoring, climate change and global warming. Other applications fall into the industrial domain that are related to oil, mineral extraction, underwater pipelines and commercial fisheries. Such applications involve control and monitoring through a network of actuators and sensors. There are also defense applications for underwater sensor networks that involve securing and monitoring port facilities and surveillance of coastal areas.

In general, there are several differences between space or terrestrial communications and underwater communications. The characteristics of the underwater environment determines the propagation of acoustic, electromagnetic or optical waves. These different characteristics have led to the need of new algorithms and protocols that would adapt to meet the requirements for reliable communications.

Several media have been considered for underwater communications. Fiber optics would be the best medium to consider for a high data rate. However, it is not always the case that a cable connection is an option. For instance, it is impractical and inadequate for underwater vehicles to use cables for communications. Another medium would be RF, which is used the most in terrestrial and space communications. However, $\mathrm{RF}[8,9]$ is only practical for a dozen of meters due to the conductivity nature of sea water, which affects the propagation of electromagnetic waves. Moreover, it requires large and powerful transmitters [10] to establish RF communications underwater. Similarly, optical waves for underwater communications have a high data rate, but again due to the nature of the water the optical waves signals are rapidly absorbed. In addition to that, the optical scattering is significant. Finally, the acoustic waves have low absorption losses in comparison to the aforementioned communications media especially at low frequencies. Each of the aforementioned physical medium has its advantages and disadvantages. However, acoustic waves effective range is in kilometres while electromagnetic waves effective range is around ten meters and optical waves is between 10 and 100 meters [11]. The main reason for using acoustic waves is the fact that they enable long range communications. 
On the other hand, acoustic wave communication faces physical challenges that radio communications does not [12]. Firstly, there is the low propagation speed, that is five orders of magnitude lower than RF. Secondly, there is significant reverberation due to multi-path propagation. Moreover, the available bandwidth is relatively small. Multipath propagation due to refraction and reflection affects the performance drastically. These effects can be mitigated by applying different techniques. These techniques are addressed in details in the upcoming chapters.

We define the concept of software-defined modem or software modem as a modem that consists of a Software-Defined Radio (SDR) peripheral connected to a host computer that utilizes it with a software to transmit and receive data. A SDR is a system where the components such as mixers, filters, amplifiers (de)modulators are implemented as software component on a computer or an embedded system rather than being implemented in hardware. The control of such a system is done by means of software that provide reconfigurability. This reconfigurability is applicable in various layers of the network [13]. The reconfigurability of SDR is useful for the challenges that an underwater communications channel present. Each underwater environment possesses its own characteristics that affect the performance of communications in various ways [14]. It is important to study different environments to understand their properties and effects on different modulation schemes and see what works best for each. 


\subsection{Statement of the Problem}

Underwater acoustic communications faces lots of complexities. They include noise, transmission loss due to absorption and geometric spreading, multi-path propagation and doppler spread.

The degree of absorption, in which acoustic energy is converted to heat in water, is relative to the chemical composition of water. Noise underwater is classified into two types, ambient and site-specific noise. Multi-path propagation can cause distortion to the transmitted signal due to arrival of several components at the receiver from different paths. Doppler spread also contributes in the signal distortion along with multi-path. These complexities cause high distortion to the transmitted signal. If these issues are not handled well it may not be possible to efficiently demodulate the signal. Arctic-like environments usually have an ice cover and an upward refracting sound speed profile. The impact of these two Arctic characteristics on different modulation techniques is significant. Different environment conditions may require different configuration of the modem to achieve a more reliable communication. In this thesis we took the task of finding what modulation scheme or transmission technique performs best in such conditions.

\subsection{Contributions}

The main contribution of this thesis is a framework for simulating an environment with an ice cover and multi-path propagation mimicking the underwater Arctic conditions, along with the study of their impact on the performance of several modulation schemes and transmission techniques. More specifically we have done the following:

- Hydroacoustic software modems are designed and developed within the GNU Radio environment. The modems support several types of modulation: Binary Phase-Shift Keying (BPSK), Frequency-Shift Keying (FSK), Frequency Hopping Continuous Phase FSK (FH-CPFSK) and Orthogonal Frequency Division Multiplexing (OFDM). For each modem, the symbol time is made adjustible, to mitigate Inter-Symbol Interference (ISI). The modems produced are a foundation for analyzing different underwater environments. 
- The approach taken is to design and test modulation schemes and transmission techniques while producing impulse responses that mimic the Arctic environment. For the simulation, ice cover data from Maritime Way Scientific Ltd. (MWS) has been provided to us. The BELLHOP software[15], a beam tracer used to predict acoustic pressure fields in ocean environments, has been modified by MWS in order to mimic the ice cover effects [16]. Ray models are valid in situations where the wavelngth of sound is much smaller than the water column depth, batymetric features and internal features such as surface ducts [17]. We use the modified version to generate the impulse responses that mimic the effects of the underwater Arctic-like conditions. Next, we convolve the impulse responses with the signals produced using the modems designed using GNU Radio. Finally, we demodulate the signals.

- A performance evaluation is conducted using the metrics Bit Error Rate (BER) vs Energy per bit to Noise power spectral density ratio $\left(E_{b} / N_{0}\right)$ and Packet Error Rate (PER) vs Signal-to-Noise Ratio (SNR). The results of the simulation show that at a symbol time that is much greater than the delay spread, FSK and FHCPFSK are more efficient than BPSK and OFDM in Arctic-like conditions. The reason is that the ice cover scatters the signals and distorts their phase. When there is no ice cover, FH-CPFSK outperforms FSK. FH-CPFSK and BPSK outperform FSK at higher data rates (lower symbol times). However, when the symbol time is much greater than the delay spread, FSK outperforms all the other modulation schemes and techniques at higher SNRs. We also concluded that in the conditions we simulated, BPSK performs better than OFDM with $80 \%$ ice cover and in an environment where we used same symbol time but with a range of $10 \mathrm{~km}$. OFDM has shown to be sensitive to the ice cover in the conditions we simulated.

\subsection{Organization of Thesis}

The upcoming chapters are organized as follow. Chapter 2 gives background on underwater communications and software-defined radio. Chapter 3 discusses the work 
related to underwater acoustic modems. The acoustic modem designs in GNU Radio are presented in Chapter 4. The evaluation methodology, simulation environments and simulation results are discussed in Chapters 5 and 6. Finally, Chapter 7 summarizes the work, gives the main conclusion and discusses future work. 


\section{Chapter 2}

\section{Background}

\subsection{Underwater Acoustics}

Acoustic waves are a mechanical phenomenon, unlike terrestrial or space communications that are based on an electromagnetic phenomenon. Underwater acoustic waves propagate according to different rules than those of terrestrial and space wireless communications. Underwater, acoustic waves support better range of communications than Radio Frequency waves (RF) or optical waves. However, underwater acoustic communication channel is not free of impairements. It is considered as one of the most challenging form of communications. It is characterized as a multi-path fading channel. It has been shown that most of the underwater channels are severely band limited with signal distortion ranging from benign distortion to extreme ones depending on the link configuration [11]. This distortion is due to time-varying multi path propagation $[18]$.

Underwater acoustic waves are subject to four main impairments [19]. The first impairment is absorption. It is an energy loss due to the conversion of mechanical energy to heat in water. Absorption causes transmission loss in which the signal intensity is weakened over distance. However, the effect of underwater absorption on acoustic waves is relatively low with respect to what it has on RF and optical waves. The degree of absorption is related to the chemical composition of the water. It is also frequency-dependent [11], therefore setting a limit to the available bandwidth [20].

The second type of impairment is geometrical spreading. As sound waves move further from their source, the area that the sound energy covers becomes larger and therefore the intensity of the sound waves decrease. 
The third type of impairment is noise. Noise is classified into two types: ambient and site-specific. Examples of ambient noise are turbulence, seismic shipping, waves and thermal noise. Thermal noise is generated by the random motion of water molecules and dominates at frequencies greater than about $100 \mathrm{KHz}$ [21]. While site-specific noise is produced by sea creatures and ice cracking.

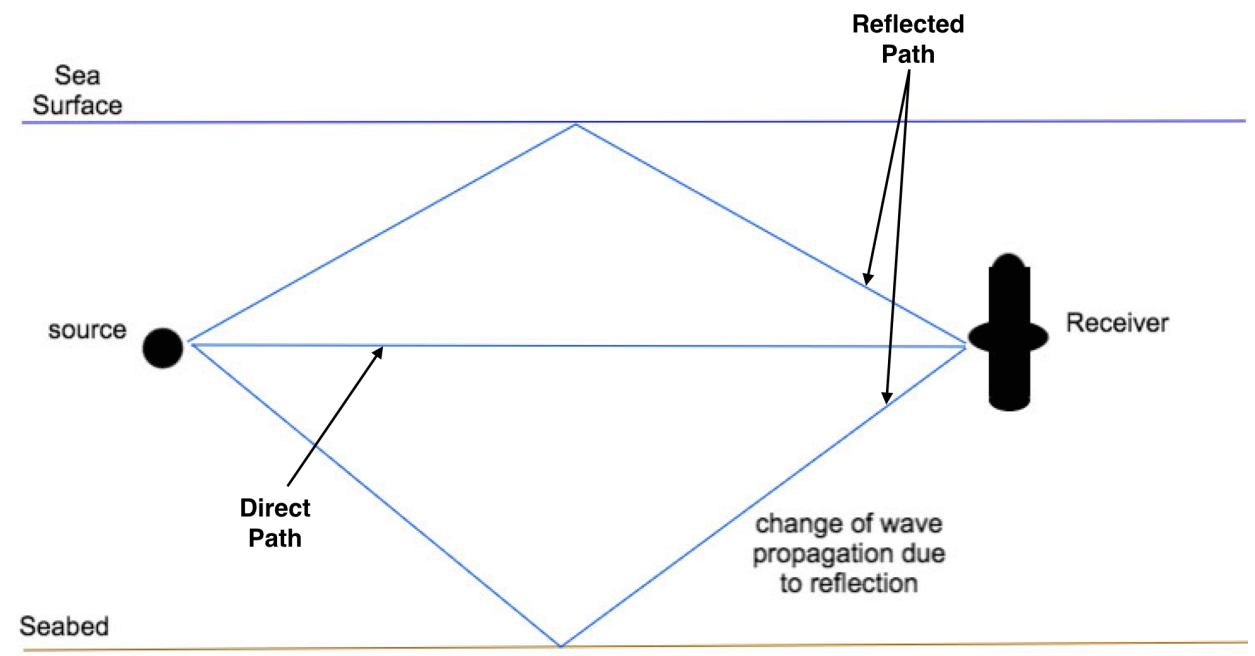

Figure 2: Example of reflected and direct paths. 
The fourth kind of impairment is the multi-path propagation of sound waves caused by refraction and reflection. Multi-path propagation is a phenomenon in which a signal reaches a receiver through more than one path. It causes both destructive and constructive interference as well as phase shifting. Multi-path propagation depends on the communications direction. If the direction of communications is vertical, the channel experience less reverberations than for a horizontal channel [22]. Refraction and reflection are determined by the environment boundaries, sound speed profile and the transmitter and receiver positions. The sound speed profile defines the speed of sound as a function of underwater depth. The sound speed profile depends on underwaterwater temperature, salinity and pressure [11, 20].

The two phenomena of refraction and reflection are depicted in Figures 2 and 3. Figure 2 shows a direct path, a seabed-reflected path and a sea surface-reflected path in an iso velocity (constant sound speed) environment. Reflected paths tend to be weaker since they travel longer distances and are absorbed by the reflectors, seabed and ice cover. Refraction is due to the change of the underwater sound speed. Figure 3 shows how sound waves refract as they go from one medium with sound speed $C_{1}$ to another with sound speed $C_{2}$. In this example, $C_{1}$ is larger than $C_{2}$. Refraction occurs between the sea surface and seabed. Refraction follows Snell's law. Changes in the direction of wave propagation due to refraction is determined by the sound speed gradient, that is the rate of change in sound speed versus the depth of water. The larger the sound speed gradient, the steeper is the change of direction. 


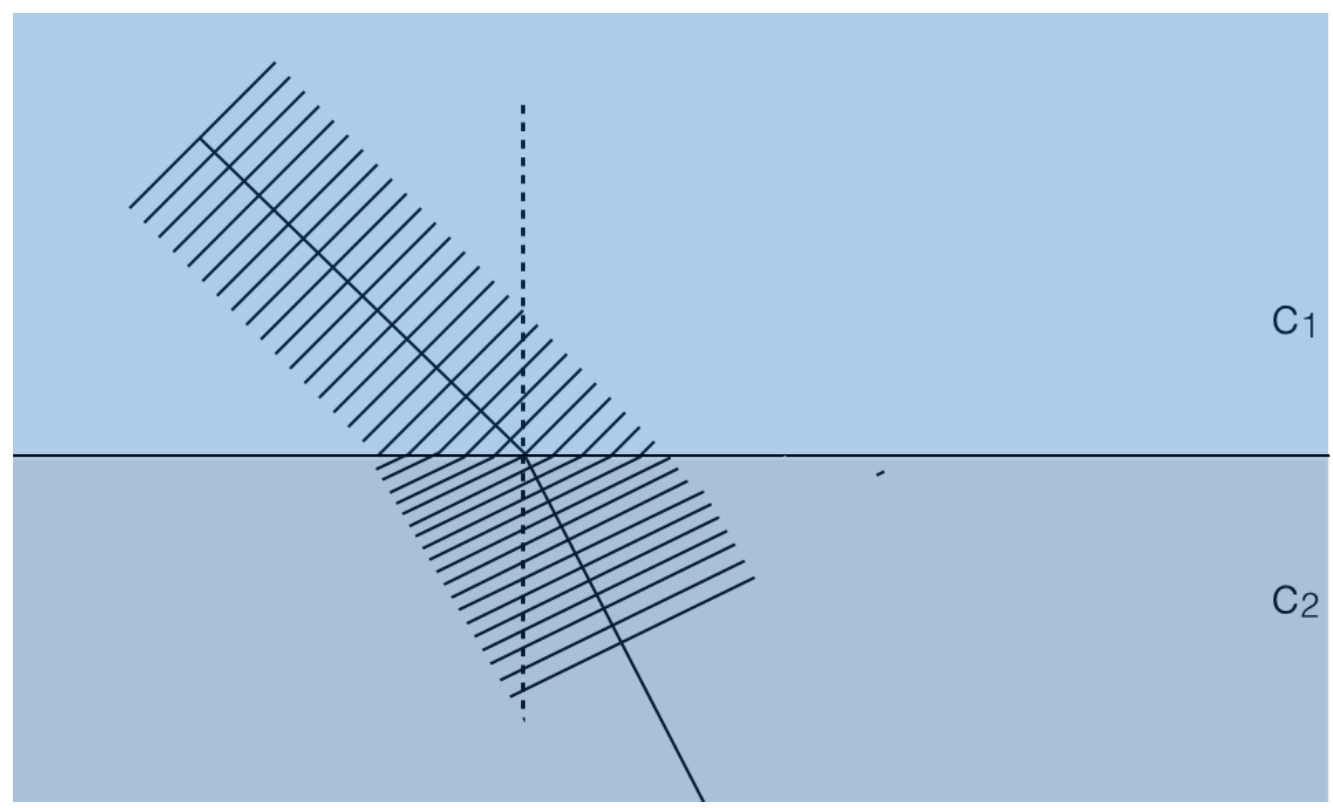

Figure 3: Refraction $\left(C_{1}\right.$ is larger than $\left.C_{2}\right)$.

Figure 4 shows a sound speed profile where the rate of change in speed is high in shallow water. The speed starts at $1550 \mathrm{~m} / \mathrm{s}$ at the surface. It drops to $1500 \mathrm{~m} / \mathrm{s}$ at around 1200 meters. The speed then gradually increases, where it reaches 1550 $\mathrm{m} / \mathrm{s}$ at a depth of 5000 meters. Figure 5 shows the eigenray plot produced using the BELLHOP software [15] in an environment where the sound speed profile is the one shown in figure 4. Each eigenray represents a propagation path from the transmitter to the receiver. 


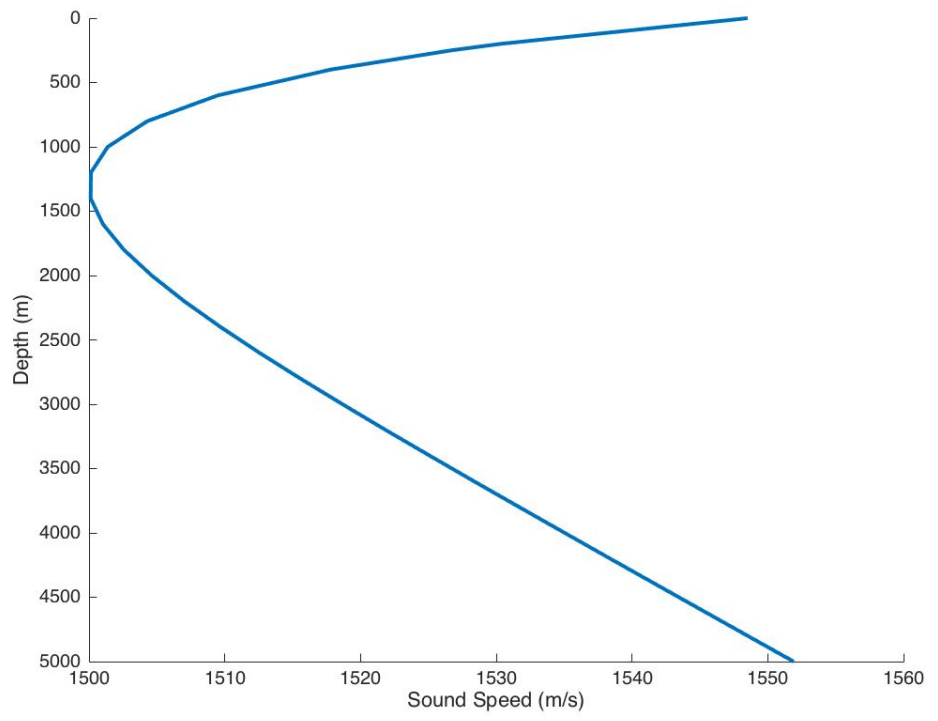

Figure 4: An example of a sound speed profile.

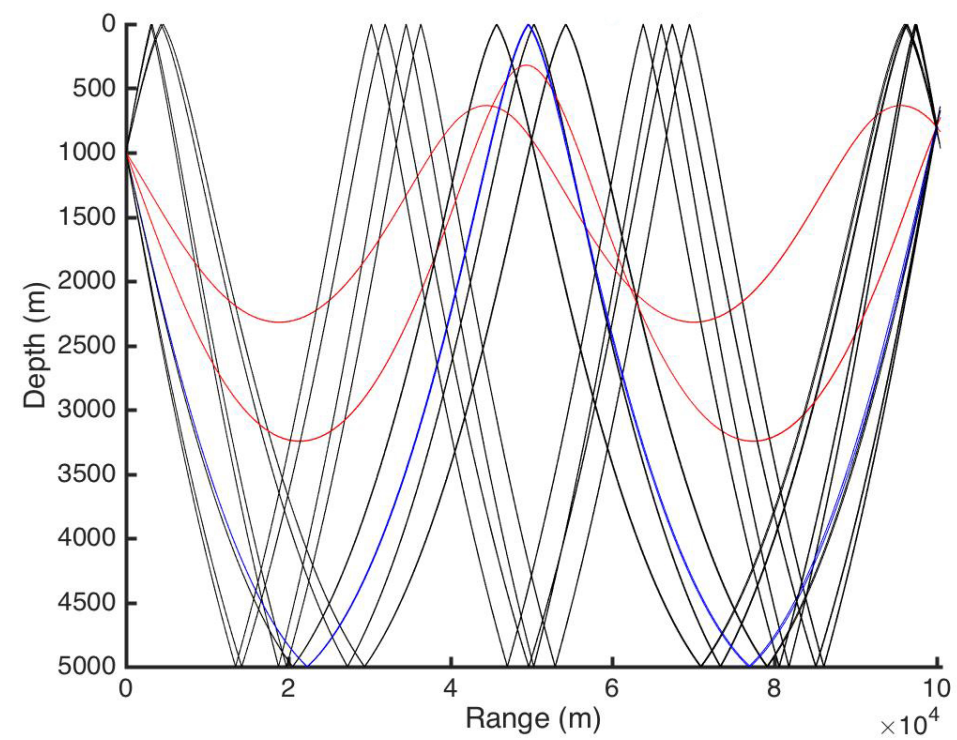

Figure 5: Eigenrays generated in an environment with refracted paths (in red) and reflected paths (in black). 
A signal arriving at the receiver from several paths due to multi-path propagation causes distortion. Multi-path introduces Inter-Symbol Interference where the signal that represents a data symbol interferes with adjacent symbols [9]. Figure 6 shows how a transmitted signal is received in the case of a single path and in the case of a multipath propagation. The Power Delay Profile (PDP) gives the intensity of a signal as a function of time. ISI impedes the efficient demodulation of the original signal. To mitigate the effect of ISI, certain modulation techniques can be applied.

Another underwater communications impairment is Doppler spread, in which the frequency dispersion of the signal occurs due to the interaction with moving objects. Examples of moving reflectors are sea surface and mobile transmitters and receivers. The frequency variation at a given time is called spectral shift. The Doppler spread is the standard deviation of multiple spectral shifts. The inverse of the Doppler spread gives an approximation of the coherence time of the channel, which is the time duration over which the impulse response of the channel is constant. The inverse of the Root Mean Square (RMS) delay spread, is the standard deviation of the PDP. It is proportional to the coherence bandwidth of the channel.
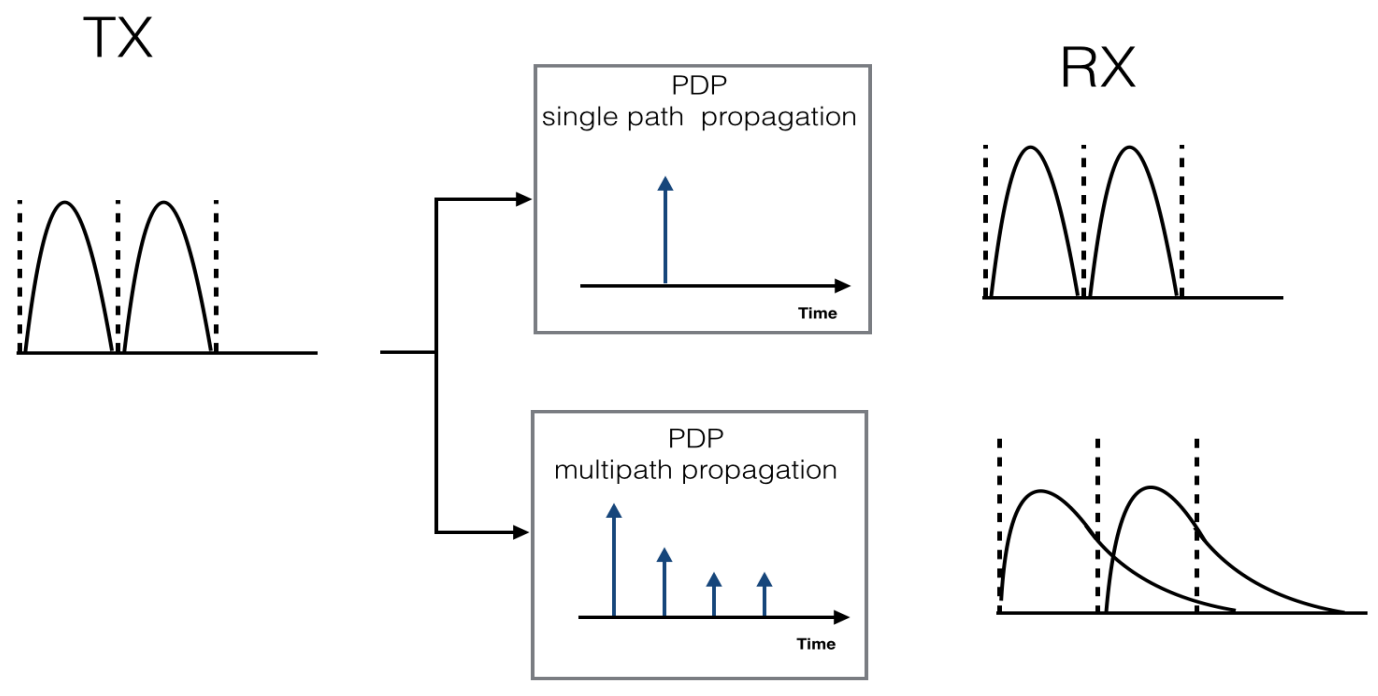

Figure 6: Single path vs multipath propagation between a transmitter (TX) and a receiver $(\mathrm{RX})$. 


\subsection{Software-Defined Communication}

Section 2.2.1 talks about Software-Defined Radio (SDR) or software-defined communication system in general and their capabilities over traditional radio and communication systems. Section 2.2.2 introduces GNU Radio, a software development toolkit used to develop the software-defined hydro-acoustic modems.

\subsubsection{Software-Defined Communication Systems}

SDR or Software-Defined Acoustic Communication (SDAC) in our case, is a communications system where filters,(de)modulators, detectors are implemented in software rather than in hardware. A software-defined modem consist of a SDR peripheral and a software on a computer or an embedded system. The peripheral is a bridge between the analog world (transducers) and digital world. It is connected to a host computer that controls it with a software to transmit and receive data.

$\mathrm{SDR}$ in general is unique due to its ability to process the signals in software. This is done using Digital Signal Processing (DSP). To make use of SDR, the analog signal must be converted to and from the digital domain. This transformation is done using digital-to-analog converter ( and analog-to-digital converter. Once the signal is in the digital domain, processing is done using DSP algorithms.

SDAC provides reconfigurability. It is capable of implementing digital filters that might be challenging in the analog domain. These filters can be tailored to adapt around interferers and compensate for transmission path distortion. Both features are of great difficulty to accomplish with analog filters [23].

In general, reconfigurability is defined as the ability to change the configuration of a system, such that it modifies its behaviour or the way it operates. Reconfigurability can be static or dynamic. With static reconfigurability the system configuration can be changed on startup and not during runtime. In contrast, with dynamic configuration the system can be changed during runtime. In the context of acoustic communications, reconfigurability applies to the behaviour of the network, network protocols, 
signal processing algorithms [13]. Reconfigurability is useful for the challenges that an underwater communication channel presents, since different underwater environments may require different filter adjustments, equalizers or modulation techniques. In the case of SDAC, the transmission is determined through software. This feature gives the capability of changing modes through software. This is done by manual configuration, through a software update or the use of signal analysis done by the system to determine how to change the current mode, an approach which is known as cognitive system. In the cognitive approach, a system is aware of its internal state and environment. It makes decisions about its operations by mapping that information against predefined objectives [24]. In this project, we use SDAC to create a software modem that can use different modulations. This enables evaluations of different modulations in certain underwater environments. The reconfigurability here is done manually.

SDAC has the capability of multi-mode, multi-rate, multi-carrier and variable bandwidth operations. Multi-carrier is as the name suggests, the ability to simultaneously operate on more than one frequency. Multi-mode is the ability to process several different kinds modulation schemes and transmission techniques such as AmplitudeShift Keying (ASK), FSK, Gaussian Minimum-Shift Keying (GMSK), either simultaneously or sequentially. Multi-rate enables the processing of different parts of the signal using different data rates or processing using different modes where each mode operates on a different data rate. The variable bandwidth feature is the capability of determining the bandwidth by means of digital filters.

\subsubsection{GNU Radio}

GNU Radio [25] is a software framework that provides signal processing blocks and a model to implement software radios. Basic concepts of GNU Radio are flow graphs and blocks. A flow graph consists of connected nodes or blocks through which data flows. The blocks are connected by arrows. Each arrow denotes a data flow direction. The actual signal processing is done in the blocks. The developer can use the blocks provided by GNU Radio to create flow graphs that make up a software radio or 
more generally a software communication system. Moreover, the developer can create his/her own blocks using either Python or $\mathrm{C}++$, to be used along with the blocks provided.

One can specify variables to be used among several blocks. Different GNU Radio blocks may support different data types. A block may also take values as arguments. Those values are used in the processing of the signal.

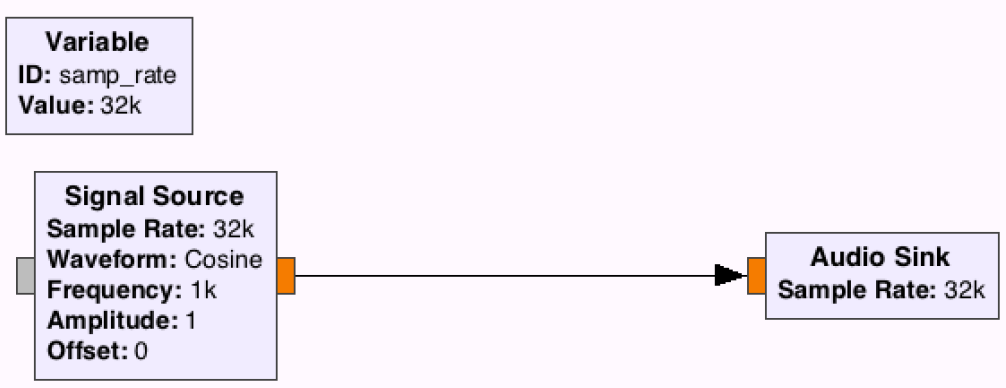

Figure 7: A GNU Radio flowgraph.

In Figure 7, one can see a signal source with a link to an audio sink. The link originates from a port in the signal source block and connects to another port in the audio sink block. The data types of the ports connected between the source block and receiver block must match. GNU Radio generates Python files which are the software modem. However, these files use GNU Radio libraries. It is also important to note that a flow graph as seen in Figure 7 can be hierarchal, in which a block inside of the flow graph can actually consist of another flow graph. GNU Radio is a softwaredefined development framework, nevertheless it can be used to create simulations. For instance, this is done by using channel blocks that act as filters on the signals to simulate the channels. GNU Radio provides a wide variety of channel simulation blocks. 


\section{Chapter 3}

\section{Related Work}

\subsection{Underwater Communication Modems}

We review the literature of underwater acoustic modems, focusing on the SDR approach and physical layer techniques addressing communication impairement. Furthermore, both the data link and network layers [26] are also considered in some of the papers reviewed.

Demirrors et al. [27] propose a software defined real-time reconfigurable modem. It is implemented using GNU Radio and MATLAB ${ }^{\mathrm{TM}}$. A high-level hardware architecture is presented. A complete overview of the design considerations for physical, data link and network layers of the protocol stack is provided. At the physical layer two signalling techniques are considered, zero-padded OFDM (ZP-OFDM) and Direct Sequence Spread Spectrum (DSSS). Both aim at maximizing the channel utilization. The ZP-OFDM implements an error correction scheme. The zero padding works as a guard interval alternative to cyclic prefix. Binary PSK (BPSK) and Quadrature PSK (QPSK) are both considered in OFDM. A guard interval is added between each OFDM symbol to avoid interference. A Pseudorandom Noise (PN) is sent at the beginning of each OFDM packet for frame detection and coarse synchronization. Null subcarriers are used for Doppler spread estimation and compensation. Pilot subcarrier are used for channel estimation and fine synchronization in the frequency domain. The second technique used is DSSS. To mitigate ISI, the product between the code length, of the binary code-wave form, and waveform duration is larger than the multipath spread. Two transmitter designs are considered. The first one is similar to OFDM. It uses PN for frame detection, synchronization and channel estimation at the receiver side. The second is an all-one sequence of unmodulated bits used to achieve 
frame detection and coarse synchronization. The adaptation is achieved using a feedback link based on binary chirp spread spectrum. The receiver communicates the decisions made to the transmitter according to the signal-to-interference-plus-noiseratio (SINR) . The modem is capable of switching between the two techniques based on multiplexer-based adaptation. Real-time code waveform adaptation in the DSSS link to maximize the SINR. At the data link layer, a Medium Access Control (MAC) protocol is implemented. The user sends and waits for acknowledgement. There is a timeout for retransmission. At the network layer, an adaptation of the IPv4 and IPv6 protocols is implemented. It interfaces the traditional IP network layer with the MAC layer of the prototype.

Wu et al. [28] have designed an adaptive acoustic modem for underwater sensor networks. Adaptation here is on both the modulation scheme and data rate. When the channel multipath increases, the modem switches to a lower data rate to mitigate ISI. The structure of the adaptive modem is described. It has a controller module that manages the modem. The two modulation schemes considered are DSSS and FSK, with non-coherent energy detection. Channel estimation is done for Doppler shift, channel path gain and SNR. The channel estimation is accomplished using a chirp signal. The receiver communicates the channel measurements to the transmitter. The estimation of the Doppler shift is defined as the product

$$
\hat{f}=\delta f
$$

where $f$ is the frequency of the carrier $(\mathrm{Hz})$ and the ratio delta is defined as

$$
\delta=\frac{T_{r p}}{T_{t p}}-1 .
$$

The variables $T_{r p}$ and $T_{t p}$ are the durations of the transmitted and the received packets. The multipath power delay profile is calculated using a chirp. They perform symbol synchronization using correlation with a known preamble.

A software modem for underwater acoustic communications is proposed by Borowski and Duchamp [29] with both physical and datalink layers written in Java. The modulation scheme used is Frequency-Division Multeplixing (FDM) combined with binary 
and 4-FSK. The transmitter sends one Linear Frequency Modulated (LFM) chirp per packet. This chirp is used at the receiver side for packet synchronization as well as channel estimation with the option of applying the impulse response estimate to the channel equalization. This is done by applying the inverse of the channel impulse response to the modulated data signal. The user edits the modem configuration through a text file. A guard time is inserted after the LFM chirp. The demodulation is done by applying the Hilbert transform to create an analytic signal, then computes its absolute value. The demodulator then compares the envelopes and chooses the symbol that corresponds to the tone with the larger amplitude.

SeaModem is an underwater acoustic modem for shallow water communications by Giaani et al. [30]. It operates in the frequency range 25 to $35 \mathrm{kHz}$. It is implemented on a DSP board. It comprises reconfigurable Multiple FSK (MFSK) parameters, which include choosing 2, 4 or 8 FSK modulation, error detection and error correction algorithms. Benson et al. [31] present a Field-Programmable Gate Array (FPGA) modem with a home made transducer using a piezoceramic material. FSK modulation is used.

A performance comparison between MFSK, Frequency-Repetition Spread Spectrum (FRSS) and Direct-Sequence Spread Spectrum (DSSS) in the underwater channel has been conducted, by Walree et al. [32], has shown that MFSK gives a better overall performance at high SNR while FRSS gives a better performance at low SNR. FRSS is similar to DSSS except that FRSS chops the symbols in frequency using $\mathrm{k}$ subbands while DSSS chops them in time using $\mathrm{k}$ chips. A channel simulator driven by an in-situ impulse response from seven geographical areas in $4-8 \mathrm{kHz}$ band is applied to distort the signal. All of the schemes are equipped with m-sequence preamble. A filter matched to a bank of Doppler shifted preamble replicas is used for detection and synchronization. The estimated Doppler shift is removed by resampling. The authors conclude that there is no scheme that performs best in all environments. 
The work done by Dol et al. [33] reviews briefly the literature and NILUS approach. The authors highlights the need for an adaptive platform with capability of changing its characteristics to suit the requirements of different environments and scenarios. The work describes relevant projects on reconfigurable modems. Those modems include MIT Sea Grant Modem 2006-2008, aimed at building a hardware which is reconfigurable at the physical layer. The modem achieved a rate of $550 \mathrm{bps}$ using QPSK within a tank using $12 \mathrm{kHz}$ carrier.

The second modem is the MIT CSAIL's AquaNodes which has a fixed physical layer and reconfigurable network protocols. It achieves 330 to 400 bps using FSK and a $30 \mathrm{kHz}$ carrier.

The third modem is the University of Connecticut's OFDM modem. It is based on MIMO-OFDM signal processing, equipped with two transmitters and 16-QAM modulation. The modem is capable of transmitting $127.7 \mathrm{kbps}$ at $62.5 \mathrm{kHz}$ bandwidth.

The fourth modem is the UCLA's underwater acoustic networking platform. It is a reconfigurable at both the physical and network layers. It utilizes both GNU Radio and TinyOS for that purpose.

The paper also considers the Ecologic Wise modem series. They embed a networkdevelopment framework in a sandbox that supports NS2 simulation [34].

Subnero's modem supports reconfigurability at both physical and network layers. The Subnero modem would run a 500 bps command link along with 2 to $10 \mathrm{kbps}$ data link on a $27 \mathrm{kHz}$ carrier and $18 \mathrm{kHz}$ bandwidth.

The review also includes the Teledyne Benthos modems. They can be reconfigured through an application programming interface. It enables the bypass of the native networking protocols to command the physical and datalink layers. 
Finally, the NILUS software Modem is a framework consisting of physical, datalink and network layers using VAR-SOM-OM44 computing platform. The modulation scheme used is FRSS. The MAC protocol used is ALOHA with carrier sensing.

This concludes our review on the related work. One fact we observed is the absence of comparitive evaluation of modulation schemes and transmission techniques in Arctic-like conditions. The construction and evaluation of modems for Arctic-like conditions is the purpose of the upcoming chapters. 


\section{Chapter 4}

\section{Modem Design}

Modem design for mitigating the effects of ISI is discussed. Implementation using GNU Radio available Digital Signal Processing (DSP) blocks is presented. This includes several modulation techniques for both the transmitter and receiver.

\subsection{Introduction}

This chapter introduces the techniques and modem design used to address underwater channel impairements. A contribution of this work is the creation of a number of software-defined underwater acoustic modems. The emphasis is on modulation techniques used in underwater acoustic communications [11]. They consist of Frequency Hopping CPFSK (FH-CPFSK), multicarrier modulation Orthogonal Frequency-Division Multiplexing (OFDM) and single carrier modulation using the schemes Phase-Shift Keying (PSK) and Frequency-Shift Keying (FSK). In this thesis, we simulate those techniques in Arctic-like conditions, where there is an ice cover that scatters the signal as a result of the reflections and upward refractions due to the sound speed profile. 


\subsection{Single-Carrier Modulation}

We consider two modulation schemes for single-carrier modulation: PSK and FSK. Both are known to have good noise tolerance. In the following, we discuss PSK and FSK modulations in detail.

\subsubsection{Phase-Shift Keying Modulation}

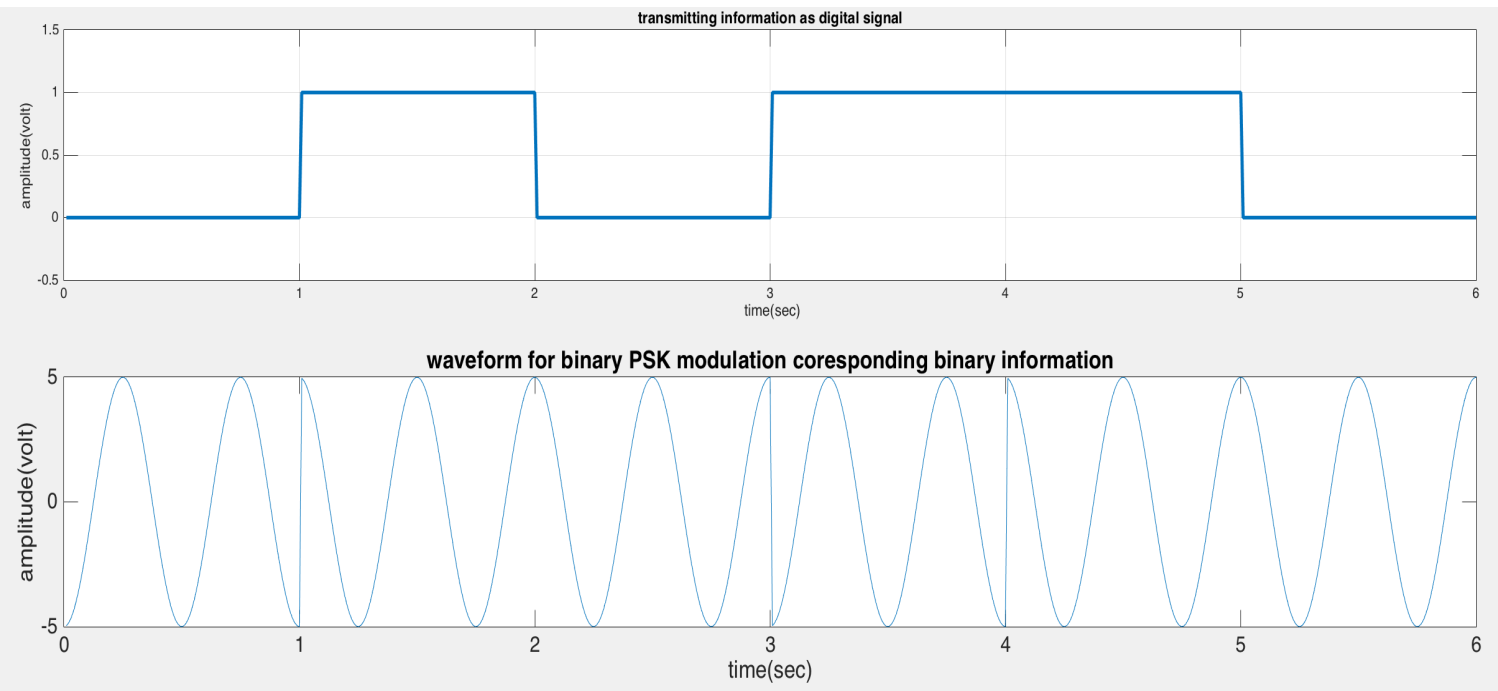

Figure 8: Time domain view of a DPSK signal.

PSK is a digital modulation scheme used for data communications. It is mostly used in terrestrial communications including wireless local area networks, Bluetooth and radio frequency identifications.

The conveying of information in PSK is accomplished by encoding data in the phase of the signal. This is done by assigning different phases to binary digit patterns. Each phase represents the same number of bits. With more phases longer patterns can represented. Higher data rates can be achieved. The demodulator determines the phases of signal symbols and maps them to bit patterns. Differential PSK (DPSK) uses the previous symbol as a phase reference fto determine the phase of the current symbol. A time domain DPSK signal waveform is shown in Figure 8. ${ }^{1}$ The figure also shows the mapping of bits to signals. The upper part shows the transmitted bit

\footnotetext{
${ }^{1}$ Generated using the code adapted from [35]
} 
sequence i.e., 010110. Each bit is mapped to a DPSK symbol. At the begining of each symbol, the signal makes a phase transition, in this example 0, 180, 0, 180180 and 0 degrees. In the lower part the amplitude (in volts) of the signal is shown as a function of time (in seconds).

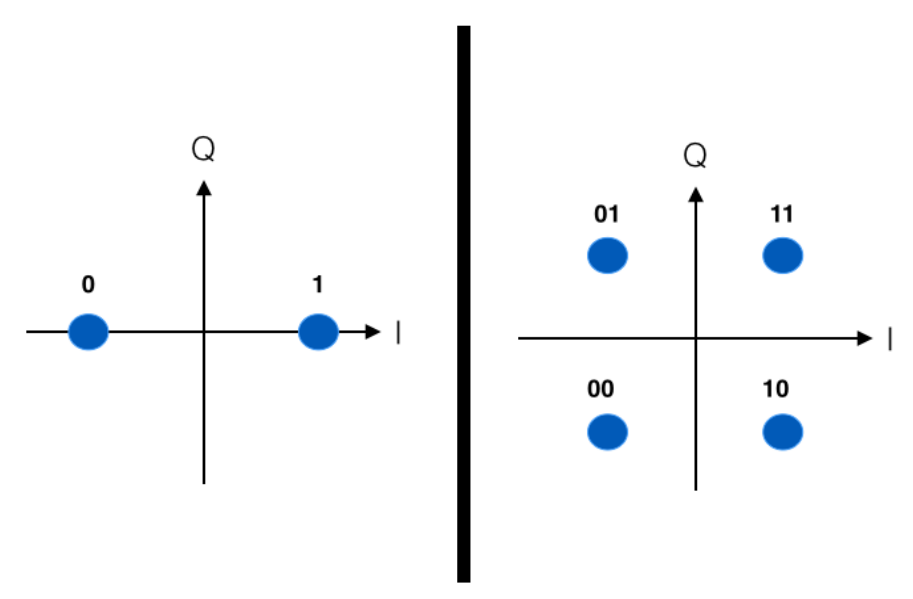

Figure 9: BPSK and QPSK constellations

Figure 9 shows the constellation diagram for both BPSK (on the left side) and QPSK (on the right side). A constellation diagram is another representation of the modulated signal. It shows the possible symbols that may be used in M-ary PSK modulation. The constellation points shown in blue in each of the BPSK and QPSK constellation diagrams represent a possible symbol mapping. The QPSK constellation diagram shown uses Gray coding, in which only one bit changes between two successive points. Note that it is possible to use Gray coding which also minimizes the bit error rate.

An M-ary PSK signal can be defined as a function of time $t$ by the equation

$$
s(t)=A \cos \left(2 \pi f t+\frac{2 \pi i}{m}\right)
$$

where $f$ is the carrier frequency $(\mathrm{Hz}), i=1 \ldots m$ and $A$ is the amplitude (volts). 


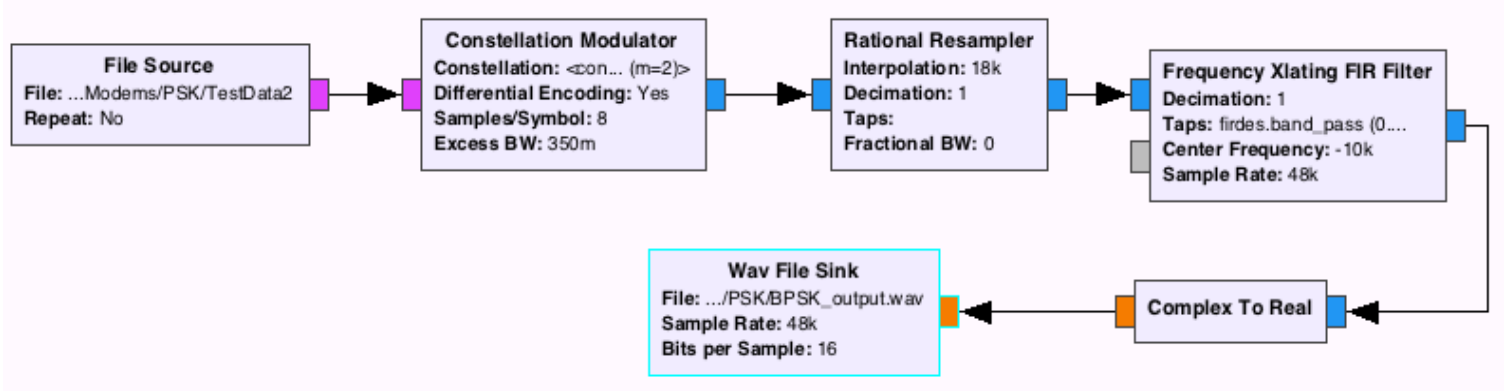

Figure 10: BPSK transmitter module.

In this section, we look at the modem design for PSK in GNU Radio. It consist of a transmitter and a receiver modules.. Figure 10 shows the transmitter module with a file source of data to be transmitted. The data goes to the constellation modulator. The constellation modulator takes as argument a constellation object that defines the constellation points and symbol mapping. The constellation modulator produces a baseband complex modulated signal with eight samples per symbol. It is filtered using a root raised cosine pulse shaping filter. The samples are then interpolated using the Rational Resampler block to meet the symbol time requirement. Next, we perform frequency translation using Frequency Xlating Finite Impulse Response (FIR) filter at the rate 48,000 samples/seconds. Finally, the signal is converted from complex to real valued. It is ready to be either saved in a .wav file or broadcasted using an audio sink block in GNU Radio.

Symbol time calculation is done as follow

$$
T_{s}=\frac{\text { sps } \cdot \text { Interpolation }}{\text { SamplingRate }}
$$

where sps is the samples per symbol. Interpolation denotes the interpolation factor [36].

In order to have reliable underwater communications using single carrier modulation, we must either make sure the symbol duration $T_{s}$ is much greater than the delay spread, or apply equalization to suppress the interference caused by the multipath propagation. However, it is a challenge to apply such techniques in a fast-varying 
channel [11]. In the following, we do not use equalization. We set the symbol time such that it is much greater than the delay spread.

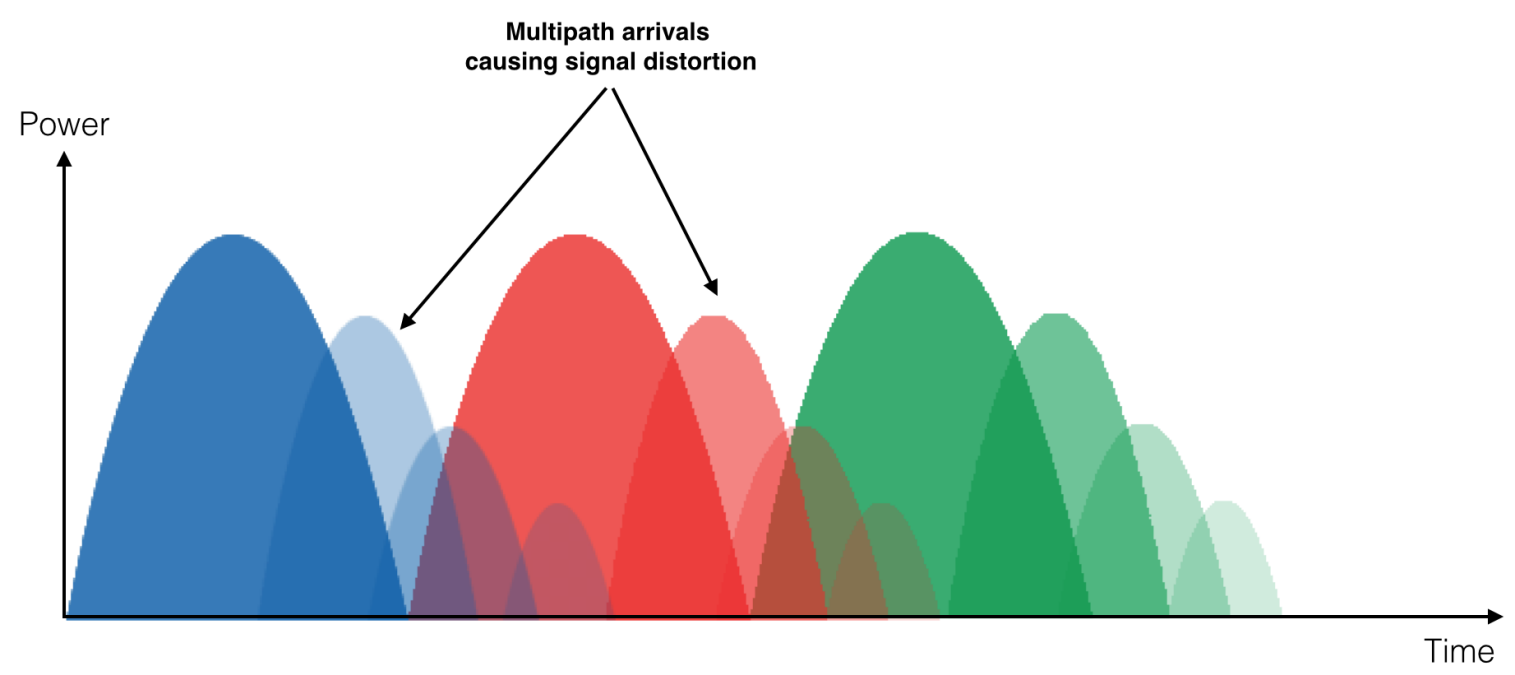

(a) $T_{s}$ smaller than or equal to the delay spread.

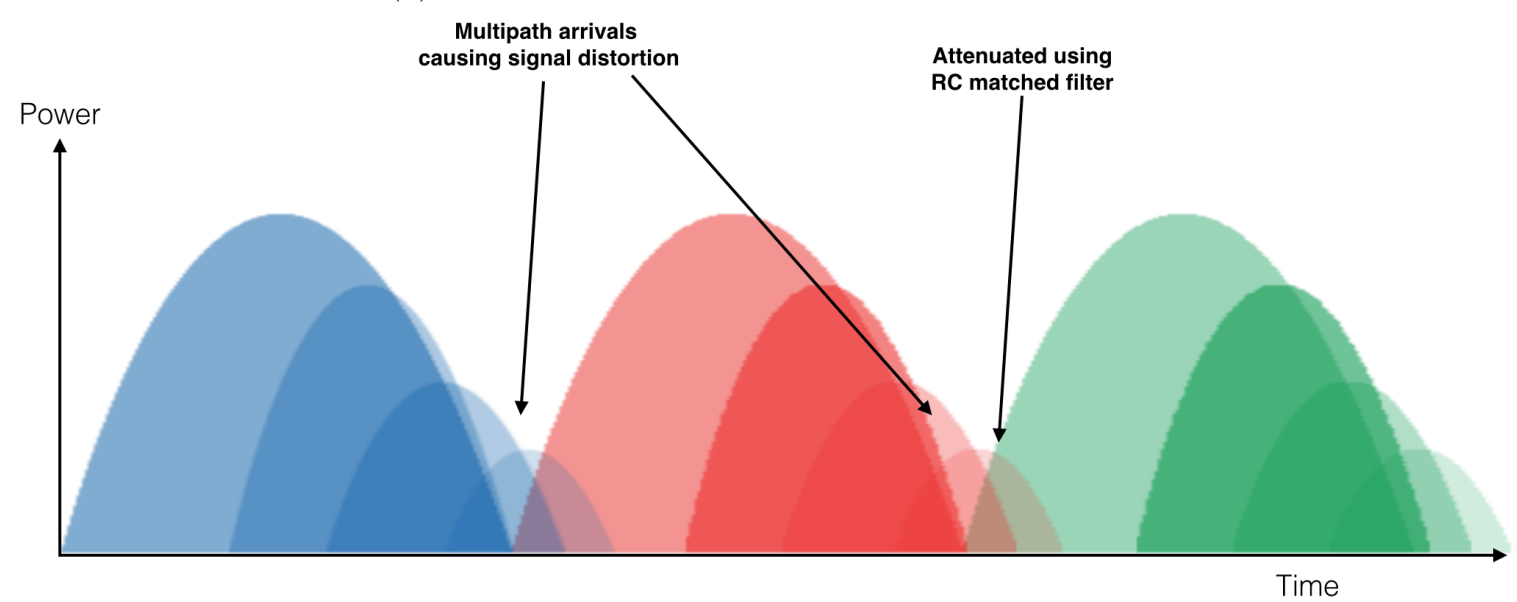

(b) $T_{s}$ larger than delay spread.

Figure 11: Mitigating channel effects with PSK single-carrier modulation. 

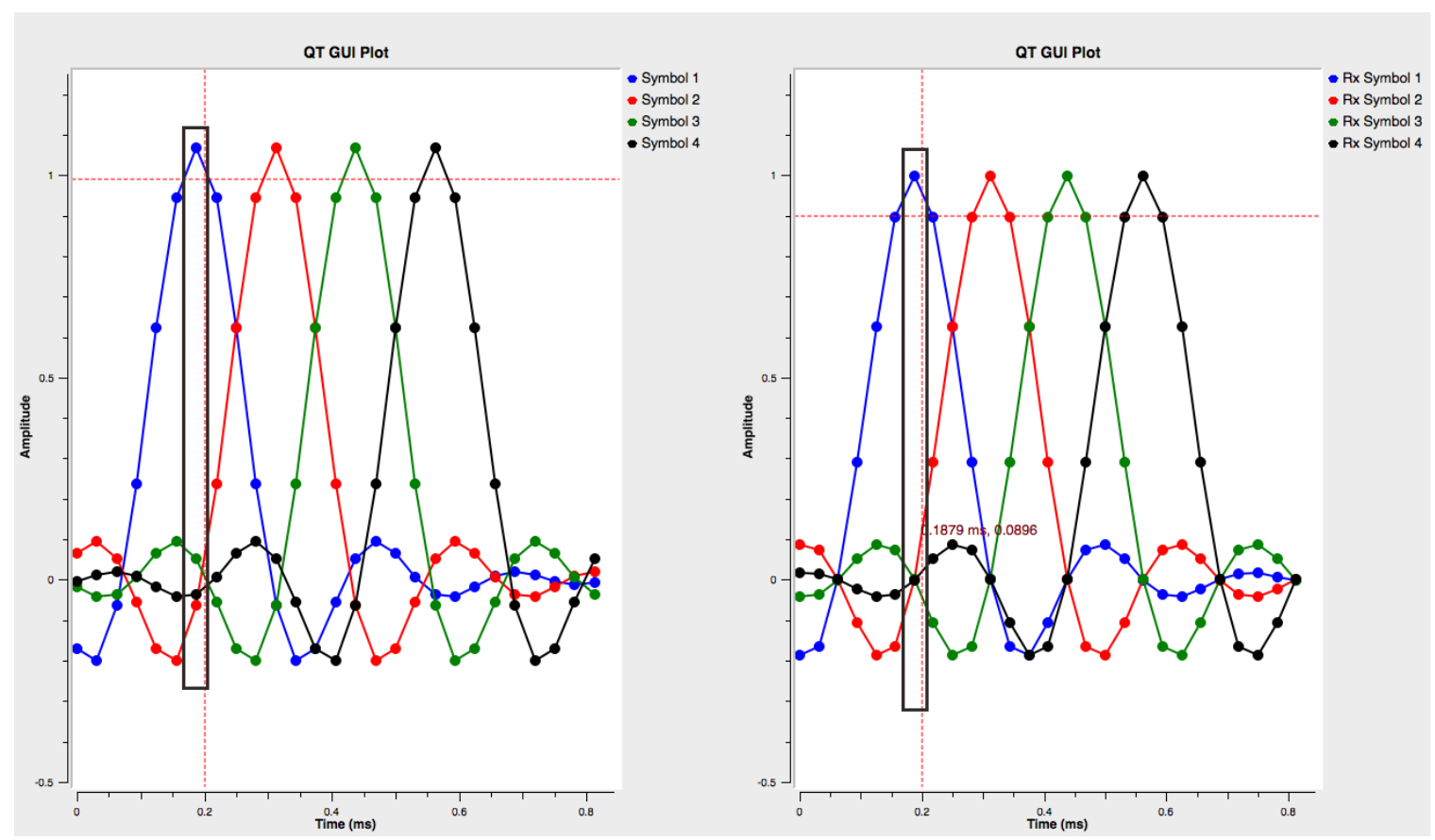

Figure 12: BPSK sampling point with raised cosine filter.

Adjusting the symbol time alone is not sufficient. To achieve better performance, we apply a matched filter using a raised cosine filter to suppress the interference as shown in Figure 11. Each cluster of peaks represents a symbol. The members in each cluster correspond to the multipath arrivals. In the upper part, we have the original signal where the symbol time is less than or equal to the delay spread. There is no match filtering. There is a significant amount of intercluster overlap. In the lower part, the symbol time is larger than the delay spread and raised cosine filtering is applied. The intercluster overlap is significantly lower. A matched filter is achieved by correlating a root raised cosine filter with the received signal at the receiver.

The technique of applying a matched filter attenuates the magnitude at the beginning and end portions of the symbol period. At the transmitter, the root raised cosine filter serves the purpose of generating a signal with no symbol overlapping. At the receiver, the root raised cosine filter filters out the reflections caused by the multipath arrivals, therefore minimizing the ISI. Figure 12 shows the importance of a matched filter when sampling the signal. The left side of the figure shows a signal with no 
matched filter. The samples taken from the left side graph contain energy from the neighbouring symbols even at the ideal sampling point therefore distorting the signal. However, at the ideal sampling point of the right side graph, there is zero energy from the neighbouring symbols. In a real multipath environment, we get some energy from neighbouring signals. However, it is minimized with a matched filter. 


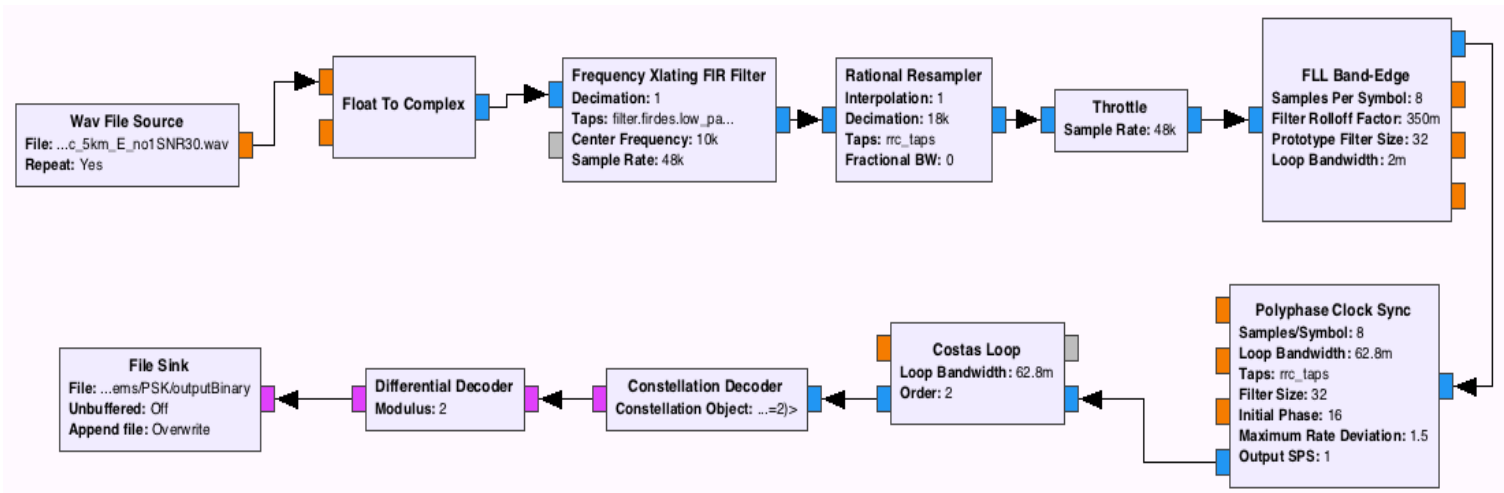

Figure 13: BPSK receiver module.

The receiver module depicted in Figure 13 is fed with a signal from an audio source. The signal is converted to complex. The Frequency Xlating FIR Filter block translate the frequency back to baseband signal. A Rational Resampler performs decimation. The signal then passes through a FLL Band-Edge filter which does coarse frequency tracking to compensate for Doppler shifts. As shown in Figure 14, in (a) there is no frequency offset, in (b) there is a frequency offset and finally in (c) the received frequency offset is corrected using a Frequency Locked Loop (FLL) Band-Edge filter. According to the author of this block, it works as the following:

- It creates a filter for each edge of the transmitted signal in frequency, given the excess bandwidth of the pulse shaping filter.

- The coarse frequency offset is relative to the difference in the amount of energy between the two filters. 


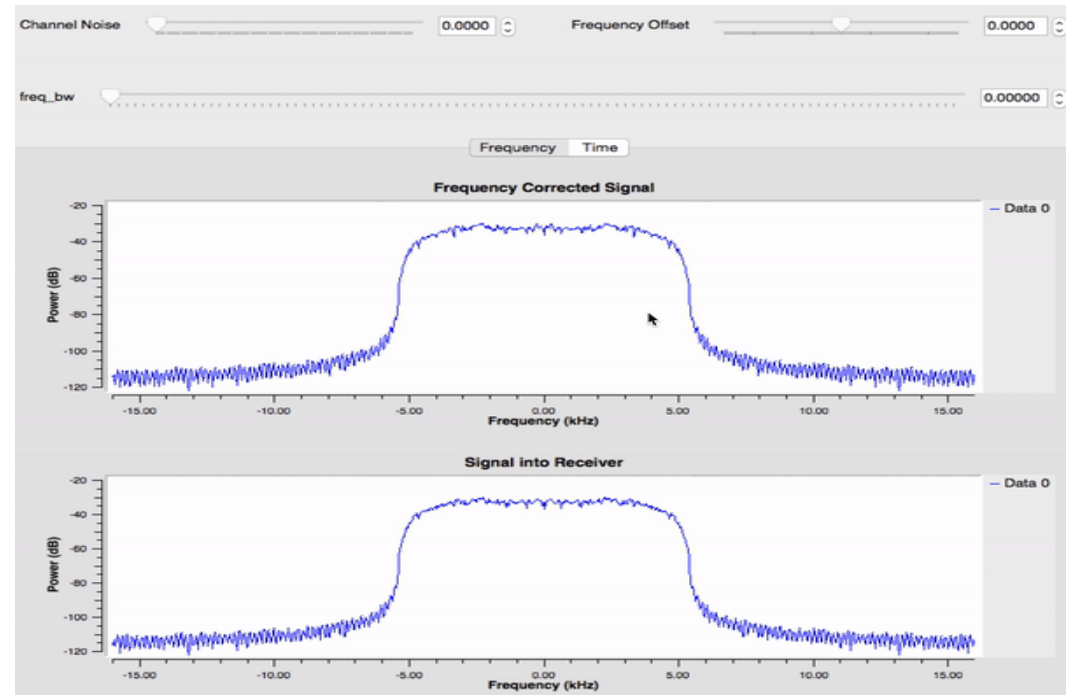

(a) No frequenct offset

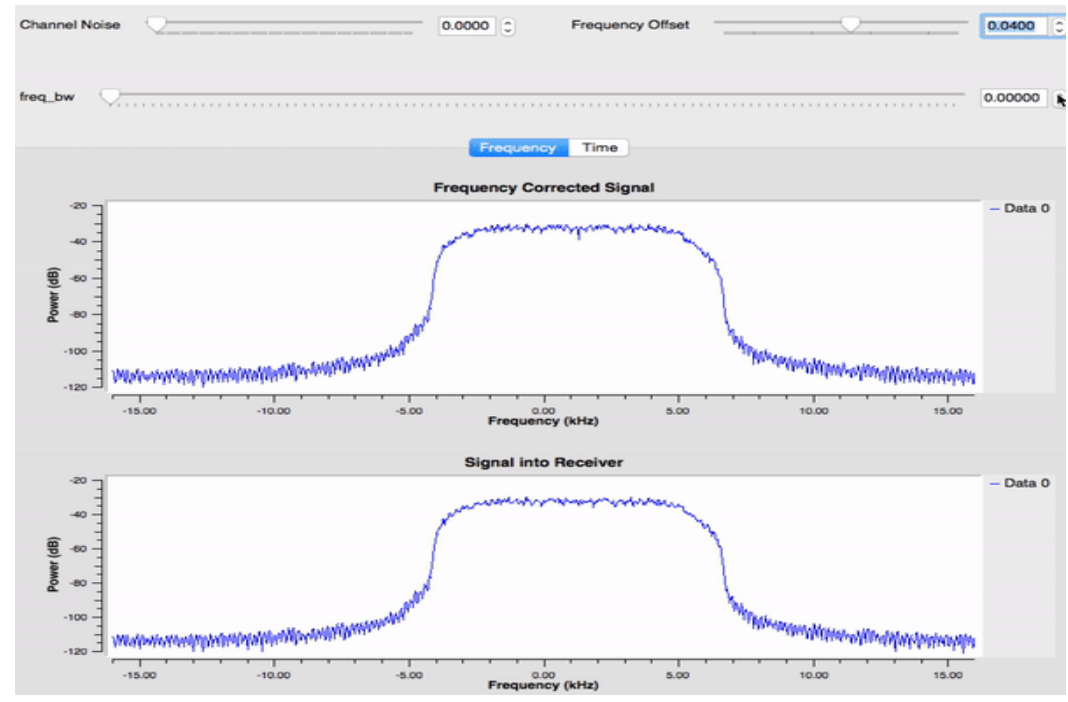

(b) Introducing frequency offset

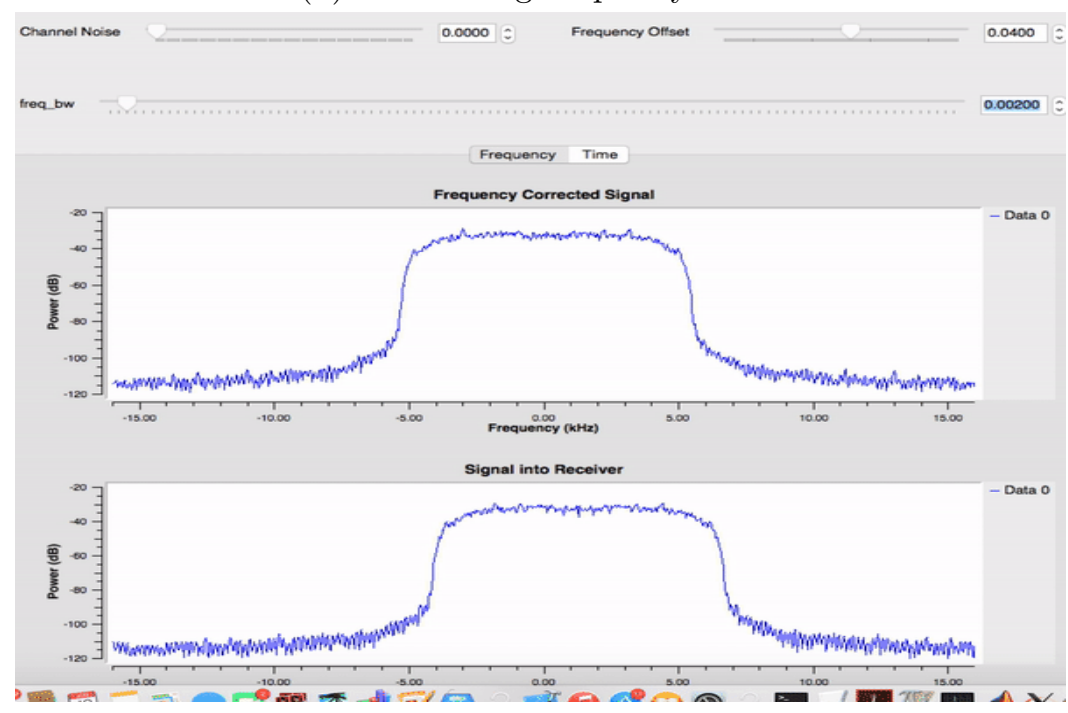

(c) FLL Band-Edge correcting for the frequency offset

Figure 14: FLL Band-Edge. 
After coarse frequency correction, the signal passes through a PolyPhase Clock Synchronizer block for timing recovery, where the timing offset between the receiver sampling clock and optimal sampling position is removed. The polyphase finds the best sampling point by creating a bank of differential filters similar to what is shown in Figure 15 with different phase offsets. The filter that has an output of zero or close to zero has the phase offset to use in the matched filter. After applying the matched filter, we sample at the point where the differential filter produces a zero. The PolyPhase Clock synchronizer applies the matched filter and downsamples the signal to one sample per symbol.

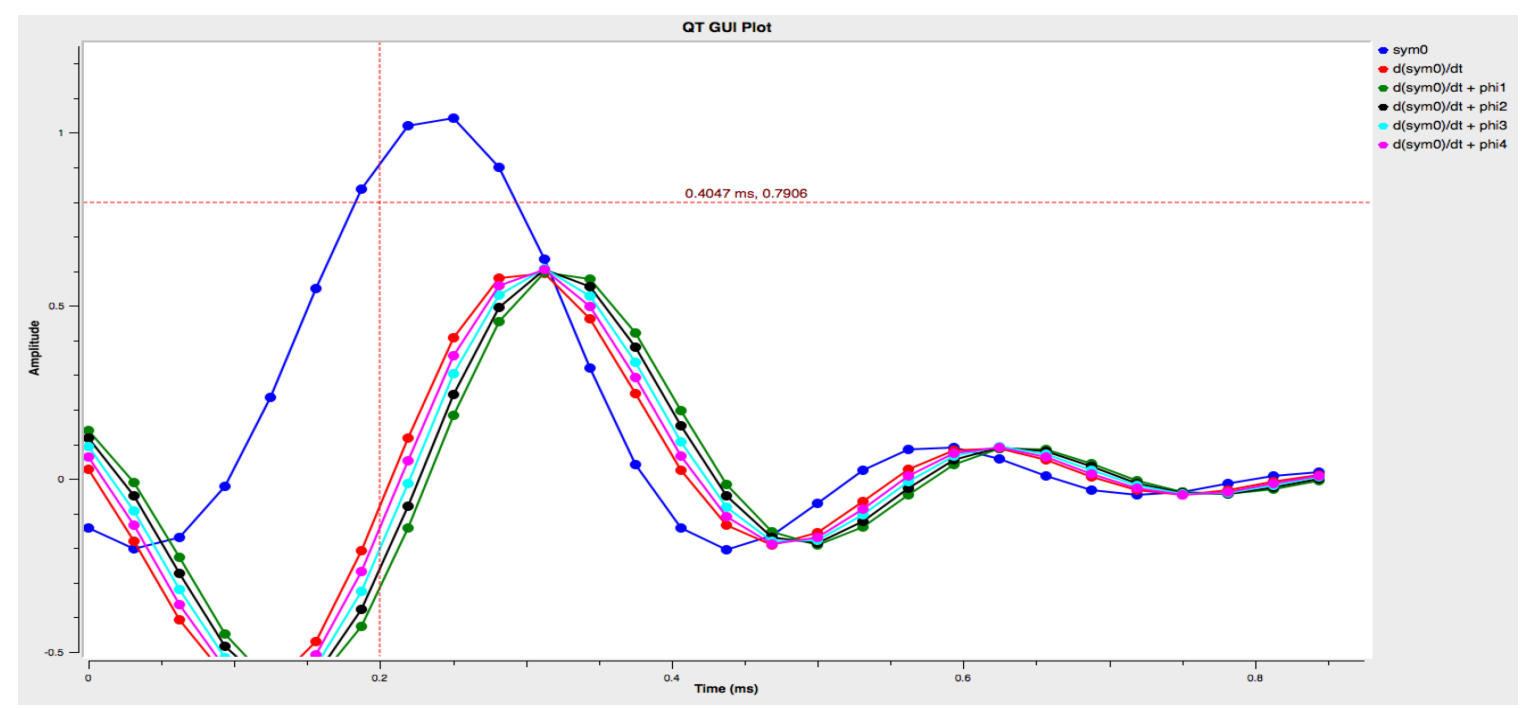

Figure 15: Finding the optimal sampling point using a bank of differential filters.

Finally after time recovery, the signal passes through a Costas Loop block for phase and fine frequency correction. In the next chapter, we see how does the PSK modem perform by simulating the underwater channel in the Arctic-like conditions. 


\subsubsection{Frequency-Shift Keying Modulation}

FSK is another modulation scheme for transmitting digital signals. It has been used for a long time in underwater communications due to its robustness. FSK transmits digital data using a number of frequencies. Each frequency represents different binary pattern. Binary-FSK uses two frequencies. One frequency represents a zero and another represents a one. A FSK waveform is shown in Figure 16. ${ }^{2}$ The symbol time is one symbol per second. The figure also shows the mapping of bits to signals. The upper part shows the transmitted bit sequence, i.e., 1001101. Each bit is mapped to a FSK symbol. At the begining of each symbol, the signal makes a frequency transition. In this example zeros are represented by a low frequency and ones are represented by a high frequency. In the lower part, the amplitude (in volts) of the signal is shown as a function of time (in seconds). The mathematical model for FSK waveform can be found in Goldsmith [38]. In this thesis we introduced FSK informally.

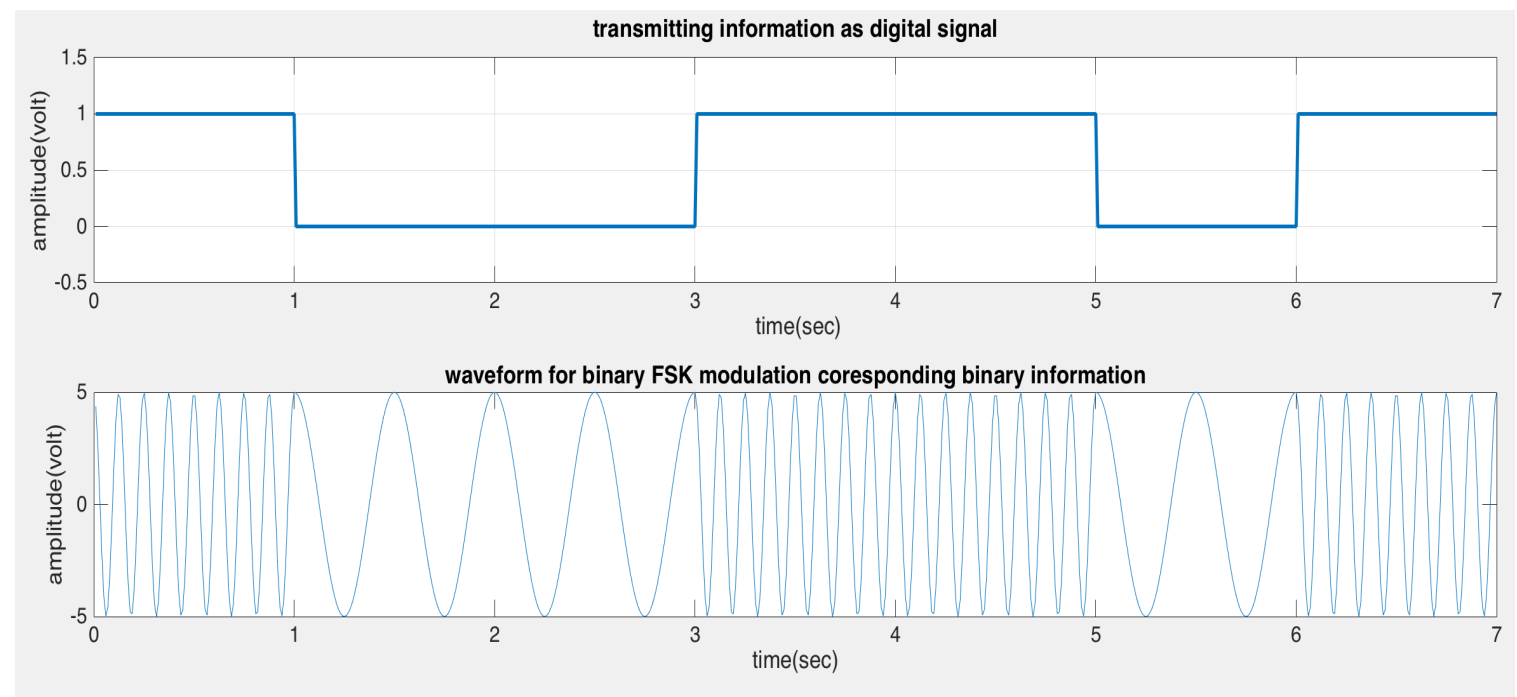

Figure 16: FSK waveform example.

\footnotetext{
${ }^{2}$ Generated using the code adapted from [37]
} 
The FSK transmitter, shown in Figure 17, is fed with data from the file source. The gaussian filter is removed by setting its bandwidth to one. The next step is resampling the signal and shifting the frequency from baseband to passband. Finally, the signal is broadcasted using an audio sink or saved using a .wav file sink.

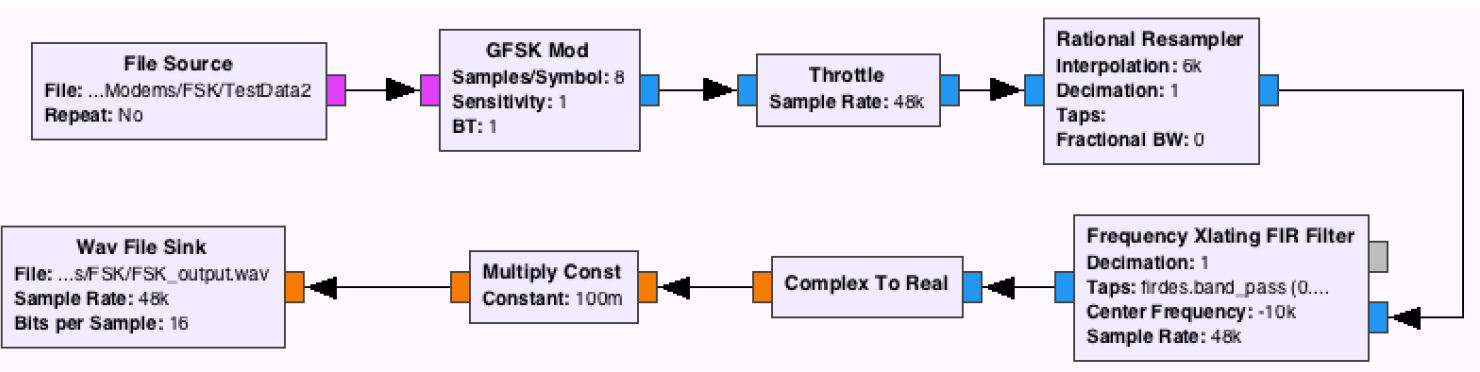

Figure 17: FSK transmitter module.

The FSK receiver, shown in Figure 18, starts by converting the signal from real to complex. The signal is then shifted back to the baseband frequency to be resampled to eight samples per symbol. The signal is then finally demodulated. The data is saved in a text file.

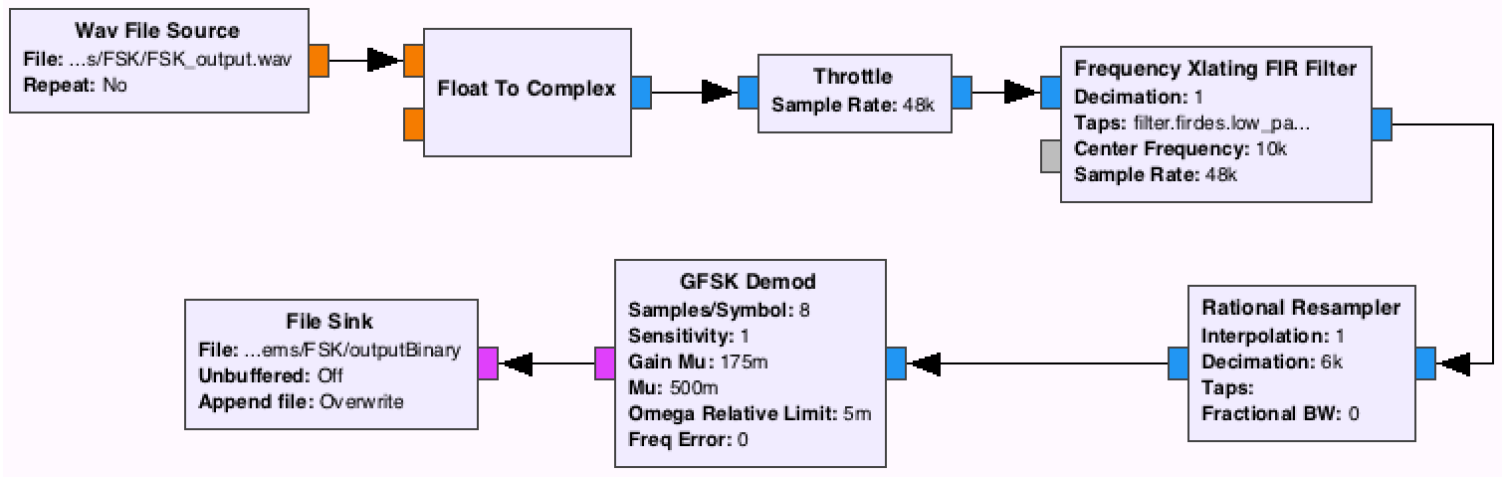

Figure 18: FSK receiver module. 


\subsection{Orthogonal Frequency-Division Multiplexing}

OFDM is a multicarrier modulation scheme. It extends the concept of single carrier modulation by dividing the available bandwidth into sub-bands. Each has its own subcarrier. OFDM utilizes a large number of orthogonal subcarriers that are transmitted in parallel. The approach we took in the single carrier modulation, where we increase the symbol time such that it is longer than the delay spread, effectively reduced the ISI. This limits the data rate. In order to compensate and achieve higher data rates, we elongate the symbol time at each subcarrier. With this approach, several carriers enable transmission at high data rates.

OFDM undergoes InterCarrier Interference (ICI) in the underwater channel. OFDM is sensitive to ICI. Doppler shift is one factor that contributes to frequency offset which causes ICI. Moreover, it gets much worse when Doppler shift is combined with multi path propagation. There are several techniques used in the literature to avoid the ICI due to Doppler spread. Most of the techniques utilize the estimation done on pilot and null carriers, which are spread between data subcarriers. ICI can be mitigated by applying pulse shaping at each subcarrier. In addition to ICI, there exist ISI, which can be mitigated using a guard time interval. The guard time interval is the Cyclic prefix (CP). Finding suitable OFDM symbol time $T$ and guard time $T_{g}$ depends on correct estimation of the delay spread and channel coherence time. The latter can be approximated using the inverse of Doppler spread. 
GNU Radio provides OFDM blocks with several parameters. Two parameters are the number of carriers and their allocation. Other parameters include the Sync words, which are prepended to the OFDM frame and used in the channel estimation. The length of the CP can be defined using the block parameters of OFDM Cyclic Prefixer. The flow graph of the OFDM transmitter can be seen in Figure 19. The figure includes modifications to the original implementation provided by GNU Radio. Those modifications include shifting the frequency to the passband and defining the OFDM block symbol time.
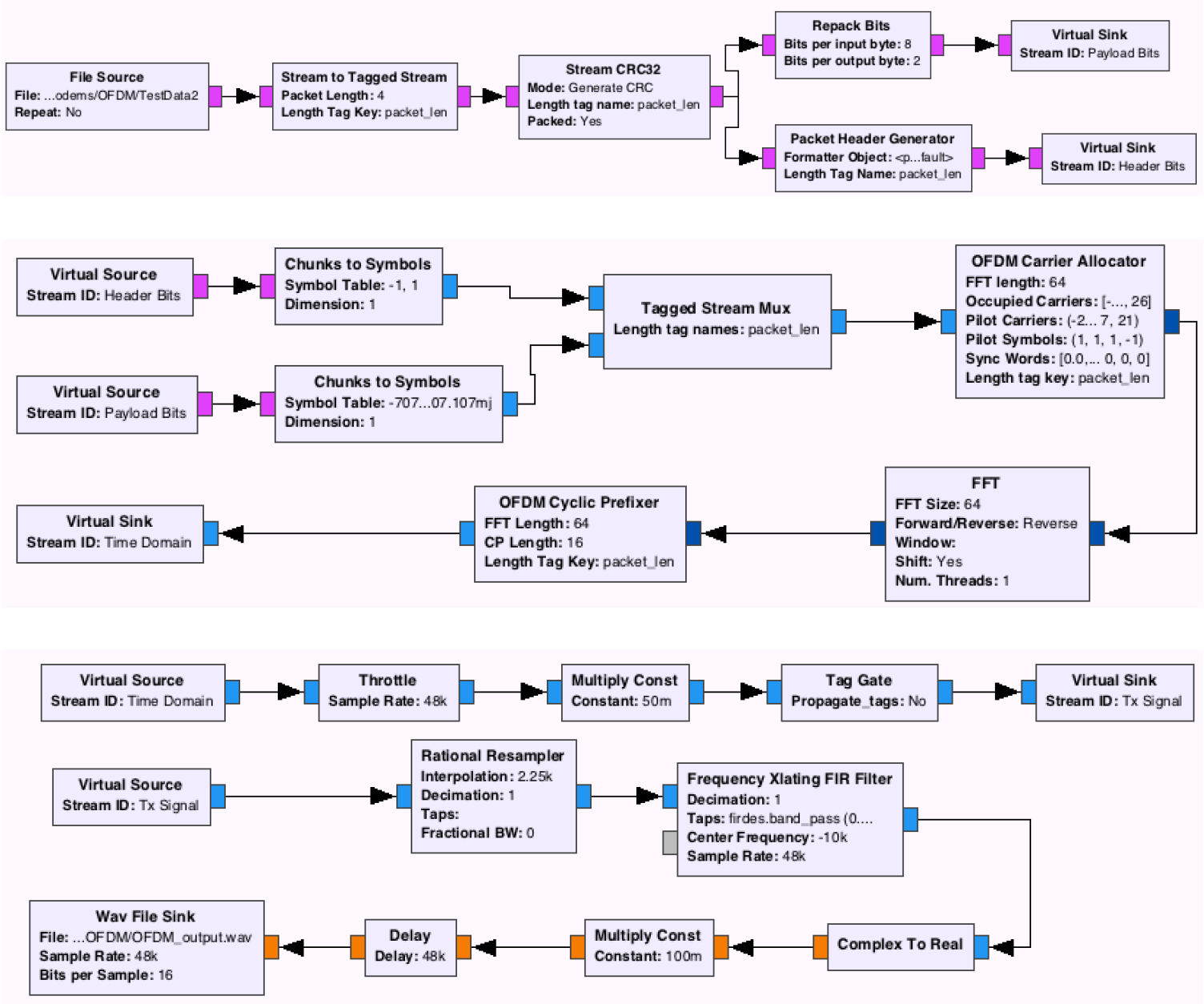

Figure 19: OFDM transmitter module. 
In Figure 19, the data passes through a stream CRC32 block which adds a four-byte CRC to it. The data is then passed to a Packet Header Generator. Each header and payload pair is mapped to BPSK signal for the header and QPSK signal for the payload. The two streams are then combined to form a frame. Next, the OFDM Carrier allocator distributes the modulated symbols along with pilot symbols and prepend them with Sync words. An Inverse Fourier Transform (IFFT) is performed on the output stream of the OFDM Carrier Allocator. Finally, the CP is added. Pulse shaping is performed using the OFDM Cyclic Prefixer block. The time domain stream of the signal is then interpolated and shifted to passband.
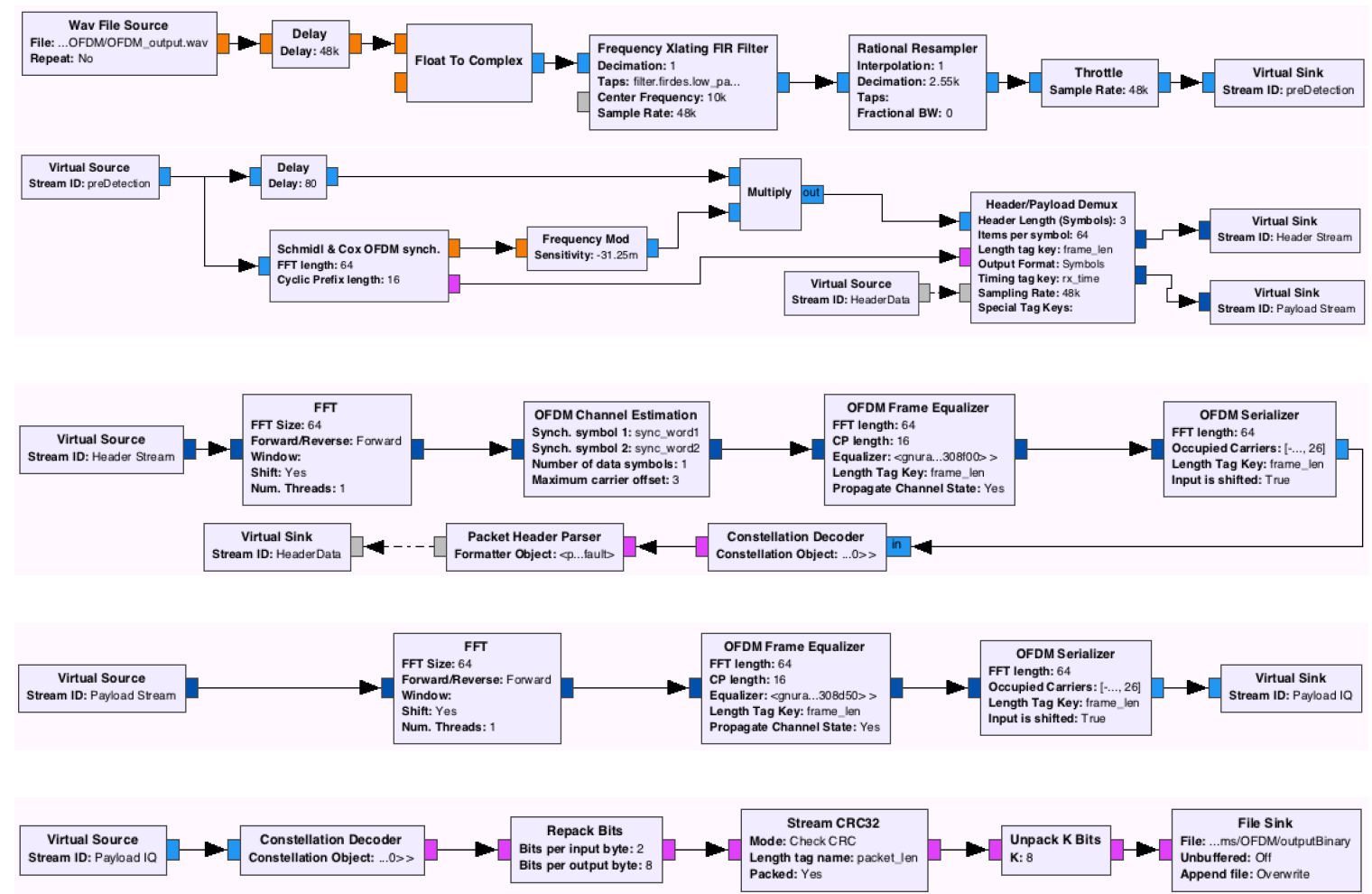

Figure 20: OFDM receiver module. 
Figure 20 shows the GNU Radio flow graph of the OFDM receiver. It starts by receiving signal that is resampled and shifted back to the baseband. The first step is detection and synchronization, which is done using the Schmidl \& Cox OFDM synch block [39]. The Schmidl \& Cox block sends a trigger to the Header/Payload Demux block every time a frame is detected. It sends the header part of the frame to be decoded after channel estimation and equalization is done. It uses the feedback info from the demodulated header signal to output the payload. The payload goes through equalization. It is decoded to output the information sent. 


\subsection{Frequency Hopping-Continuous-Phase Frequency-Shift Keying}

Frequency Hopping CPFSK is another transmission technique used for underwater communications. The frequency for transmission is chosen by the transmitter according to the information bits or a pseudo-random sequence that is known to both the transmitter and the receiver. The receiver is based on energy detection. Ther power of the signal is measured at different frequencies. According to the measurements, the receiver infers what has been sent. Time spreading is not a problem in this technique. However, frequency spreading caused by the underwater channel needs to be mitigated using guard bands between the frequencies.

As shown in Figure 21, time guards are not needed in this technique due to hopping to another frequency upon the transmission of each symbol, therefore clearing all reflections before another transmission occurs on the same frequency. Figure 21 shows an example. The $\mathrm{x}$-axis is time. The $\mathrm{y}$-axis is the frequency domain. There are eight frequencies centered at $F_{c}$. There is one hop per symbol time $\left(T_{s}\right)$. The frequency used at each hop is represented in dark tile. The mathematical model for the waveform can be found in Goldsmith [38]. In this thesis we introduced FH-CPFSK informally. 


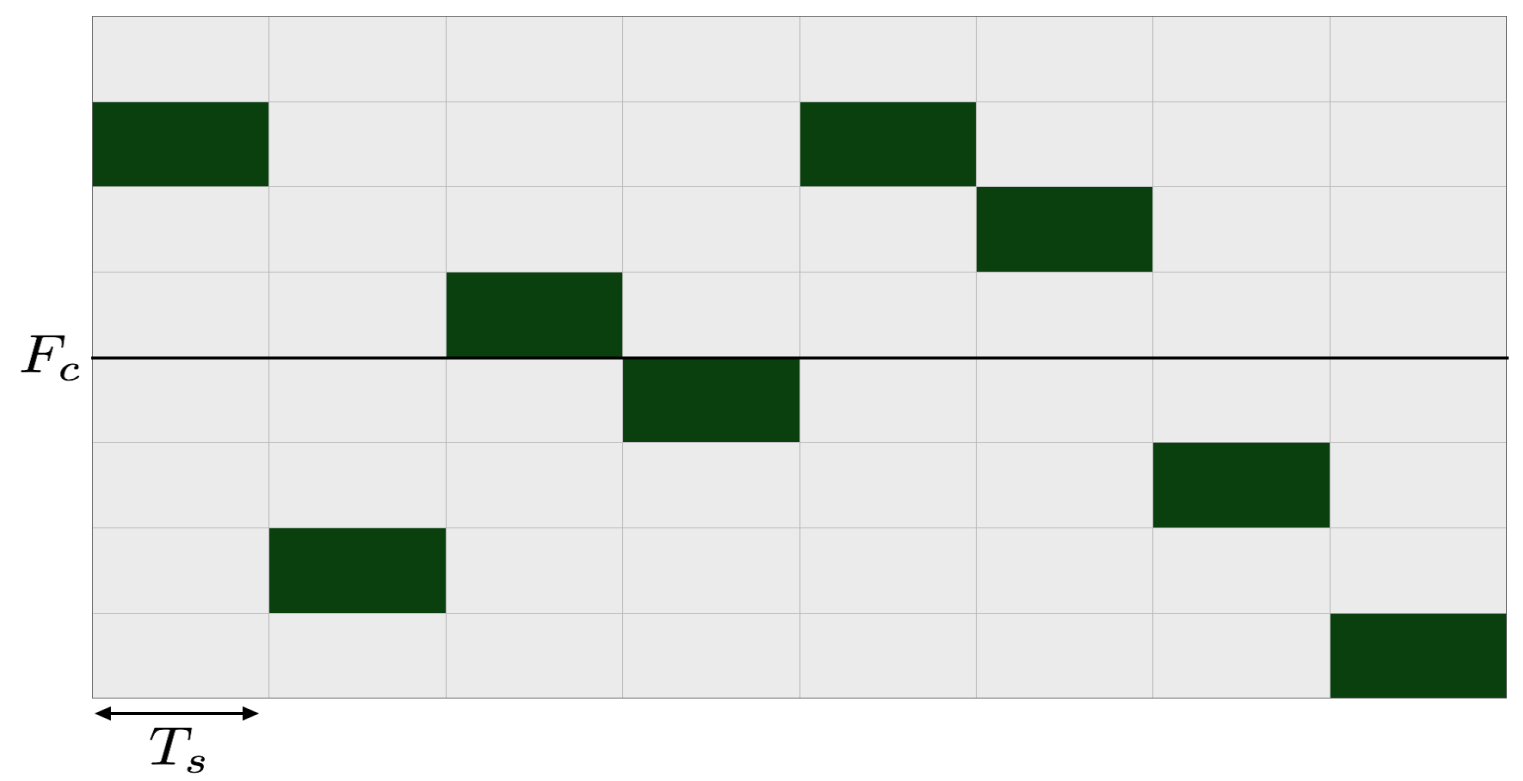

Figure 21: Frequency Hopping CPFSK. 
The GNU Radio flow diagram in Figure 22 represents the Frequency Hopping CPFSK transmitter.It starts by receiving binary data from the File Source block. The data is then modulated using CPFSK modulator block. The frequency synthesizer then shifts the frequency of the signal according to pseudo-random sequence. The signal is then interpolated using the Rational Resampler block. Finally, it is translated using a Frequency Xlating Filter block to the passband. The signal is then saved to a .wav file.

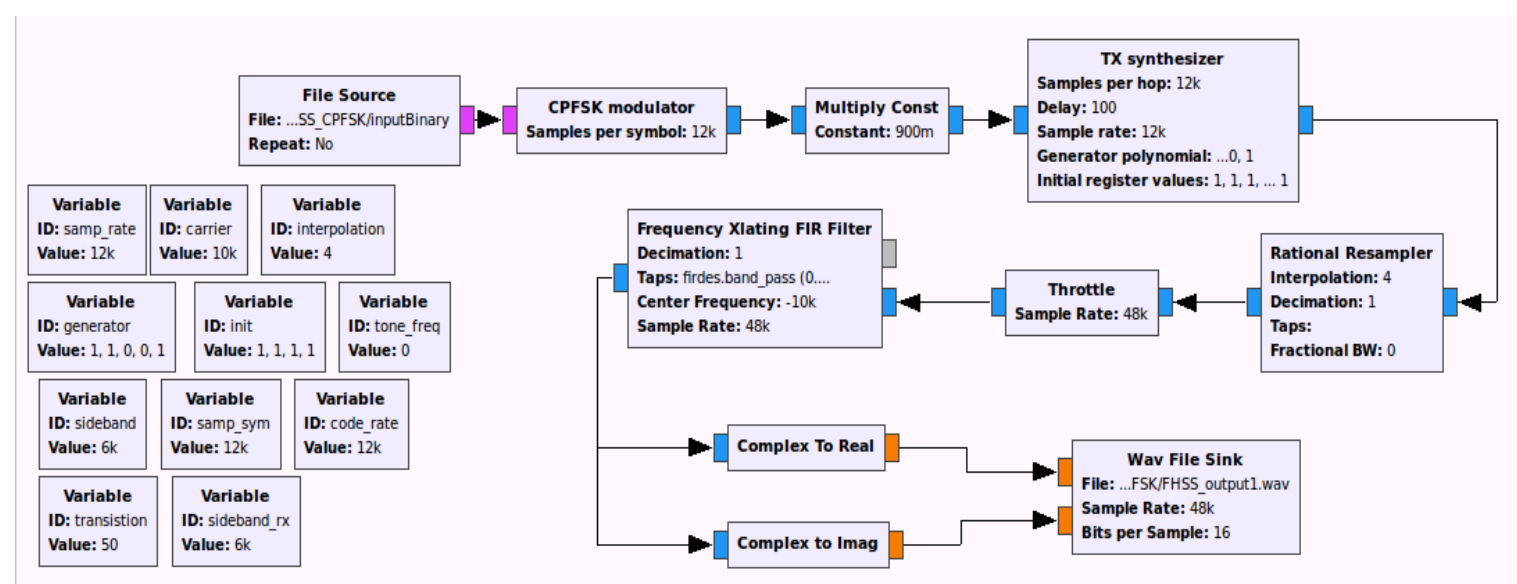

Figure 22: Frequency Hopping CPFSK transmitter module. 
In Figure 23, the Frequency Hopping CPFSK receiver starts by converting the signal from real to complex. The frequency is then shifted back to baseband and down sampled using a Rational Resampler block. The frequency synthesizer is then used to shift back the signal to its original frequency using the same pseudo-random sequence as the transmitter. Finally, the signal is demodulated using an CPFSK demodulator. The implementation of the Frequency Synthesizer block and CPFSK (de)modulator used in the simulator are the work of David [40] and the GNU Radio implementation is downloaded from [41].

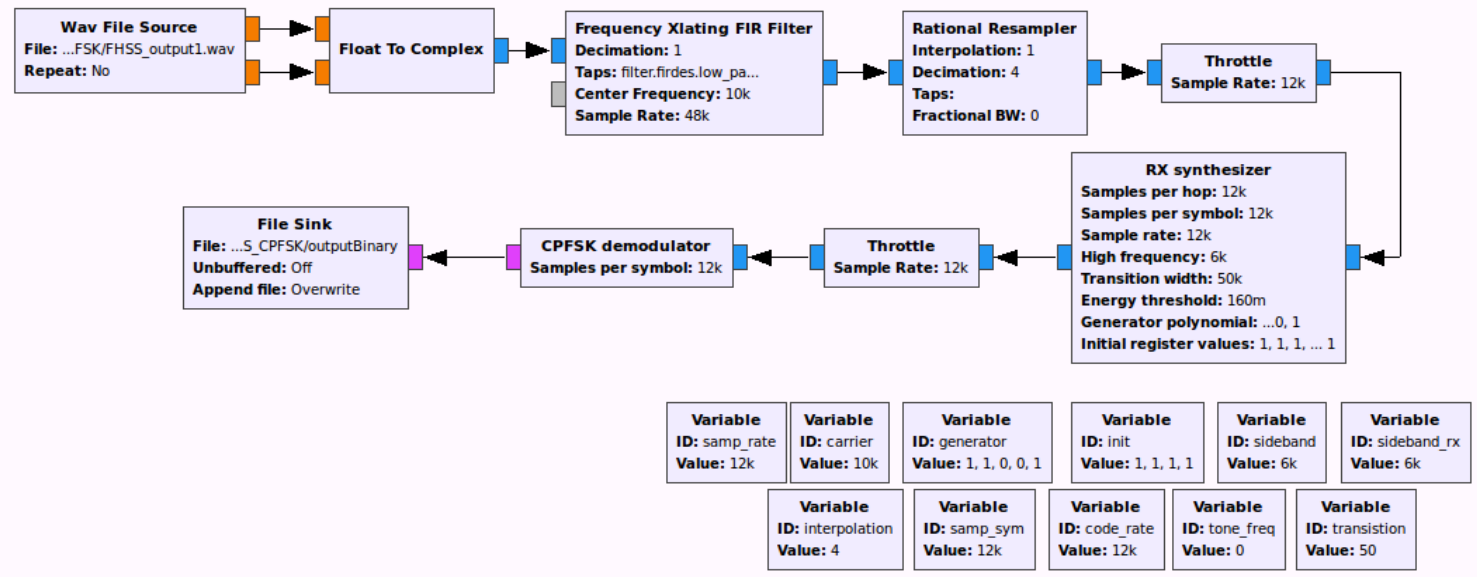

Figure 23: Frequency Hopping CPFSK receiver module.

In the upcoming chapter, simulations are done using the modem designs presented in this chapter. Simulations use the generated .wav files from those transmitters. Afterwards, the signals produced from the channel simulations are used as inputs to our receivers. 


\section{Chapter 5}

\section{Simulation and Results}

\section{$5.1 \quad$ Introduction}

We first describe the simulation methodology. Next, we discuss the simulation environments. Finally, we present the results.

\subsection{Simulation Methodology}

We produced several modems to be evaluated in underwater Arctic-like conditions. Each modem uses a specific modulation scheme and a particular transmission technique. An evaluation is needed to determine the best strategy to use for communications in Arctic-like conditions. Therefore, an evaluation of these techniques is presented in this chapter. The results can be utilized for selecting the most suitable modulation scheme for Arctic-like conditions..

The simulations done consider different environments. For each environment, we perform simulations using all of the modulation techniques presented in this thesis. We compare their performance. The performance metric is the Bit Error Rate (BER) vs Energy per Bit to Noise power spectral density ratio $E_{b} / N_{0} . E_{b} / N_{0}$ is also known as SNR per bit, which is a normalized SNR measure. It is a good metric for comparing the performance of different modulation schemes. For OFDM vs. other modulation techniques, we compare the Packet Error Rate (PER) vs SNR.

The simulation uses three main modules. These modules are the modulator, channel and demodulator. Figure 24 shows the architecture of the simulation. We first generate the signals using the modems designed in GNU Radio and save them into .wav 
files. Next, we convolve the signals and impulse responses of the generated environments using the BELLHOP software and MATLAB ${ }^{\mathrm{TM}}$ to produce new .wav files. We then add white Gaussian noise using MATLAB ${ }^{\mathrm{TM}}$. The resulting .wav files are then demodulated using the modems designed in GNU Radio to produce the output files. Finally, the input and output files are compared and the BERs or PERs are calculated.

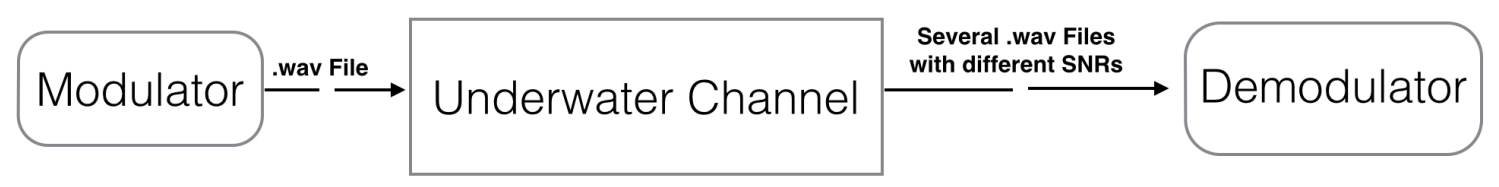

Figure 24: Architecture of the simulator.

\subsection{Impulse Response}

The impulse response is generated using the modified version of the BELLHOP software. BELLHOP was modified to distinguish between rays reflecting from the underside of ice versus those originating from the air-sea interface [16]. In addition, the altimetry files that represent the ice cover has been supplied to us by Maritime Way Scientific. Using BELLHOP, one can generate an underwater environment to be simulated and produce an arrival file. The arrival file defines the impulse response. The configuration of the environment file and use of BELLHOP to produce the arrival files are explained in details in [15]. Figure 25 shows an example of an environment file. We are capable of defining the frequency of the signal, sound speed profile, altimetry and bathymetry files, range of the source and destination, depth of the source and receiver, seabed and angles of the source beam. The ice cover is specified in an altimetry file. 


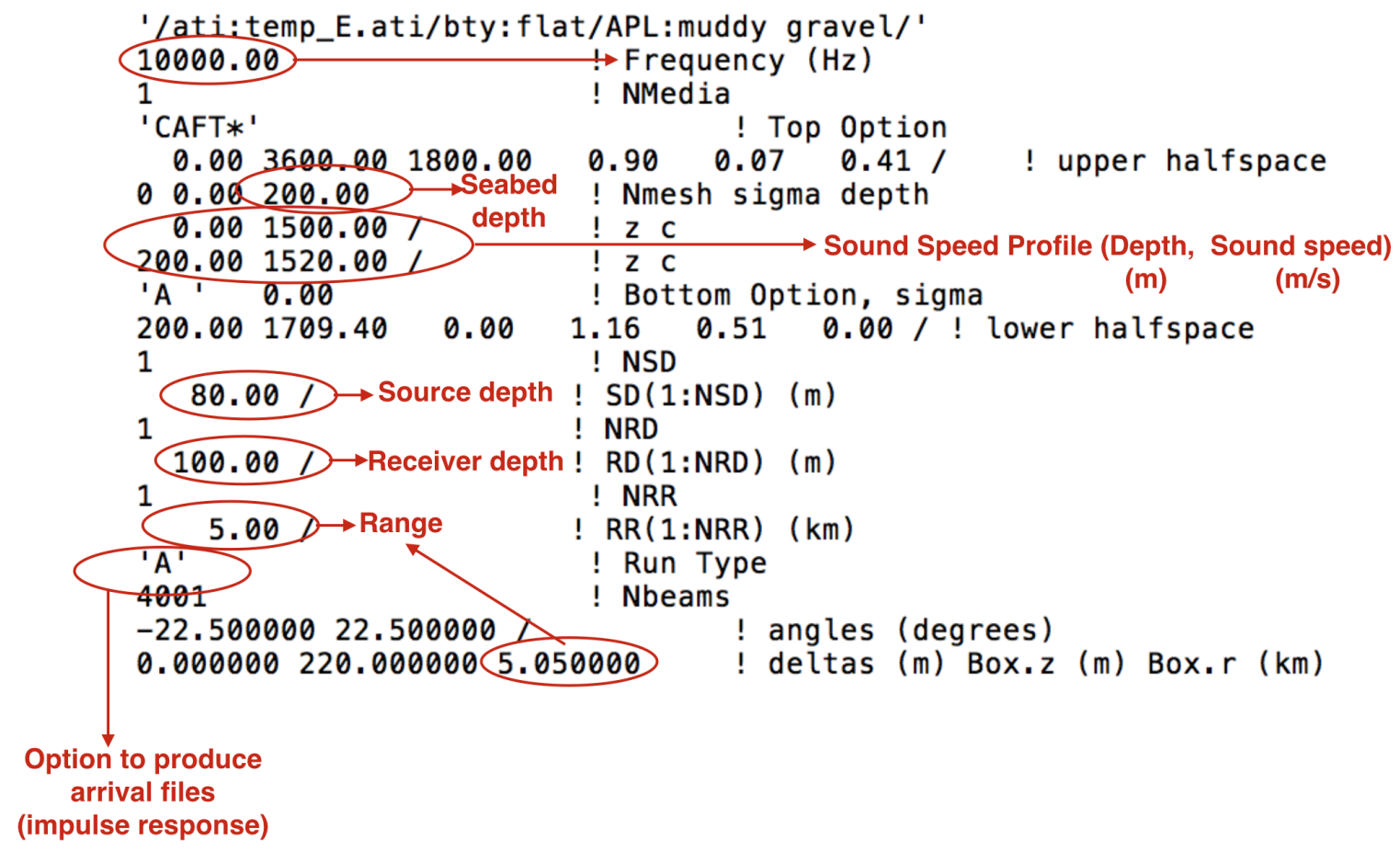

Figure 25: An example of an environment file. 


\subsection{Simulation Environments}

Simulation environments were produced using the BELLHOP modified version and MWS' ice covers. We focus on two main categories of environments. The first category consist of environments with different ice cover percentages. We look at how each of the modulation techniques performs with different ice-cover percentages. The ice modelling is based on a paper by Goff [42]. The partial ice cover produced using the technique explained in [16], in which a full range ice cover is first produced. Next, random length sections of the ice cover are removed at random starting points until the desired ice cover percentage is reached. We look at environments with $0 \%, 20 \%$, $40 \%$ and $80 \%$ ice cover, all with an upward refracting sound speed profile as presented in Figure 26. It shows the speed of the sound as a function of depth. It grows linearly from $1500 \mathrm{~m} / \mathrm{s}$ near sea surface up to $1520 \mathrm{~m} / \mathrm{s}$ near seabed. The reason for upward refraction is due to the presence of sea ice cover, in which it insulates the Arctic ocean from solar heating [43]. As a result the speed of sound increases with depth causing an upward refracting environment.

In the case of environments with ice cover, for each of the ice percentages we use between 30 and 100 files of different environments with different actual ice covers. Figures 27, 28, 29 and 30 show respectively samples of $0 \%, 20 \%, 40 \%$ and $80 \%$ ice covers. To understand each of the figures, the x-axis shows the range between the transmitter and the receiver. The y-axis shows the depth. The transmitter and receiver are 80 and 100 meters deep, respectively. The seabed is 200 meters deep. The lines and curves are eigenrays connecting the transmitter and receiver. Red curves are showing eigenrays that have changed direction due to refraction. Blue and black lines and curves show the eigenrays that are reflected by the seabed, sea surface or both. The ice cover is represented in red along the horizontal top line. With $80 \%$ ice cover, there is a significant increase in the number of eigenrays which translates in more arrivals at the receiver.

In the second category, we look at how the modulation techniques perform with respect to distance. We take two different ranges: $5 \mathrm{~km}$ and $10 \mathrm{~km}$. Both environments have $20 \%$ of ice cover. Figure 31 shows a sample for the $10 \mathrm{~km}$ range. With respect to Figure 30, there is an enormous increase in the number of eigenrays. 


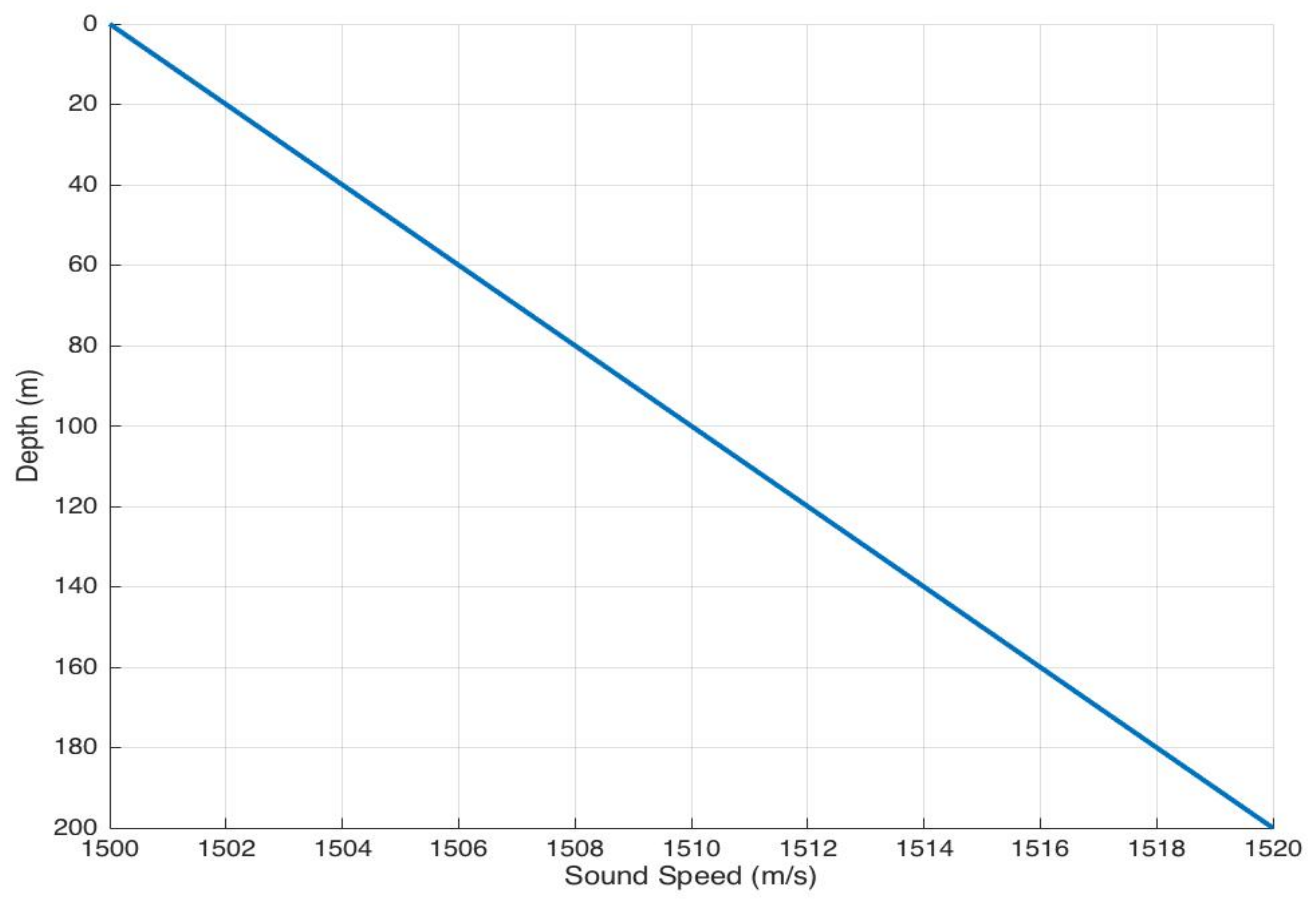

Figure 26: Upward refracting sound speed profile.

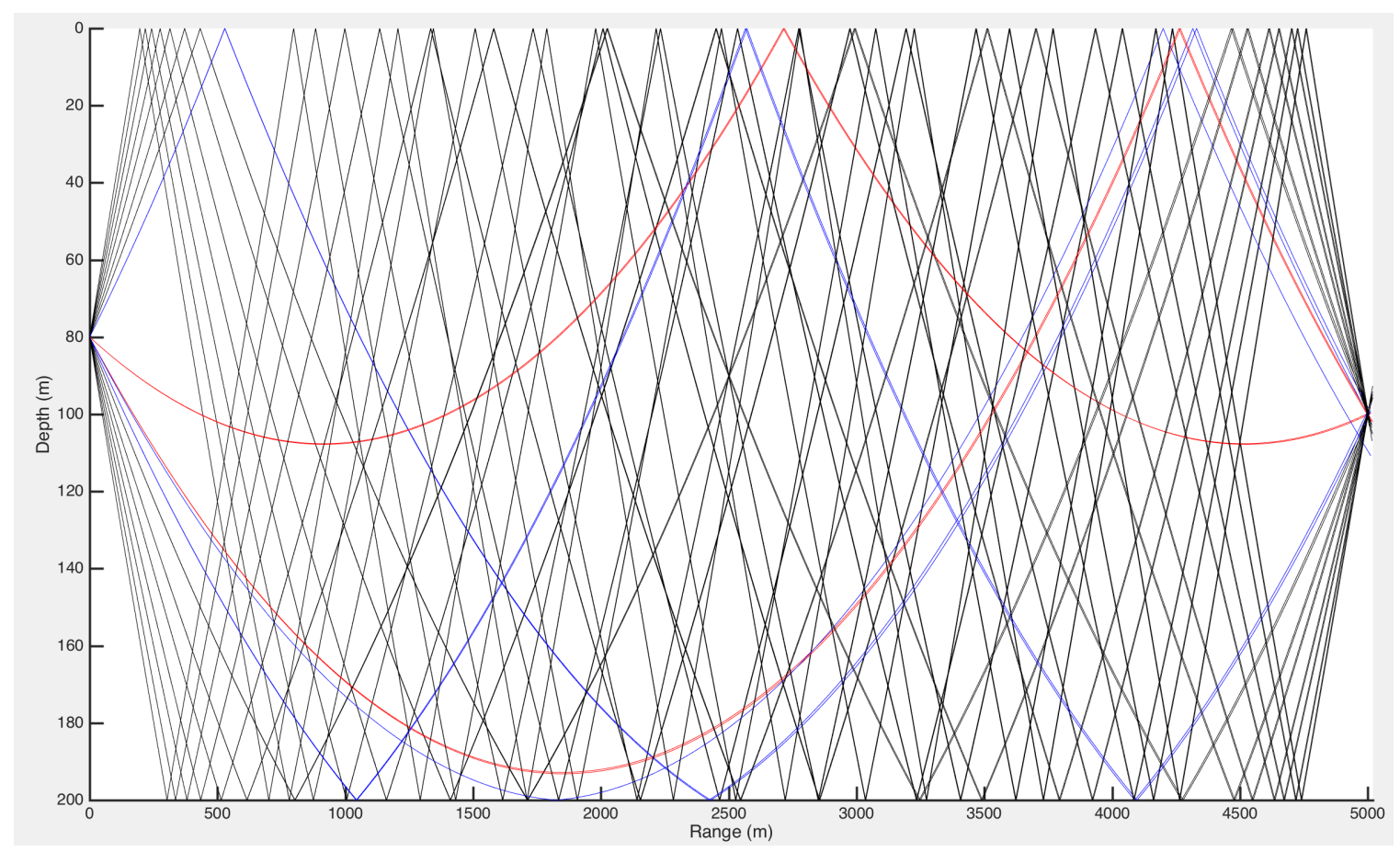

Figure 27: Environment sample with $0 \%$ ice cover. 


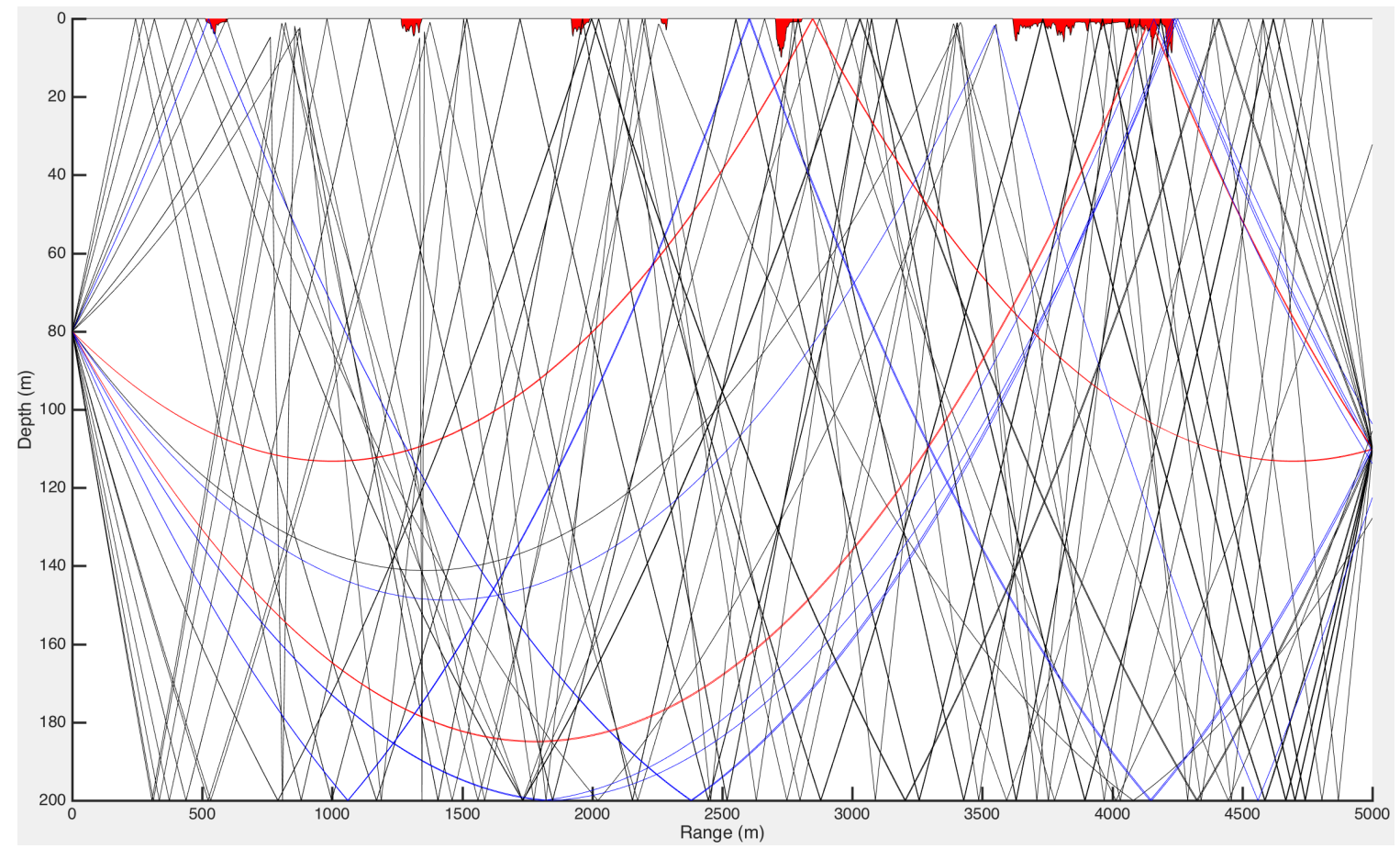

Figure 28: Environment sample with $20 \%$ ice cover.

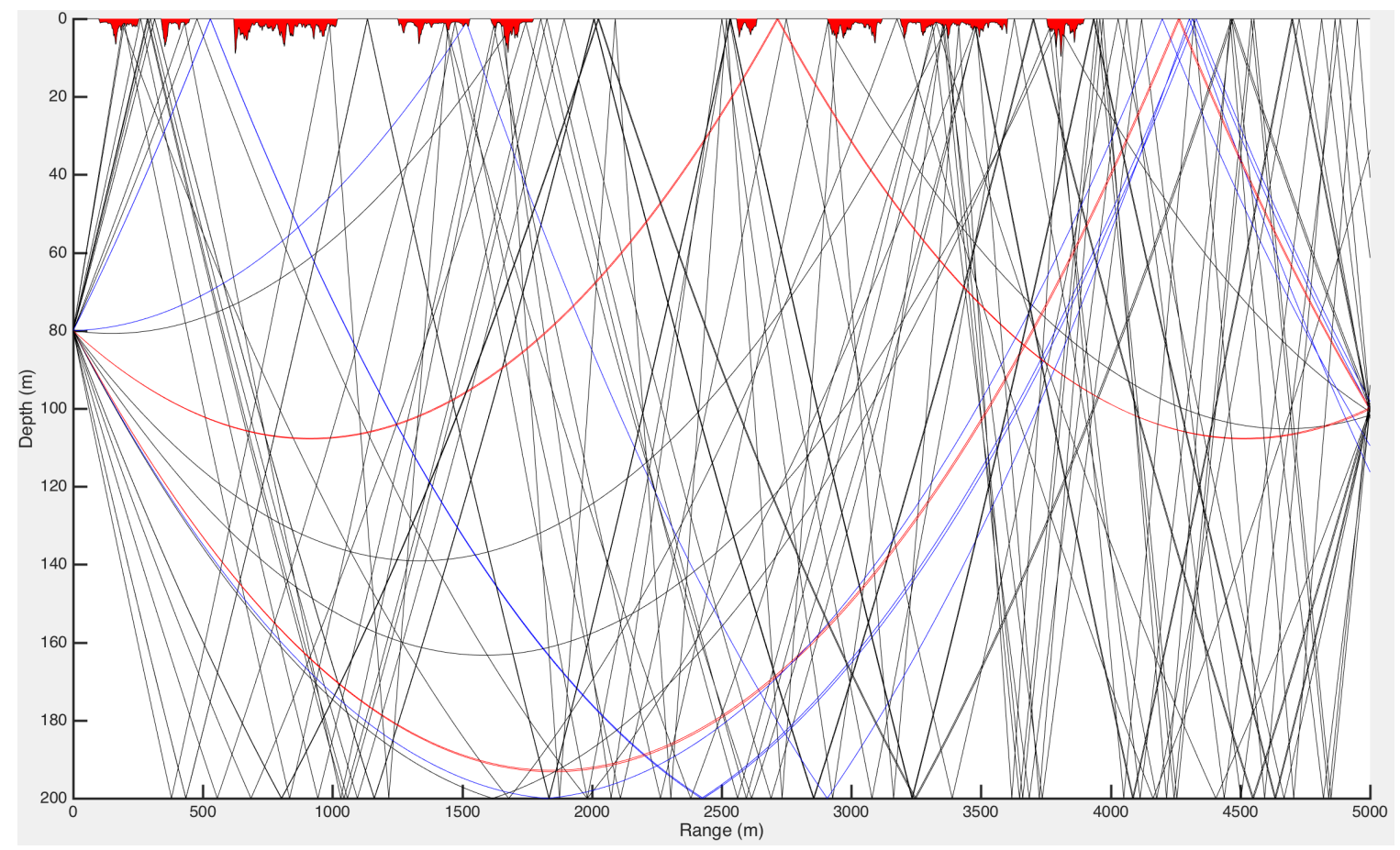

Figure 29: Environment sample with $40 \%$ ice cover. 


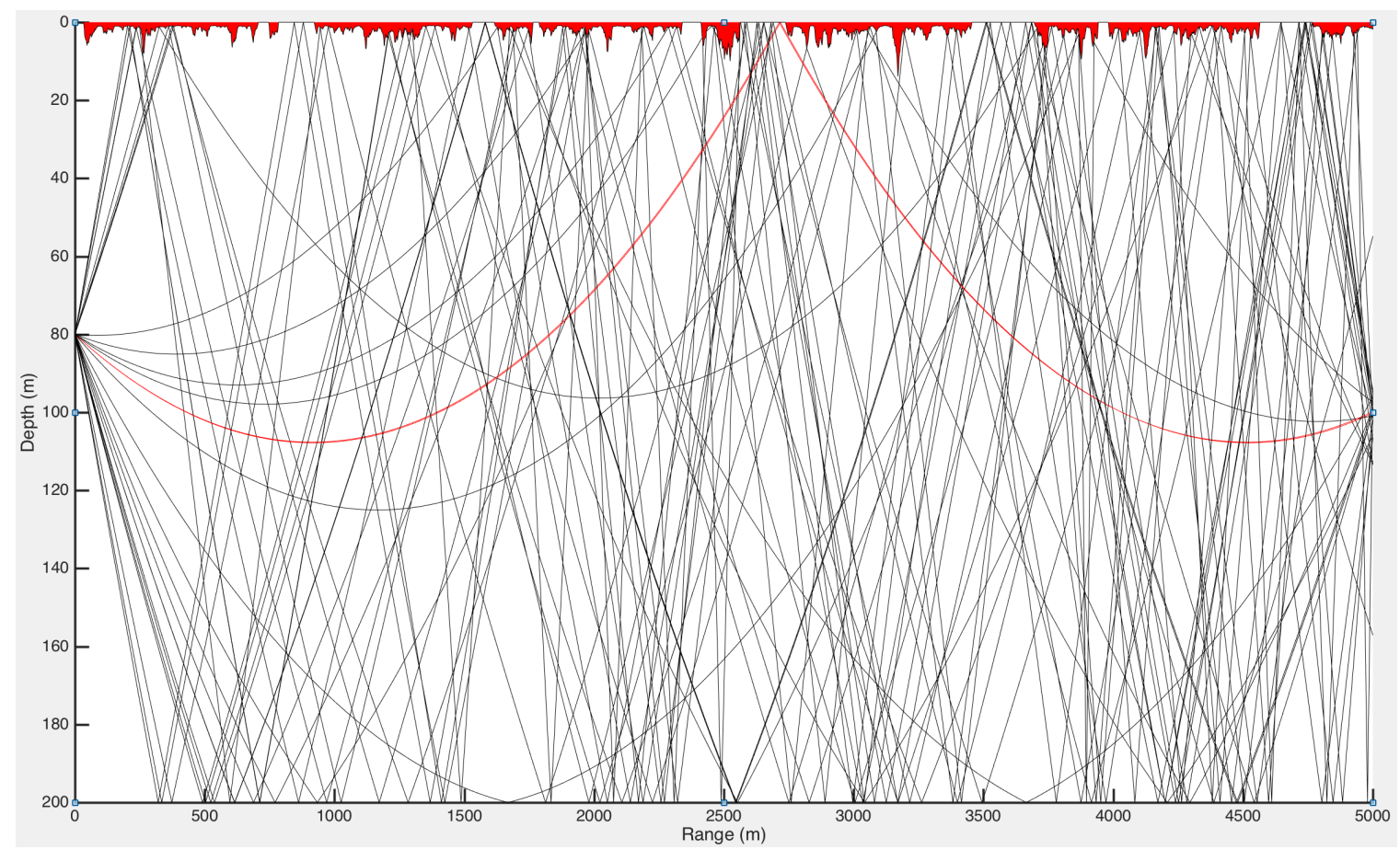

Figure 30: Environment sample with $80 \%$ ice cover.

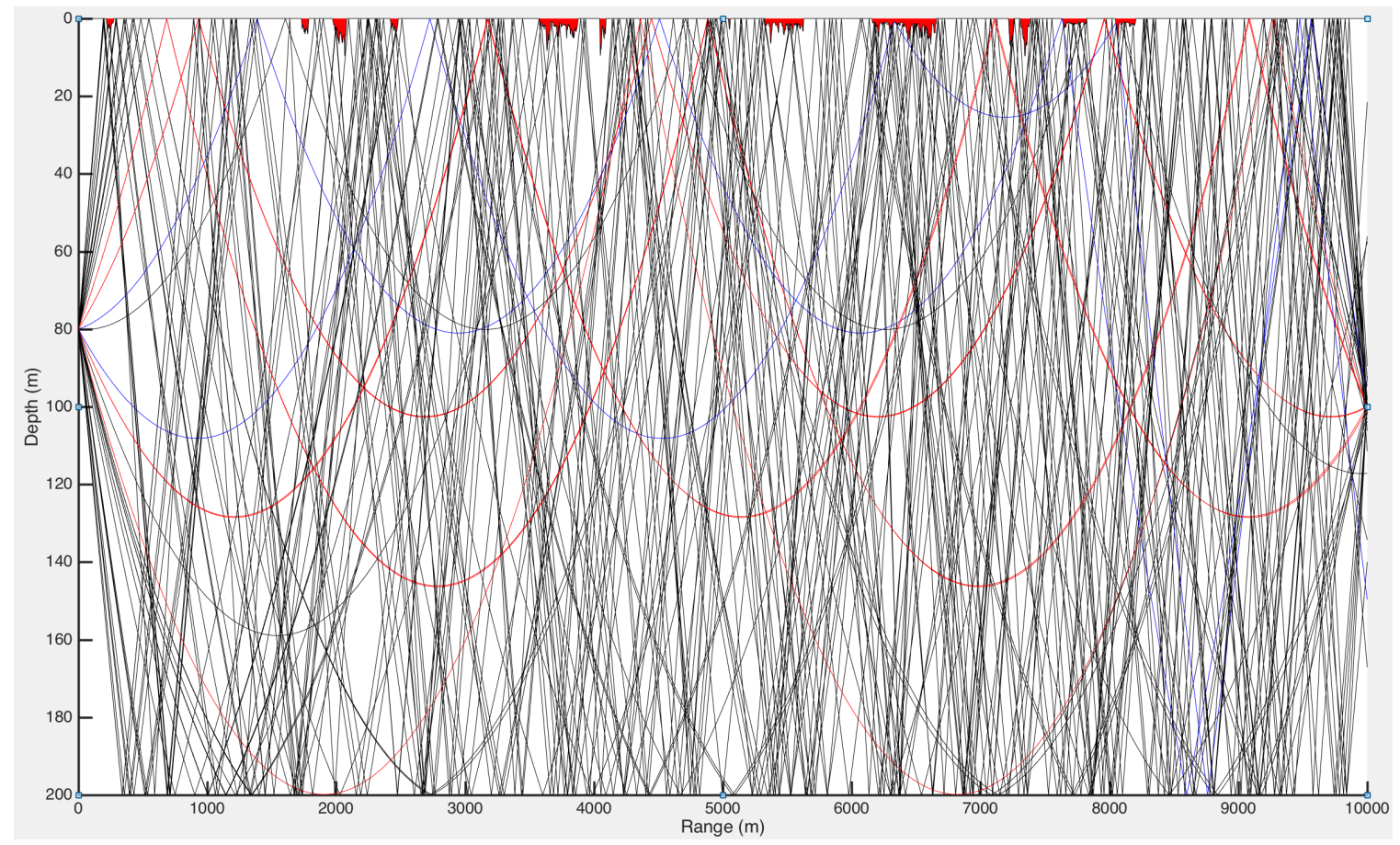

Figure 31: Environment sample with 20\% ice cover and $10 \mathrm{~km}$ range. 


\subsection{Measure of Variability}

In this section, we look at the delay spread generated by each of the environments. We measure the variability of the delay spread of each of the ice percentages using InterQuartile Range (IQR). IQR is a measure of statistical dispersion [44]. The IQR is calculated by first sorting the data set in increasing order. The data set is then cut into four equal parts. The quartiles are the numbers at the cuts. There are three quartiles. The IQR is calculated by subtracting the first quartile from the third quartile. Figures 32, 33, 34 and 35 show the IQR of 100 files for the four different ice cover percentages. They all show that the IQR starts to converge as the number of files approach 30. Which is actually the minimum number of files that we are using for each ice cover.

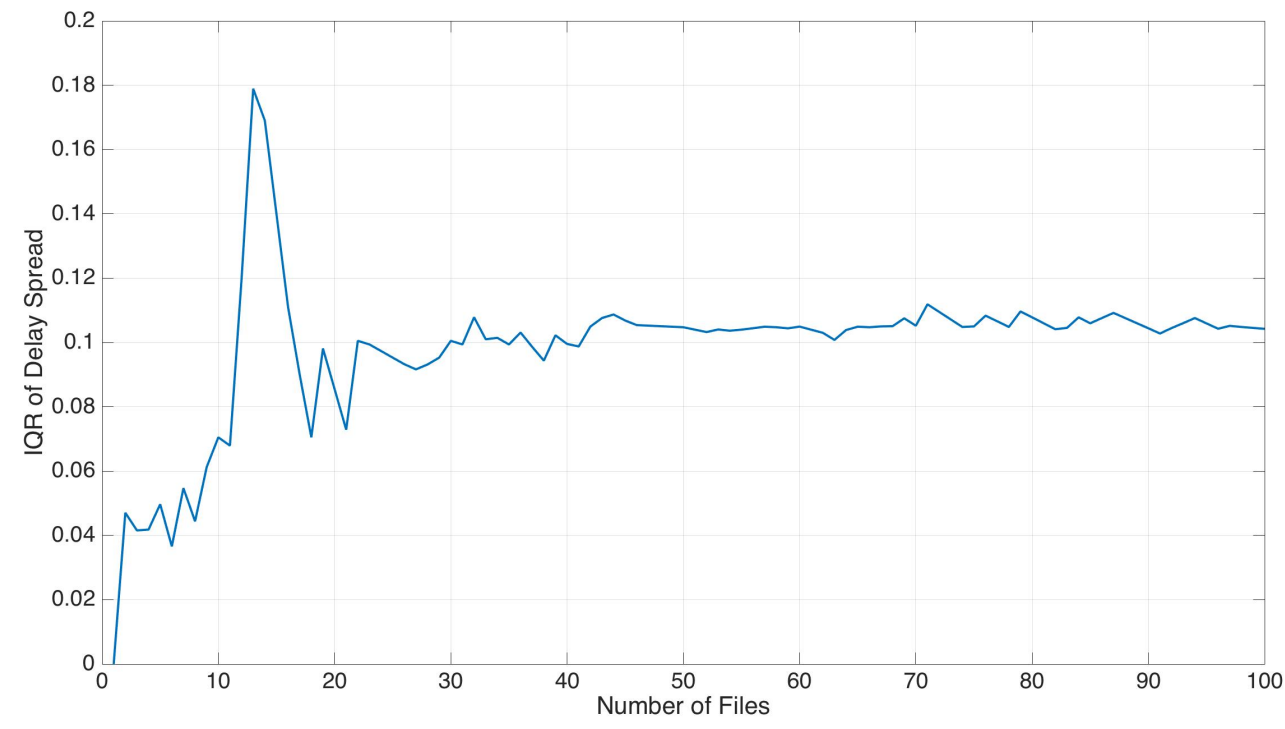

Figure 32: IQR for 100 files with 20 percent ice cover and $5 \mathrm{~km}$ in range. 


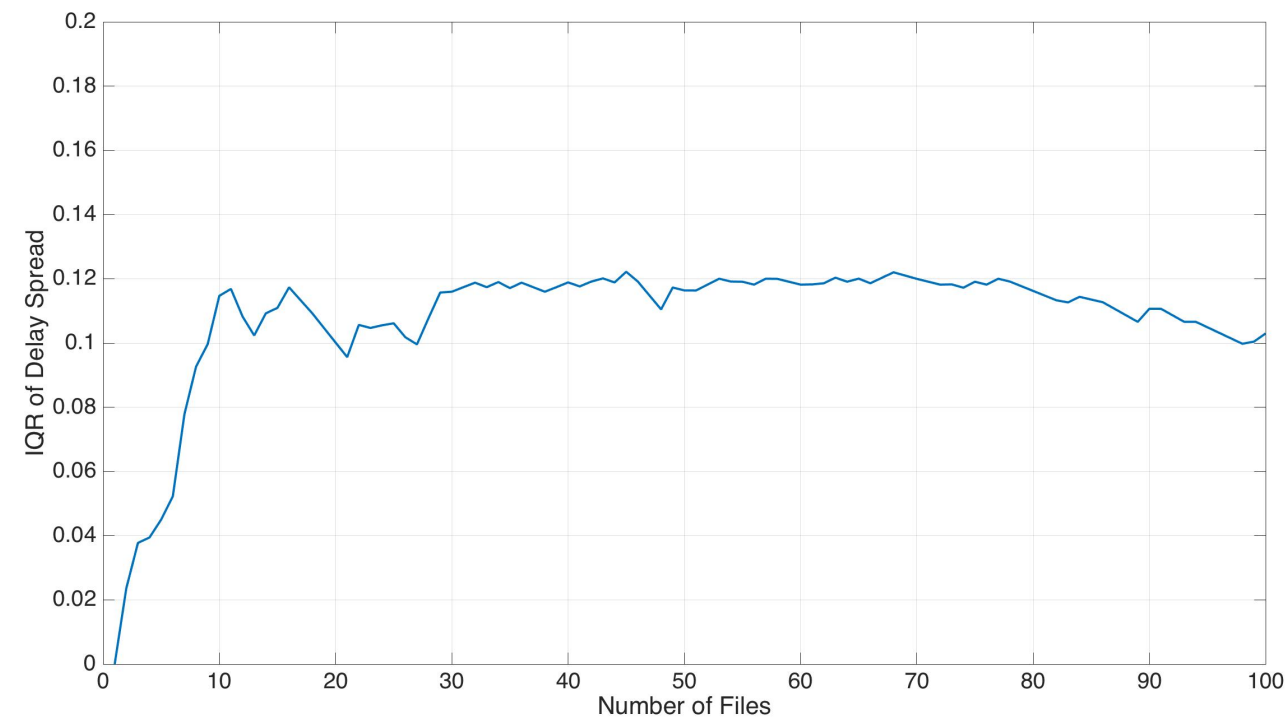

Figure 33: IQR for 100 files with 40 percent ice cover and $5 \mathrm{~km}$ in range.

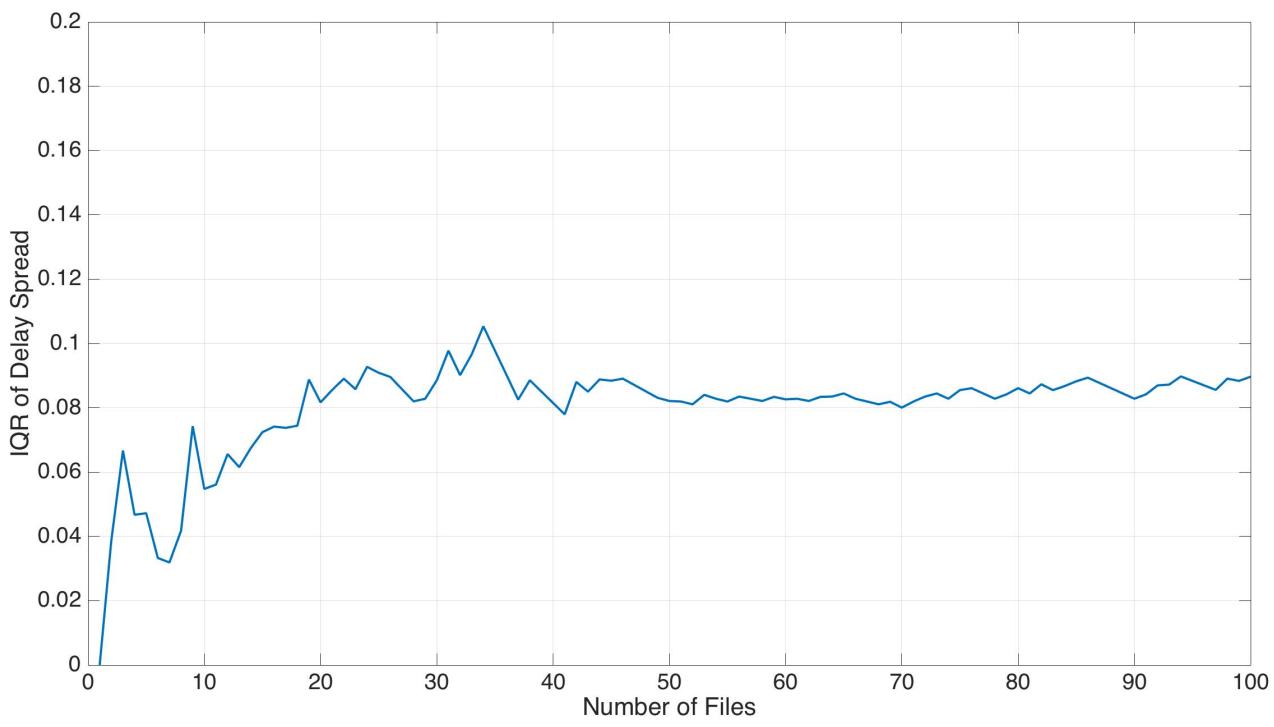

Figure 34: IQR for 100 files with 80 percent ice cover and $5 \mathrm{~km}$ in range. 


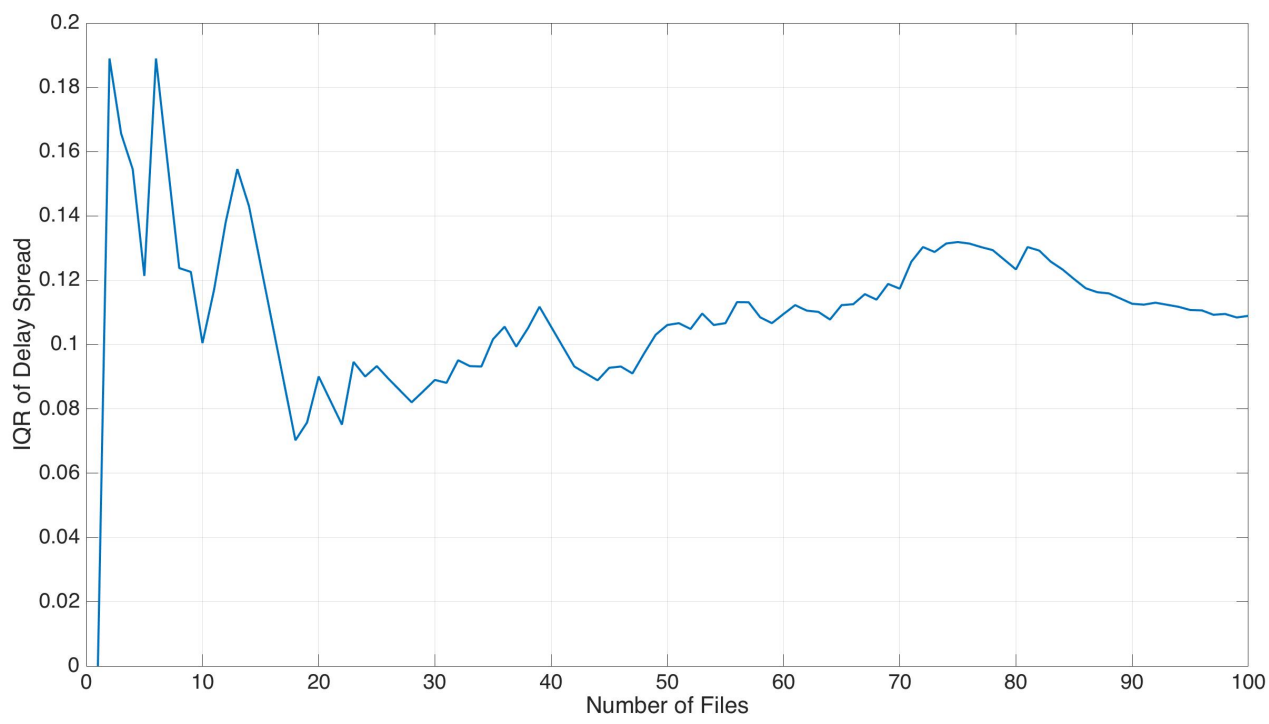

Figure 35: IQR for 100 files with 20 percent ice cover and $10 \mathrm{~km}$ in range. 


\subsection{Delay Spreads}

The simulations consist of transmitting 800 bits through these different environments we have mentioned. The center frequency used is $10 \mathrm{KHz}$. For each of the modulation techniques, we use three baud rates in the $5 \mathrm{~km}$ and $20 \%$ ice cover environments. The baud rates are three, one and one third of a symbol per second. While we only a baud rate of one third of a symbol per sec in the environments with $40 \%$ and $80 \%$ ice cover. We produce 17 .wav file from each environment. Each of the .wav files has different SNRs ranging from -50 up to $30 \mathrm{~dB}$. We observed an increase in the average delay spread as ice cover percentage increased. However with longer distance the delay spread increases much more. The average delay spreads are 0.4446, 0.4552,

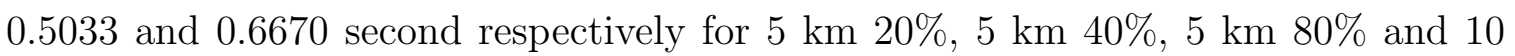
$\mathrm{km} 20 \%$ ice cover.

The delay spreads of each file in the aforementioned environments are shown in the Figures 36, 37, 38 and 39. Some outliers can be seen in the figures.

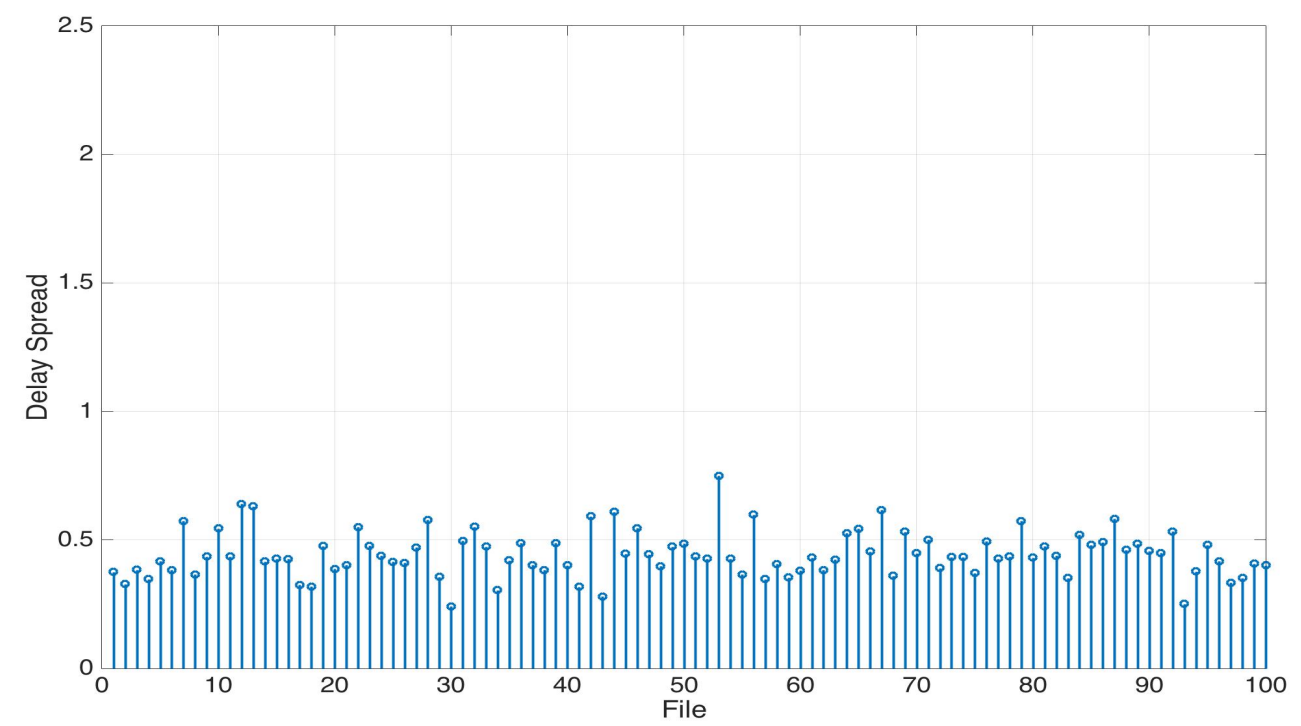

Figure 36: The delay spread of each of the 100 files with $20 \%$ ice cover and $5 \mathrm{~km}$ in range. The average of the delay spreads equals to 0.4446 , the standard deviation equals to 0.0884 and the median equals to 0.4336 . 


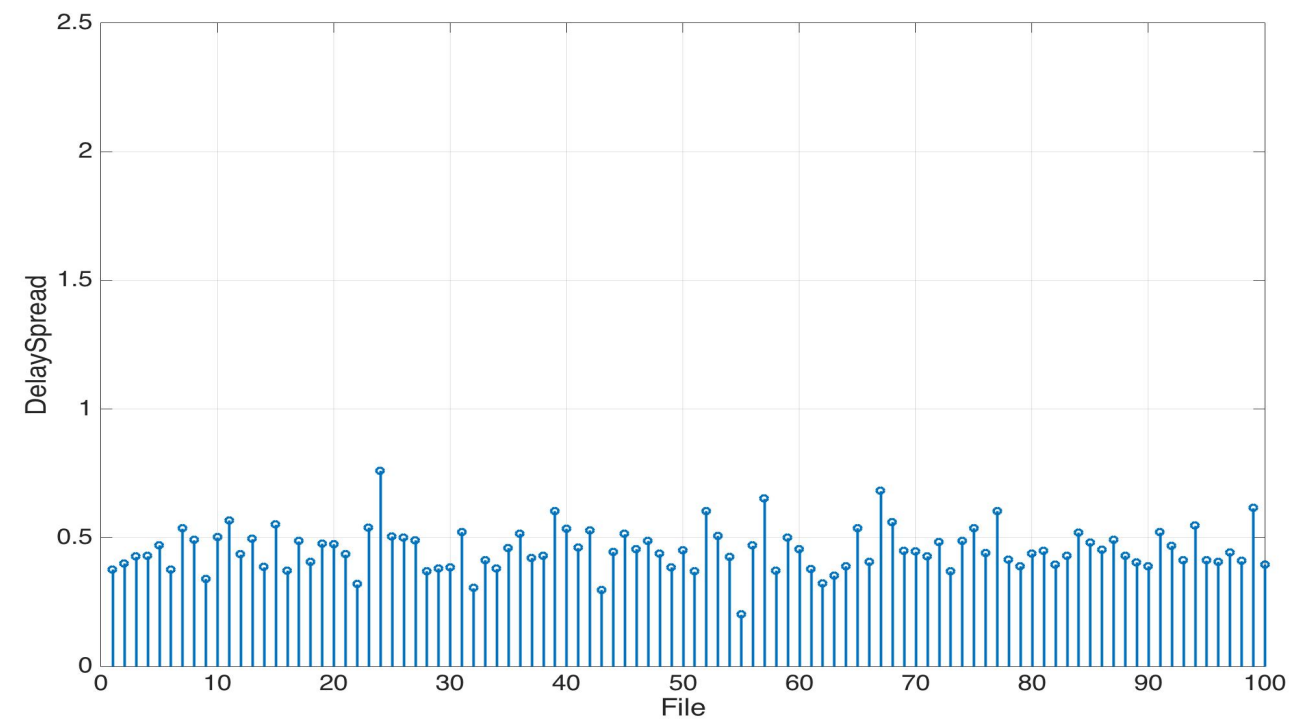

Figure 37: The delay spread of each of the 100 files with $40 \%$ ice cover and $5 \mathrm{~km}$ in range. The average of the delay spreads equals to 0.4552 , the standard deviation equals to 0.0841 and the median equals to 0.4458 .

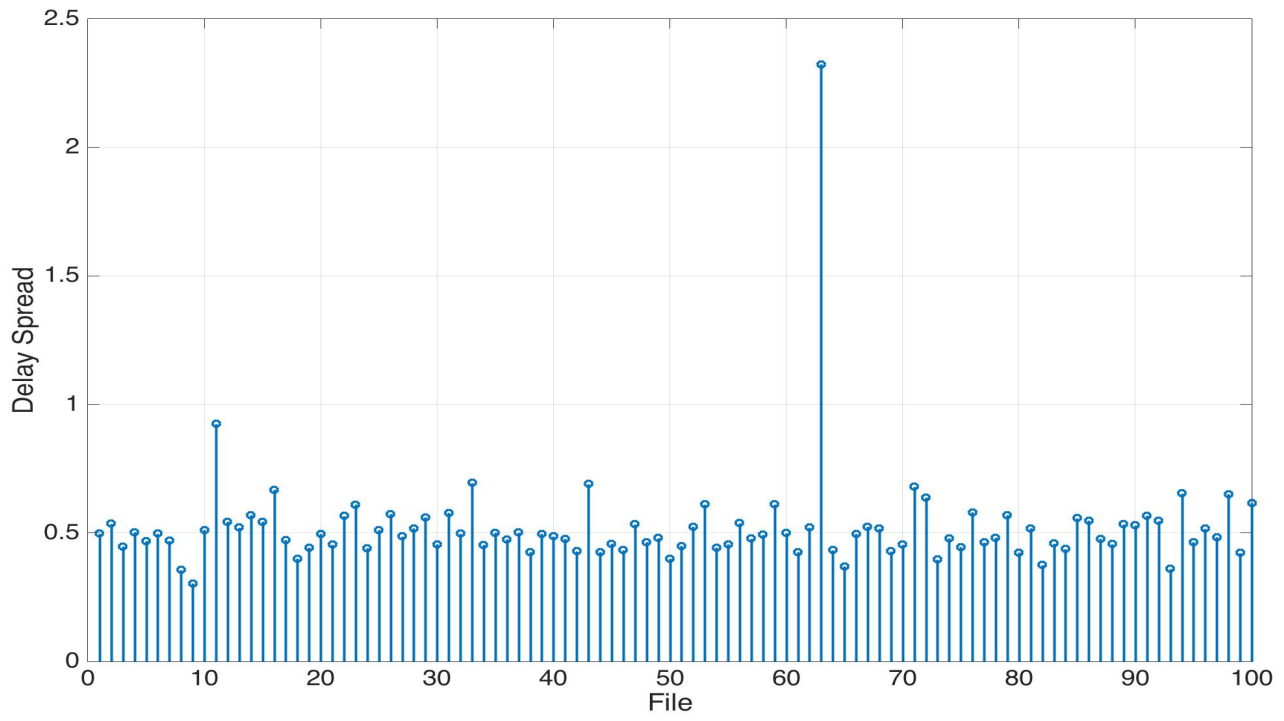

Figure 38: The delay spread of each of the 100 files with $80 \%$ ice cover and $5 \mathrm{~km}$ in range. The average of the delay spreads after removing the outlier equals to 0.5034 , the standard deviation equals to 0.0861 and the median equals to 0.4949 . 


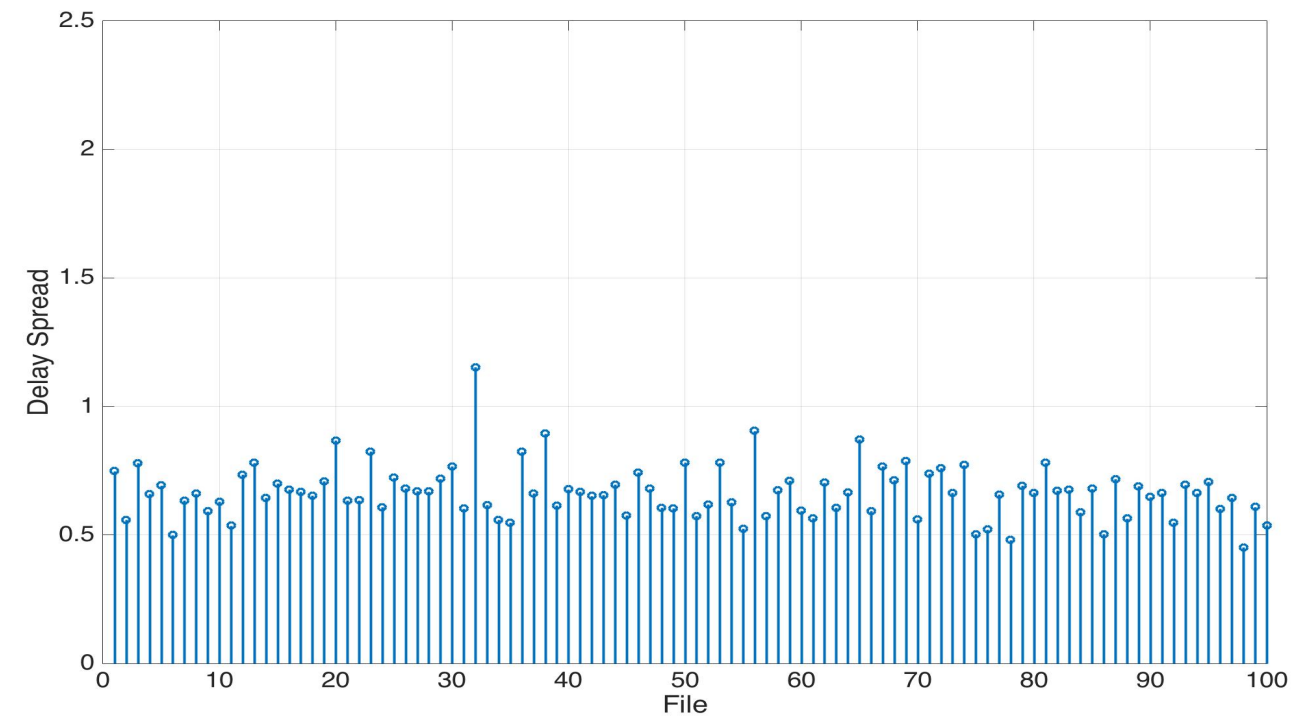

Figure 39: The delay spread of each of the 100 files with $20 \%$ ice cover and $10 \mathrm{~km}$ in range. The average of the delay spreads equals to 0.6670 , the standard deviation equals to 0.1032 and the median equals to 0.6639 . 


\subsection{Results}

We first show the results of each modulation scheme and transmission technique alone, with different baud rates. They are chosen such that one baud rate has a symbol time $\left(T_{s}\right)$ less than the delay spread, another baud rate has a symbol time that is slightly greater than the delay spread and the third baud rate has a symbol time that is much greater than the delay spread. The confidence intervals plots can be found in Appendix A.

\subsubsection{Binary Phase-Shift Keying Performance}

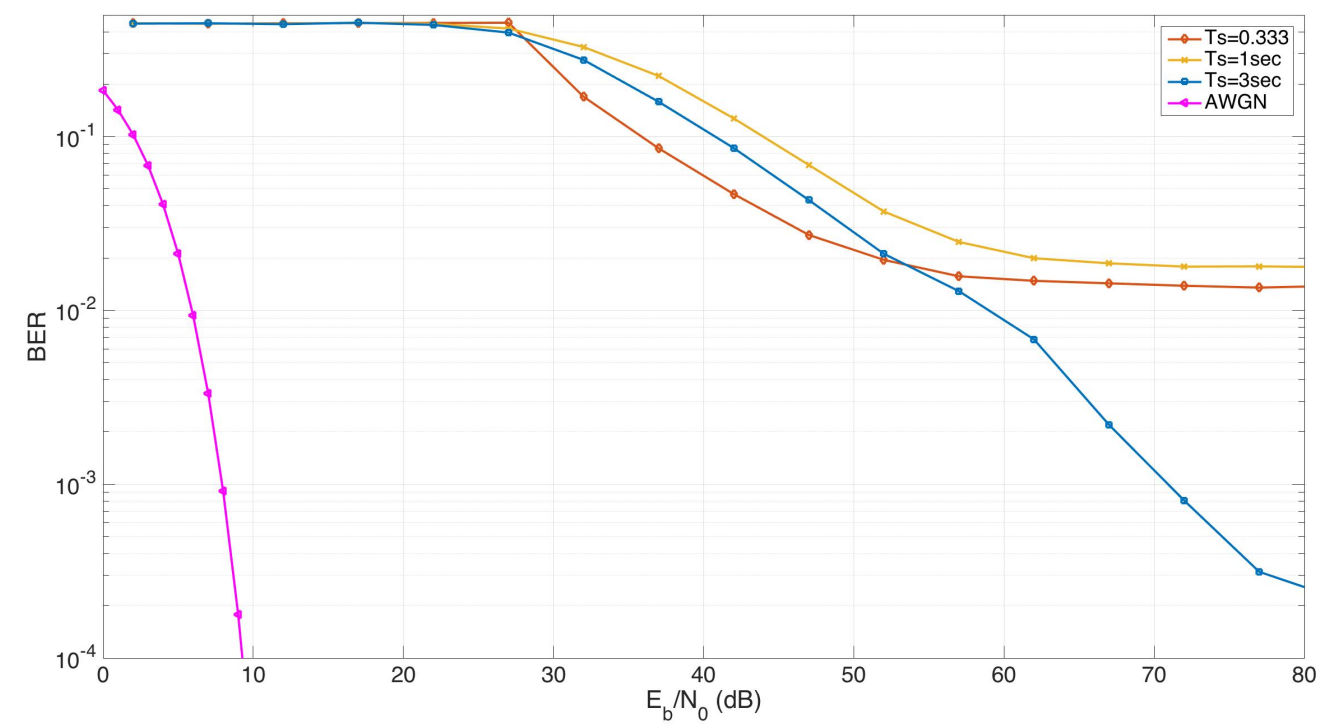

Figure 40: BPSK with different symbol times over a range of $5 \mathrm{~km}$ and $20 \%$ of ice cover. 


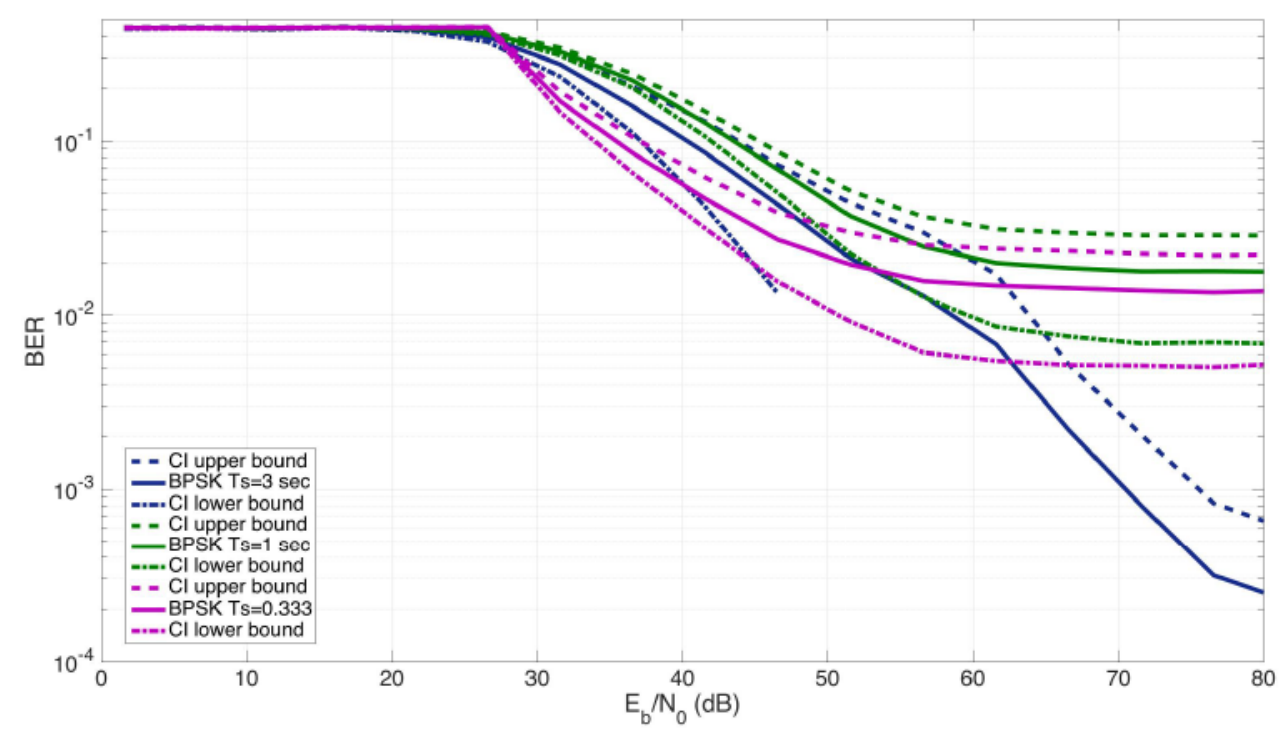

Figure 41: Confidence interval with 90\% confidence level. BPSK with $20 \%$ of ice cover in $5 \mathrm{~km}$ range. The Figure shows three different symbol times used.

In Figure 40, one can see that the BER drops below $10{ }^{2}$ when we have $E_{b} / N_{0}$ greater than $55 \mathrm{~dB}$ and symbol time equals three seconds. The performance gets much worse as the symbol time is decreased or in other words when we increase the data rate. This is also shown in Figure 41. 


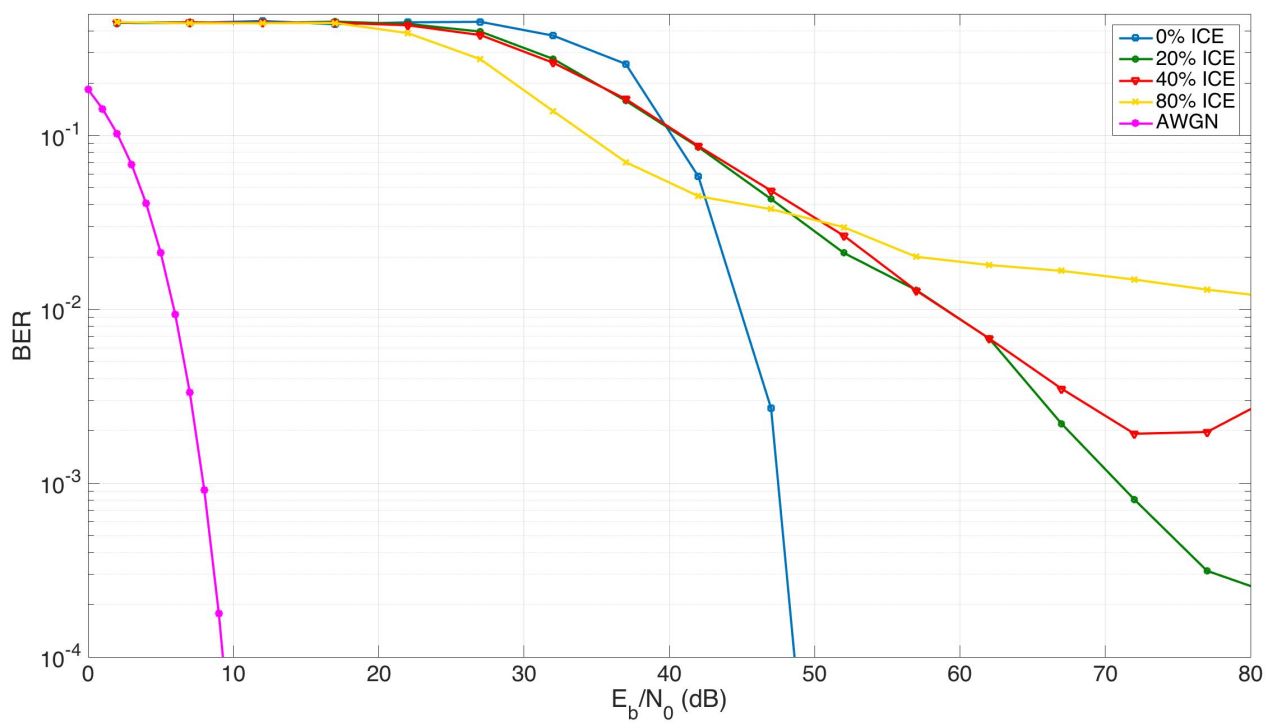

Figure 42: BPSK with different ice cover percentage over a range of $5 \mathrm{~km}$ using a symbol time of three seconds.

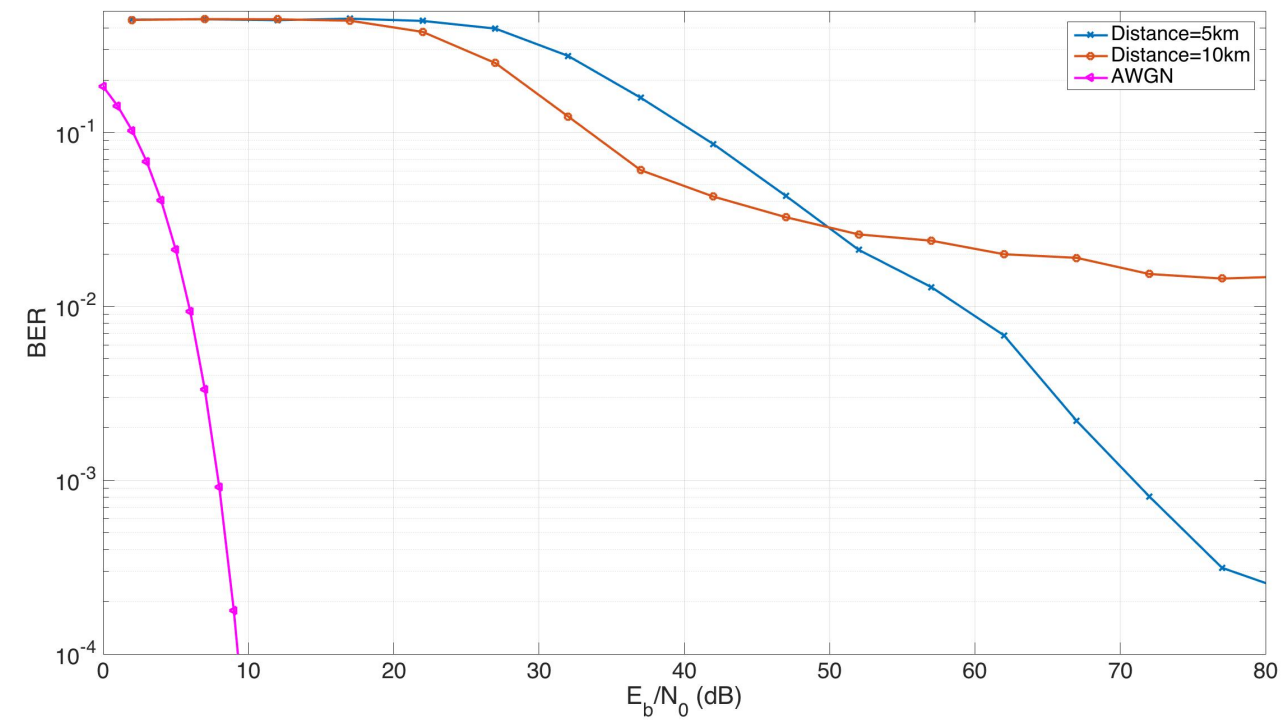

Figure 43: BPSK with 20\% of ice cover but two different ranges and same symbol time of three seconds 


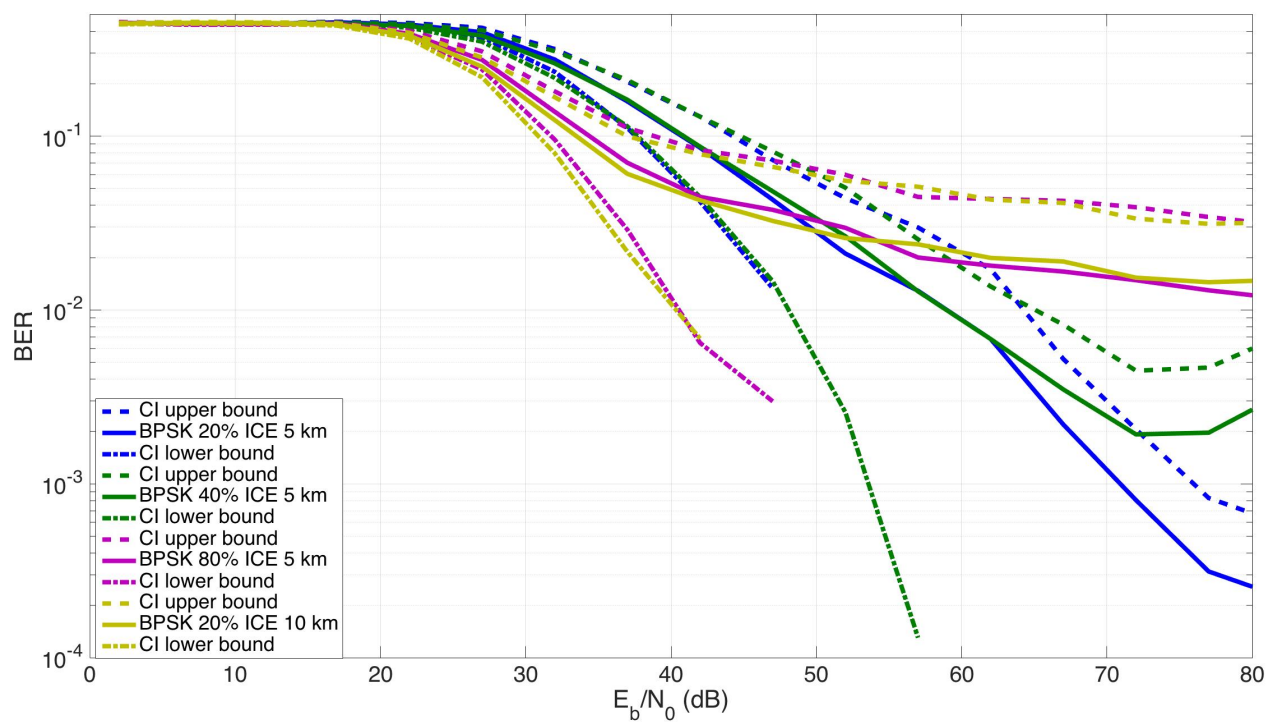

Figure 44: Confidence interval with $90 \%$ confidence level. BPSK with $20 \%, 40 \%, 80 \%$ of ice cover in $5 \mathrm{~km}$ range and $20 \%$ ice cover in $10 \mathrm{~km}$ range. Symbol time is equals to three seconds.

Using a symbol time of three seconds, we calculated the BER for four environments each with different ice cover percentages. We used 30 different impulse responses for each of the ice cover percentages. We calculated the mean BER of these files. The average calculated and shown in Figures 42 and 43 shows that the performance degrades with more ice cover or when the range is increased without adjusting the symbol time. However, the confidence intervals plot in Figure 44 shows that the upper bound of the confidence interval increases with more ice cover or longer range but the lower bound is similar in all cases. 


\subsubsection{Frequency-Shift Keying Performance}

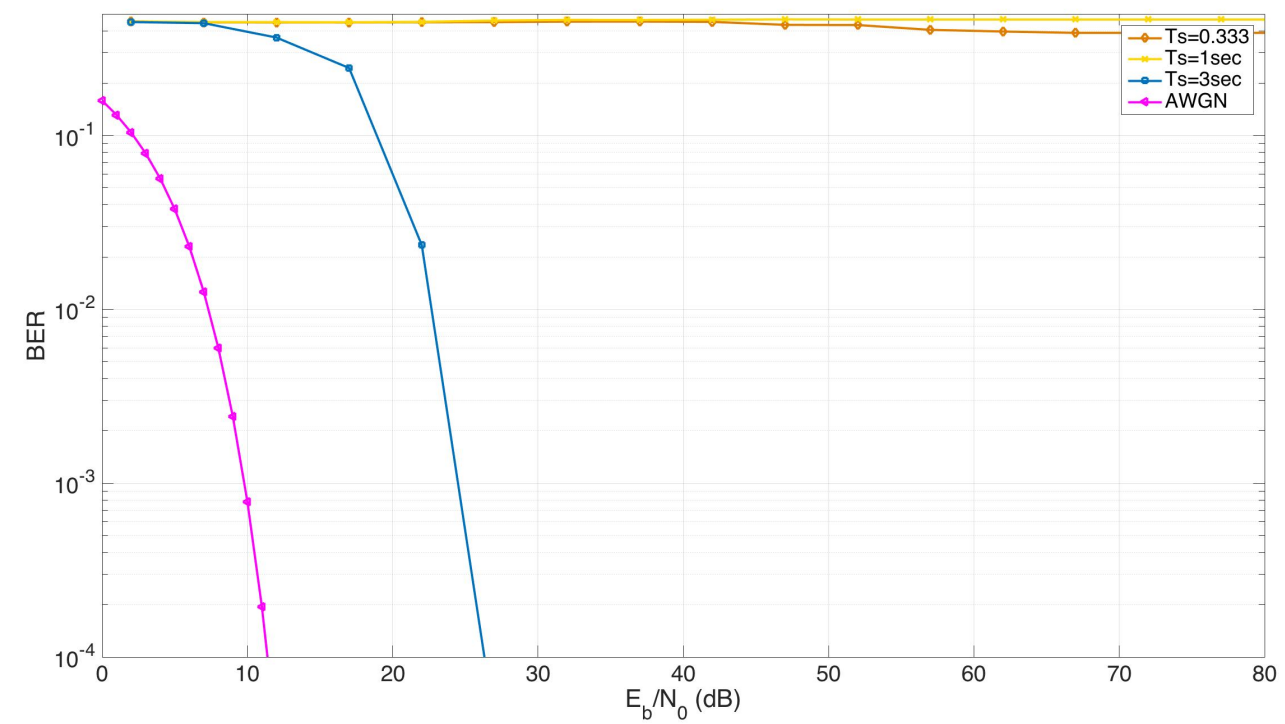

Figure 45: 2-FSK with different symbol times over a range of $5 \mathrm{~km}$ and $20 \%$ of ice cover.

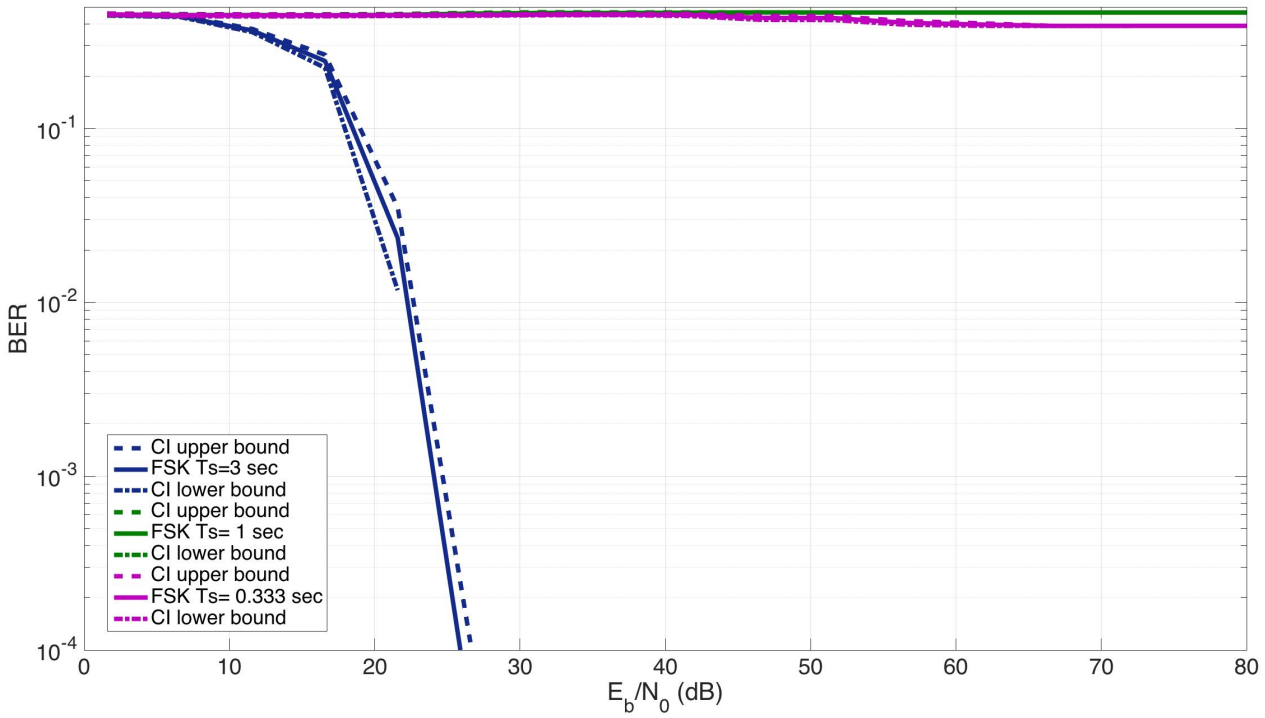

Figure 46: Confidence interval with $90 \%$ confidence level. 2-FSK with $20 \%$ of ice cover in $5 \mathrm{~km}$ range. The Figure shows three different symbol times used. 
Figure 45 shows the results of the FSK simulations over a range of $5 \mathrm{~km}$ and $20 \%$ of ice cover. It shows that only when the symbol time is much greater than the delay spread, it is possible to establish reliable communications. Otherwise, when the symbol time is one or one third of second the BER is $50 \%$. For equal performance, FSK requires a much lower $E_{b} / N_{0}$ ratio than BPSK. Therefore, the FSK performance is better at low bit rates in the conditions we have simulated. Figure 46 shows the confidence intervals plot of Figure 45.

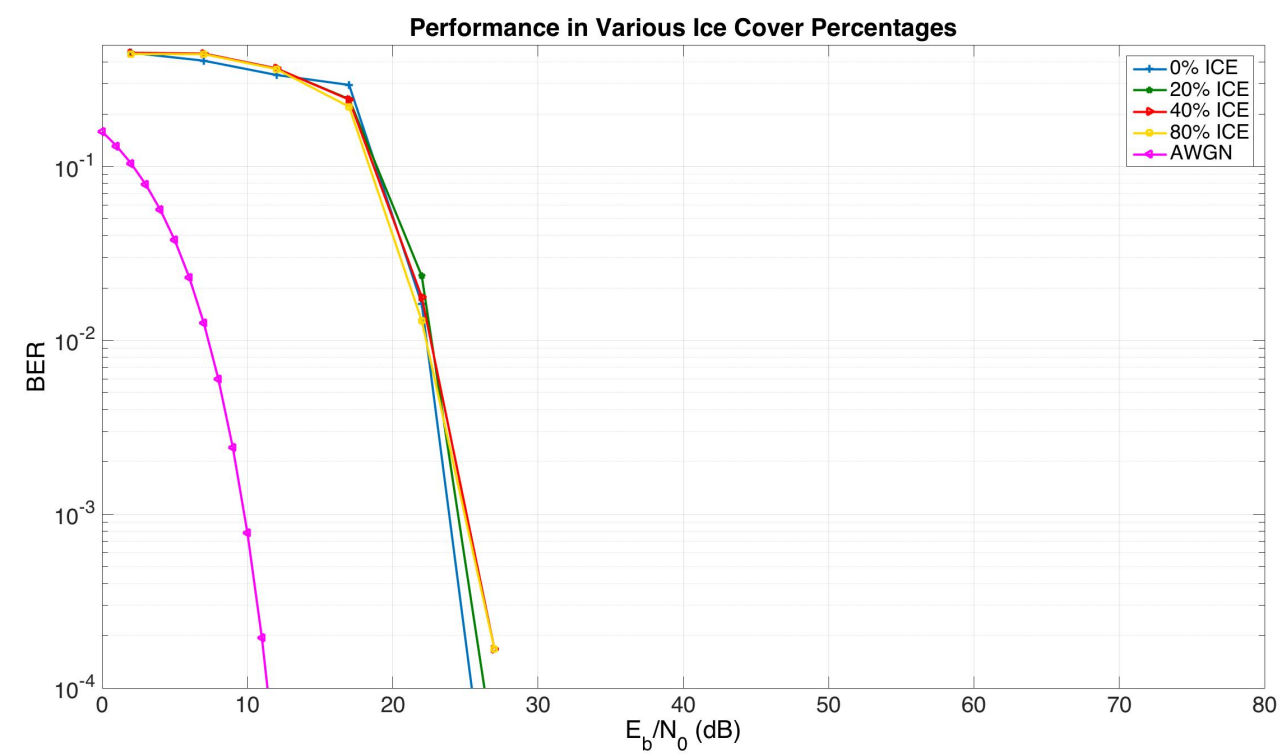

Figure 47: 2-FSK with different ice cover percentages over a range of $5 \mathrm{~km}$ using a symbol time of three seconds.

Figure 47 show the results of the simulation of FSK over several ice cover percentages shows almost no variations in the performance. The ice cover percentages simulated are $0 \%, 20 \%, 40 \%$ and $80 \%$ using only a symbol time of three seconds. In the conditions we have simulated, we conclude that FSK is not sensitive to ice cover. Figure 48 shows that when the range is increased from $5 \mathrm{~km}$ to $10 \mathrm{~km}$, FSK performance is almost similar. In the conditions and ranges we have simulated, we conclude that there is no variation in the performance between the two ranges simulated. 


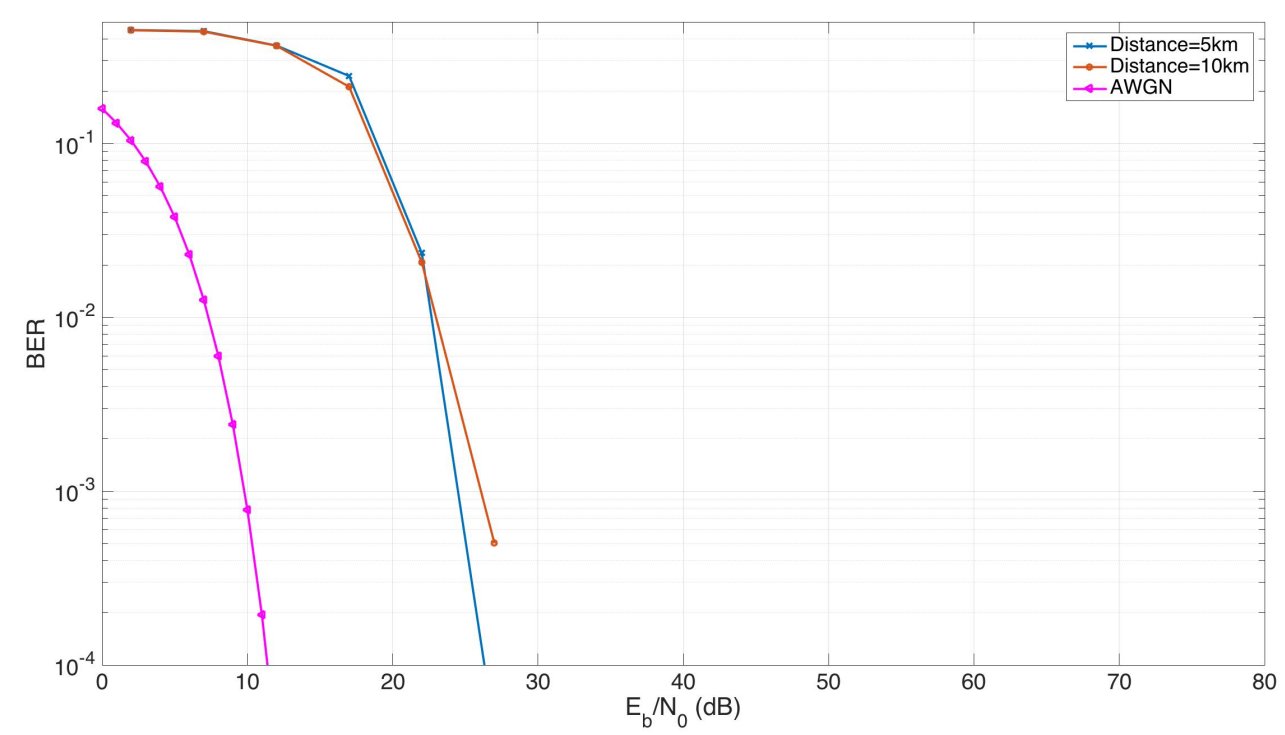

Figure 48: 2-FSK with $20 \%$ of ice cover, two different ranges and same symbol time of three seconds.

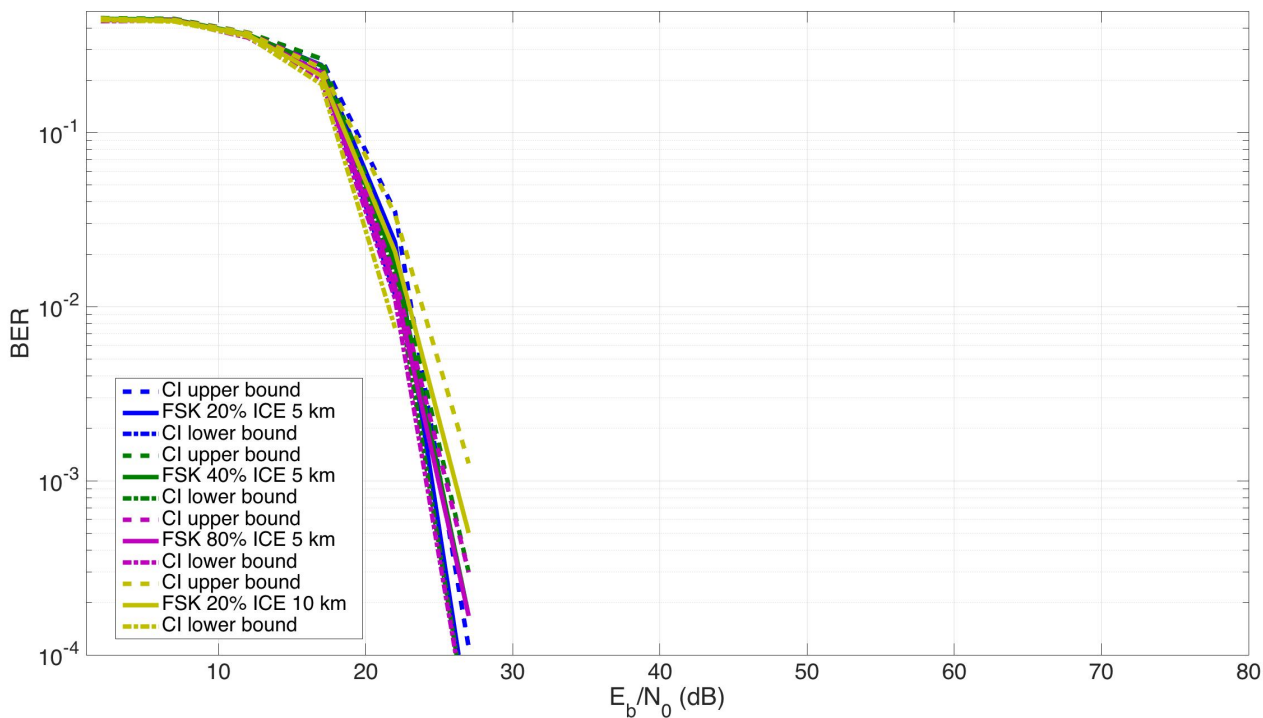

Figure 49: Confidence interval with 90\% confidence level. 2-FSK with 20\%, 40\%, $80 \%$ of ice cover in $5 \mathrm{~km}$ range and $20 \%$ ice cover in $10 \mathrm{~km}$ range. Symbol time is equals to three seconds. 


\subsubsection{Frequency Hopping-Continuous Phase-Shift Keying Performance}

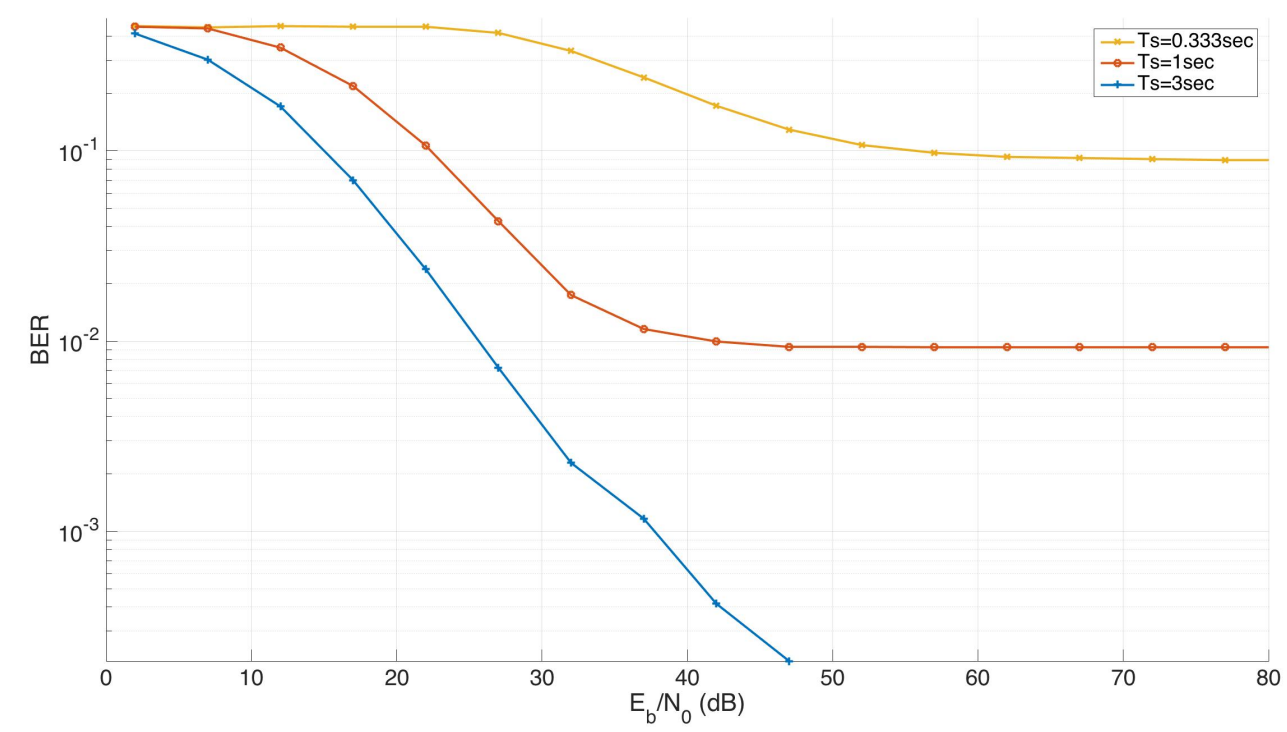

Figure 50: FH-CPFSK with different symbol times over a range of $5 \mathrm{~km}$.

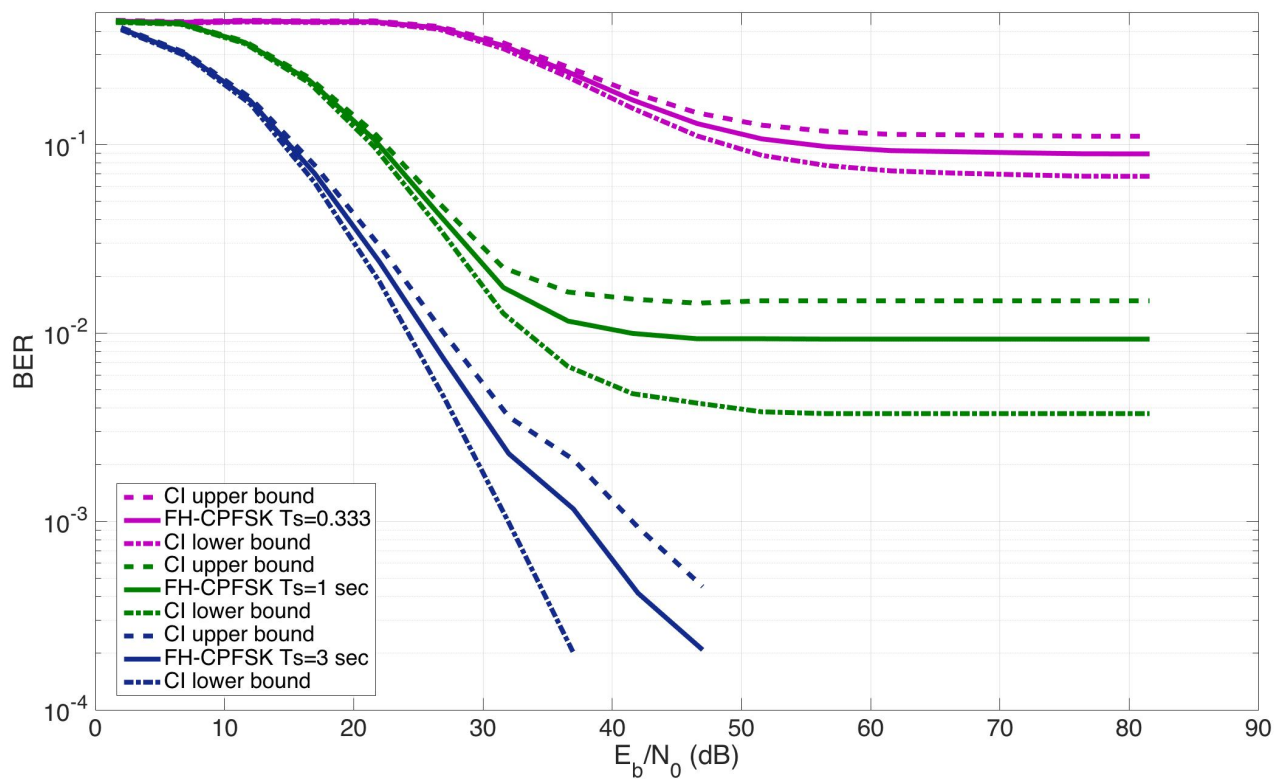

Figure 51: Confidence interval with $90 \%$ confidence level. FH-CPFSK with $20 \%$ of ice cover in $5 \mathrm{~km}$ range. The Figure shows three different symbol times used.

Figure 50 shows the performance of Frequency Hopping CPFSK with three different 
symbol times. The symbol times are three, one and one third of a second. The hopping rate is one hop per symbol. We use 16 different frequencies. Figure 51 shows the corresponding confidence interval BER plots.

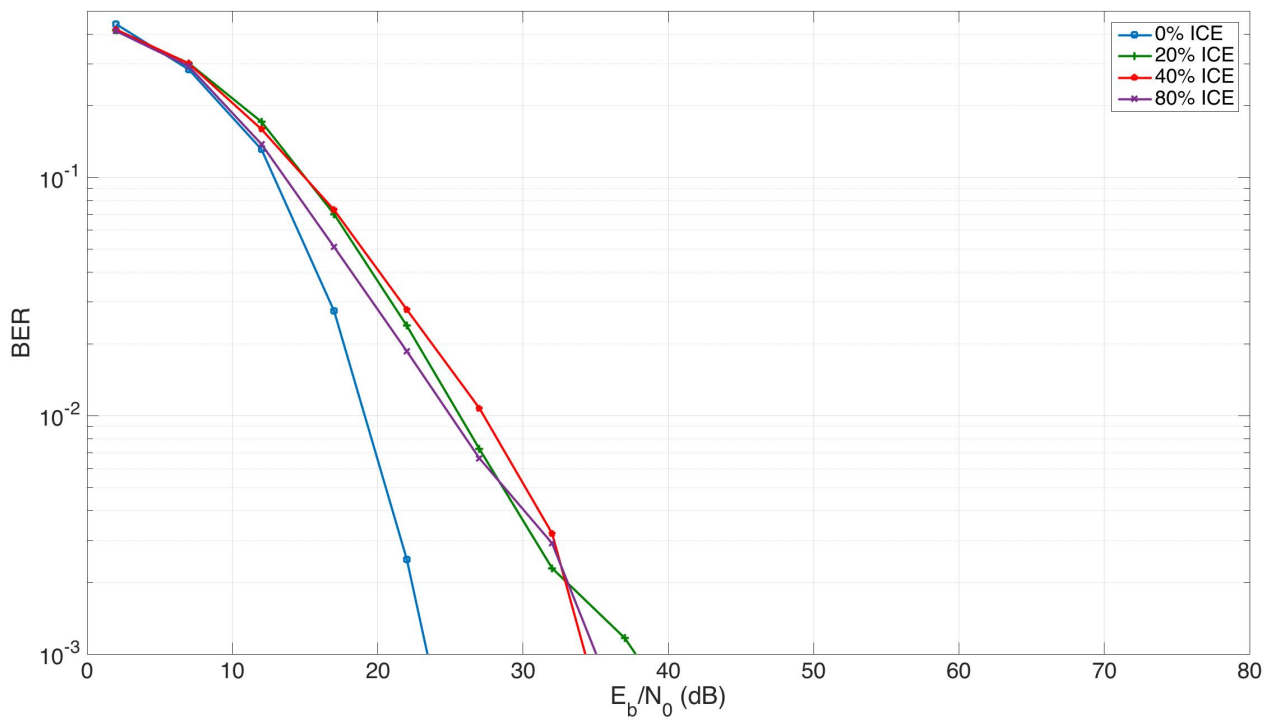

Figure 52: FH-CPFSK with different ice cover percentages over a range of $5 \mathrm{~km}$ using a symbol time of three seconds.

In presence of ice, the simulation of different ice cover percentages shows a little variations of the performance of FH-CPFSK. It performs best in the absence of ice. The result of increasing the range without changing the symbol time is shown in Figure 53. There is a small variation in the performance. Their corresponding conficence interval plots are shown in Figure 54. 


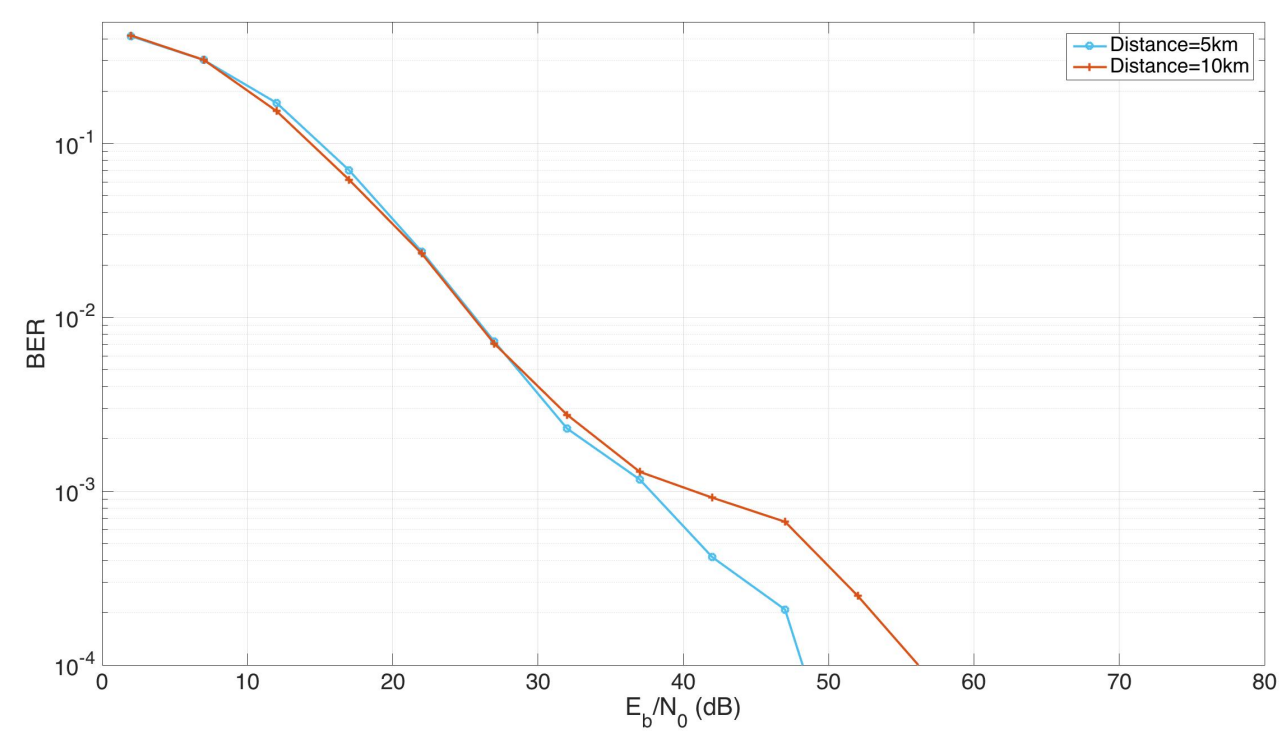

Figure 53: FH-CPFSK with $20 \%$ of ice cover, two different ranges and symbol time of three seconds.

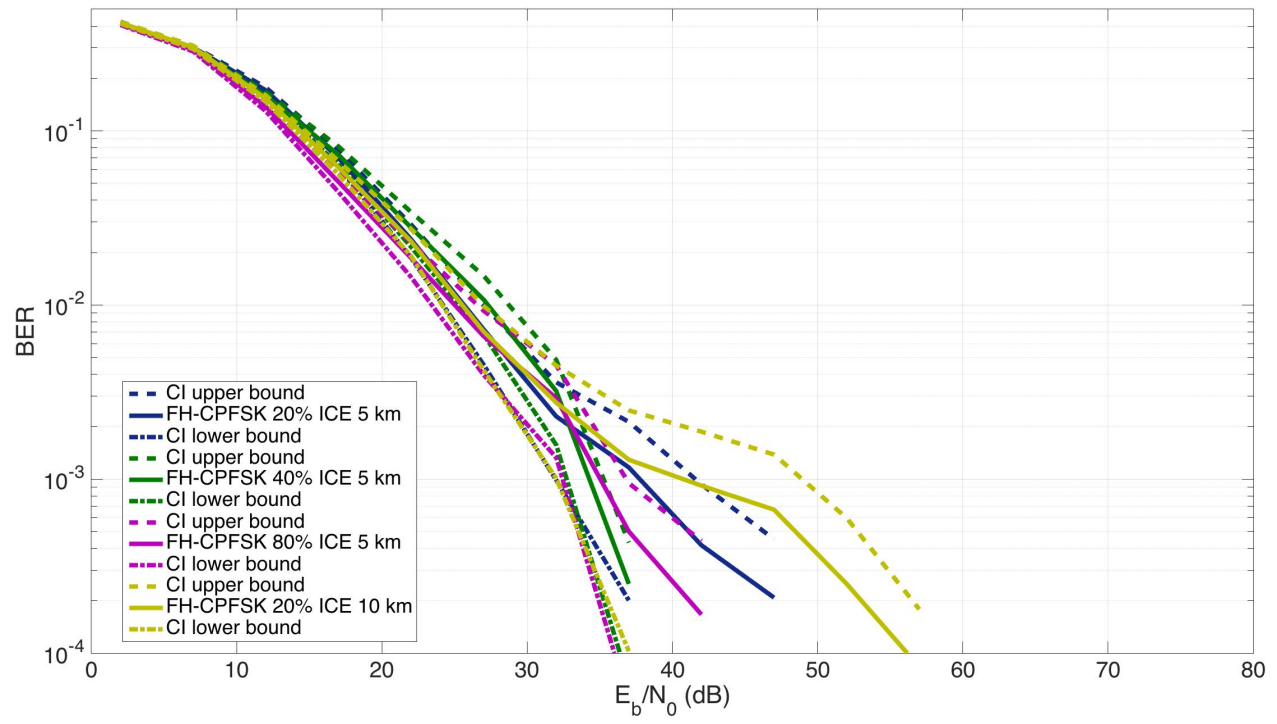

Figure 54: Confidence interval with 90\% confidence level. FH-CPFSK with 20\%, $40 \%, 80 \%$ of ice cover in $5 \mathrm{~km}$ range and $20 \%$ ice cover in $10 \mathrm{~km}$ range. Symbol time is equals to three seconds. 


\subsubsection{Orthogonal Frequency-Division Multiplexing Performance}

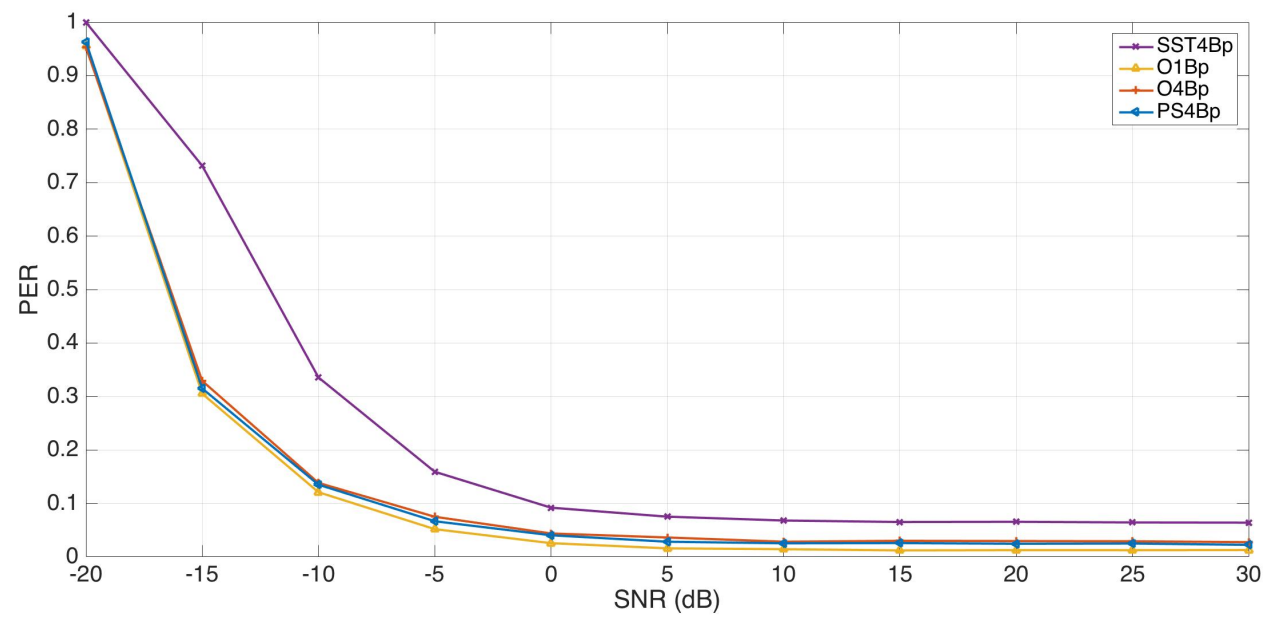

Figure 55: OFDM with different packet sizes and symbol times over a range of $5 \mathrm{~km}$.

In Figure 55, the label O4Bp refers to an OFDM with a payload size of four bytes. While the label O1Bp refers to an OFDM with a payload size of one byte. However, this produces more overhead. The label PS4Bp indicates an OFDM signal where pulse shaping is applied to each OFDM symbol. The label SST4Bp is associated with an OFDM symbol time $\left(T_{s}\right)$ equals to 1.333 seconds, which is a higher data rate OFDM. For OFDM, we calculate the Packet Error Rate (PER) vs. SNR, since the demodulator is based on certain synchronization and detection techniques, in which a whole frame might not be detected and lost. It is appropriate to use PER rather than BER. For all setups, we use 64 subcarriers, 48 of which carry data, 12 of the subcarriers are null. The remaining 4 are pilot subcarriers. The symbol time is three seconds for all the aforementioned setups except for the SST4Bp where it is 1.333 seconds. The modulation used is QPSK for the payload and BPSK for the header bits. The data rates are $2.2 \mathrm{bits} / \mathrm{sec}$ for both O3Bp and PS4Bp, $0.5 \mathrm{bit} / \mathrm{sec}$ for O1Bp and $4.8 \mathrm{bits} / \mathrm{sec}$ for SST4Bp. 


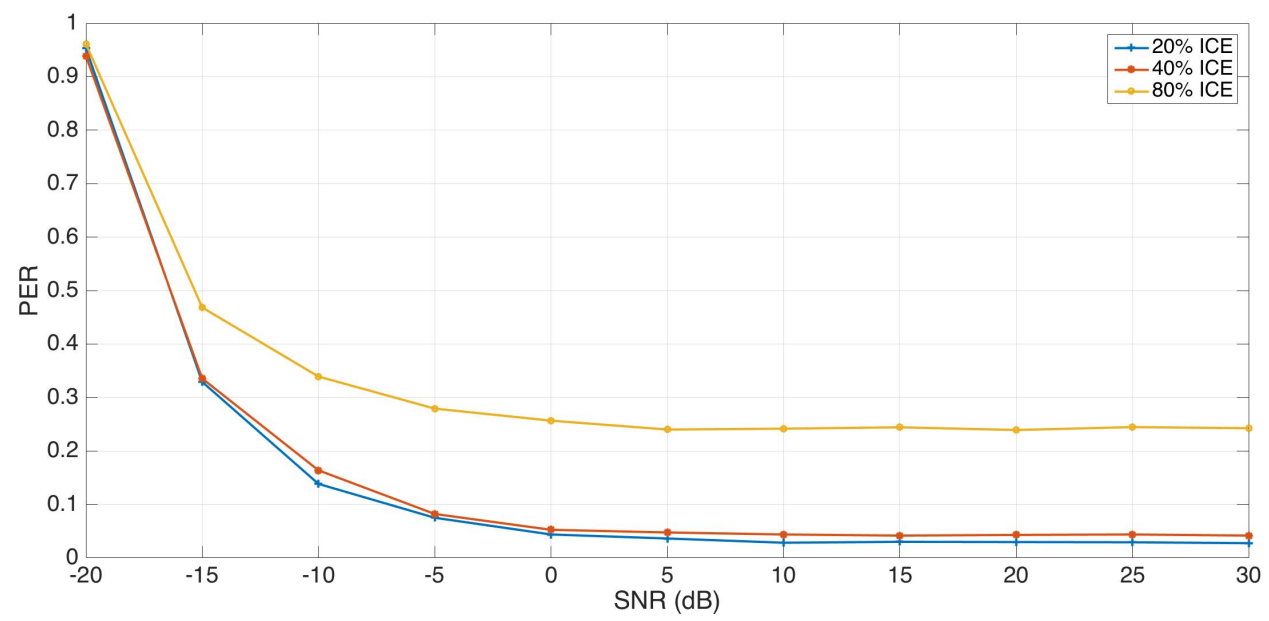

Figure 56: O4Bp OFDM with different ice cover percentages over a range of $5 \mathrm{~km}$ using a symbol time of three seconds.

The O4Bp OFDM performance over different ice cover percentages is depicted in Figure 56. OFDM is sensitive to ice cover percentages. At $80 \%$ ice cover, the PER converges to $30 \%$. Their CI Figures can be found in appendix A. 


\subsubsection{Comparison of the Performance of Various Modulation Techniques}

In Figures 57, 58, 60, 62 and 64, we compare the performance of BPSK vs. FSK vs. FH-CPFSK in different environments. All of the modulation techniques use a symbol time of three seconds and their center of frequency is $10 \mathrm{kHz}$.

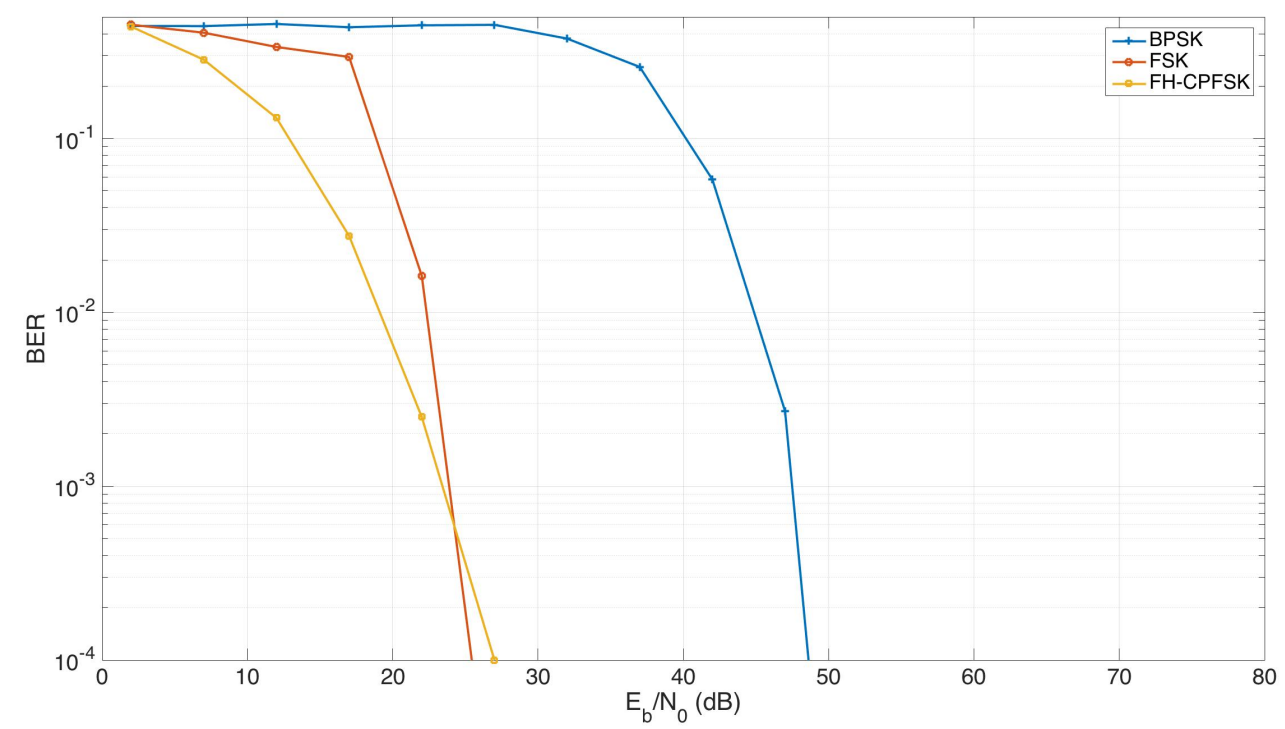

Figure 57: BPSK vs 2-FSK vs FH-CPFSK in $0 \%$ of ice cover over a range of $5 \mathrm{~km}$.

In the absence of ice, the simulation results show that FSK and FH-CPFSK have similar performance. However, they both outperform BPSK. 


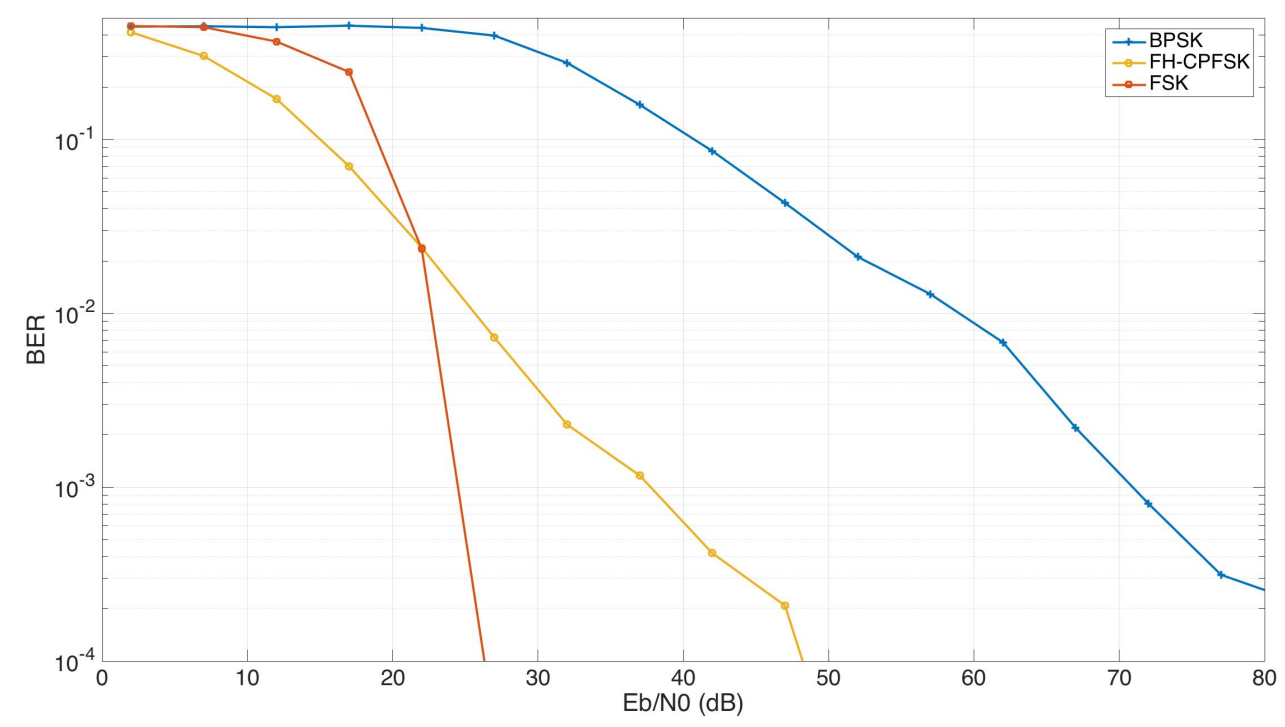

Figure 58: BPSK vs 2-FSK vs FH-CPFSK in $20 \%$ of ice cover over a range of $5 \mathrm{~km}$ environment.

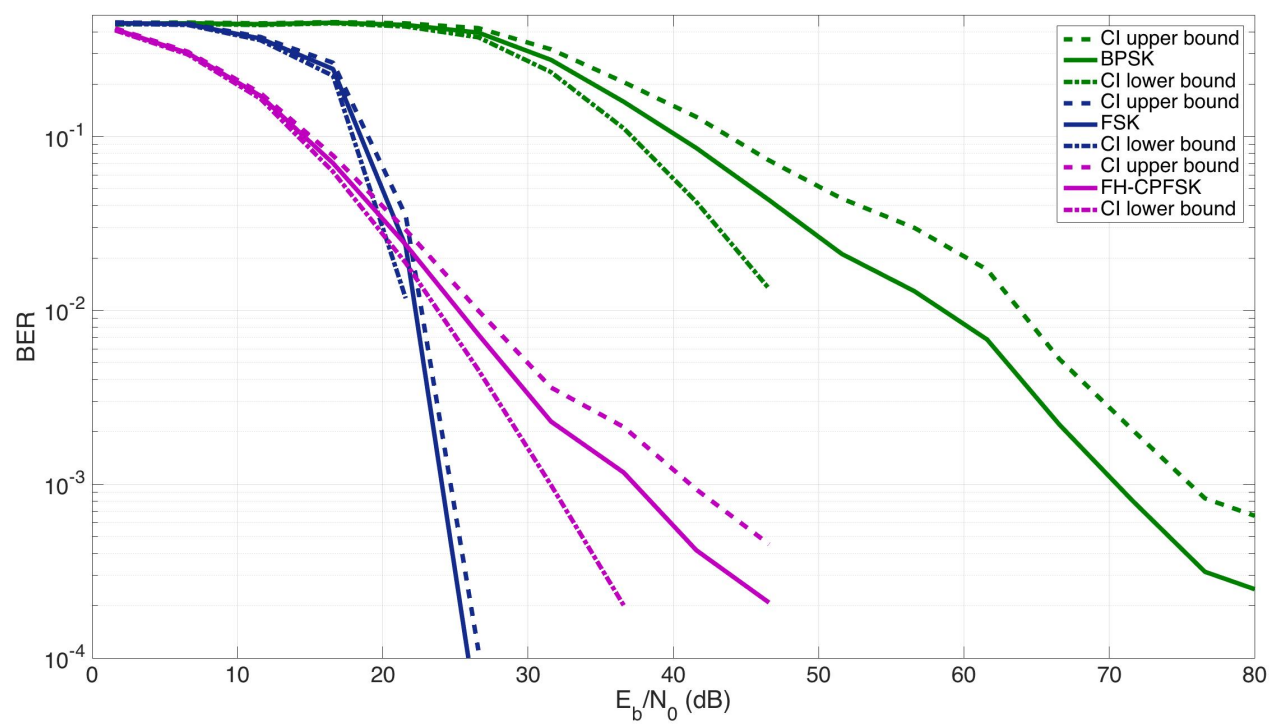

Figure 59: Confidence interval with 90\% confidence level. BPSK vs FSK vs FH-CFSK in $20 \%$ of ice cover over a range of $5 \mathrm{~km}$ environment.

The results shown in Figure 58 implies that the BPSK and FH-CPFSK performances are impacted by the existence of ice cover, unlike FSK which is not affected by ice cover. The corresponding confidence interval Figure is shown in Figure 59. 


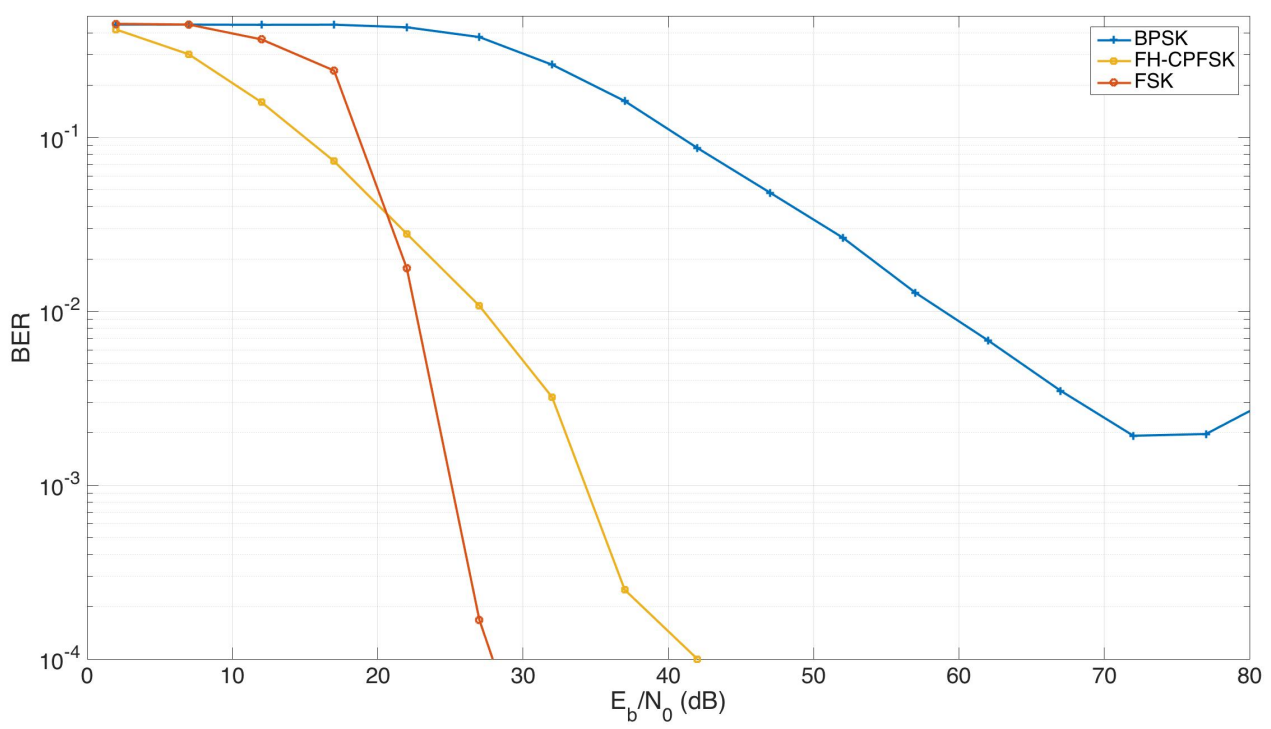

Figure 60: BPSK vs 2-FSK vs FH-CPFSK in $40 \%$ of ice cover over a range of $5 \mathrm{~km}$ environment.

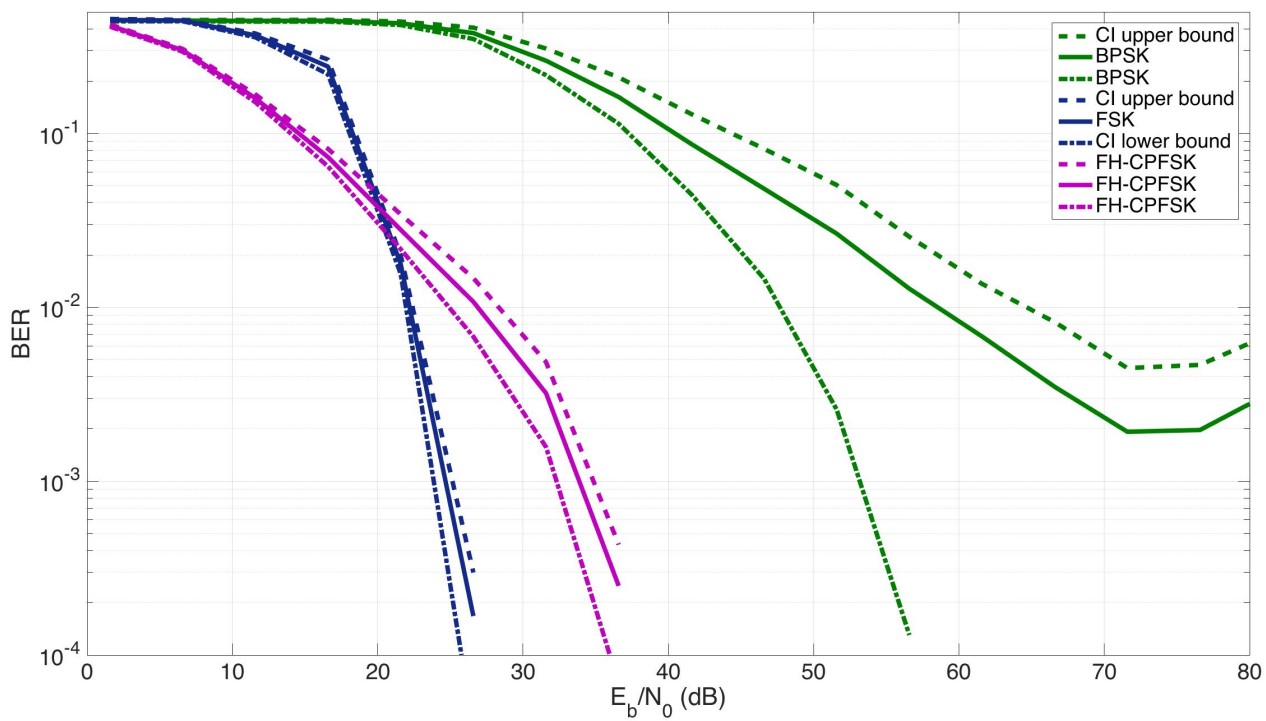

Figure 61: Confidence interval with 90\% confidence level. BPSK vs FSK vs FH-CFSK in $40 \%$ of ice cover over a range of $5 \mathrm{~km}$ environment.

Figure 60 implies that the FH-CPFSK performance is still about the same as when there is $20 \%$ of ice cover. However, the BPSK performance has degraded. The corresponding confidence interval Figure is shown in Figure 61. 


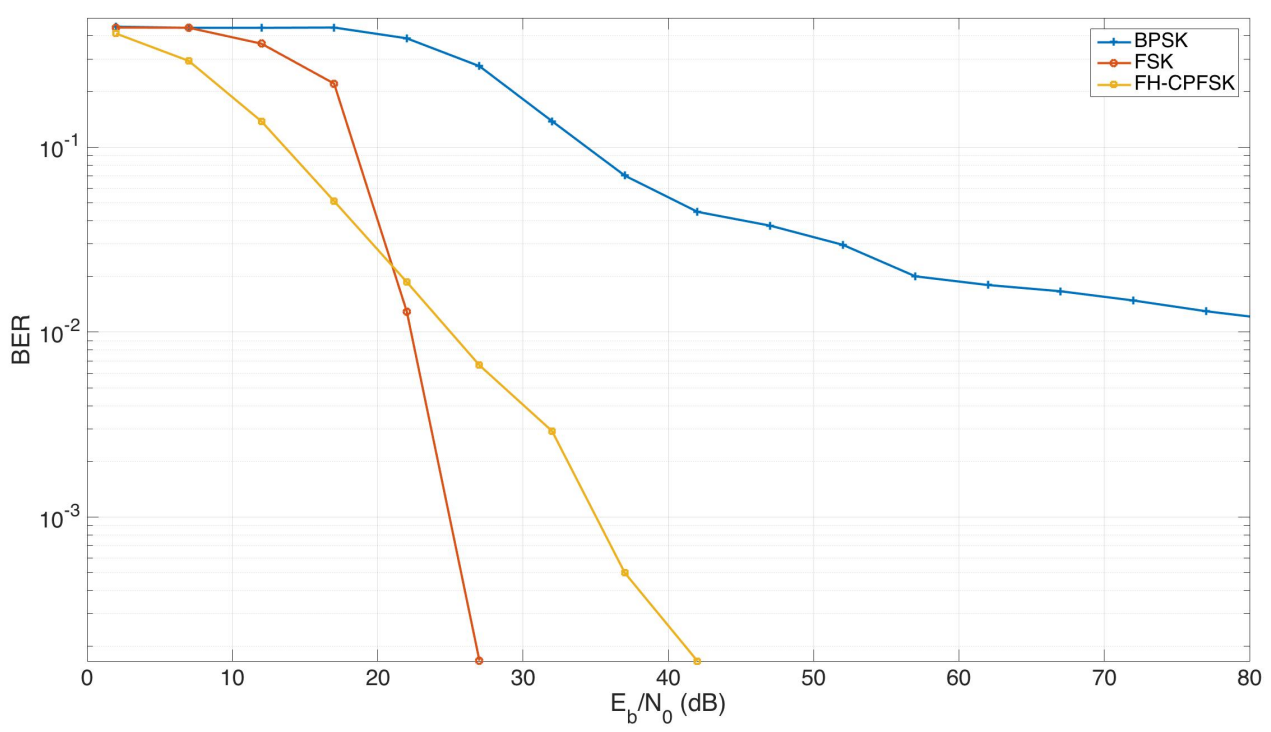

Figure 62: BPSK vs 2-FSK vs FH-CPFSK in $80 \%$ of ice cover over a range of $5 \mathrm{~km}$ environment.

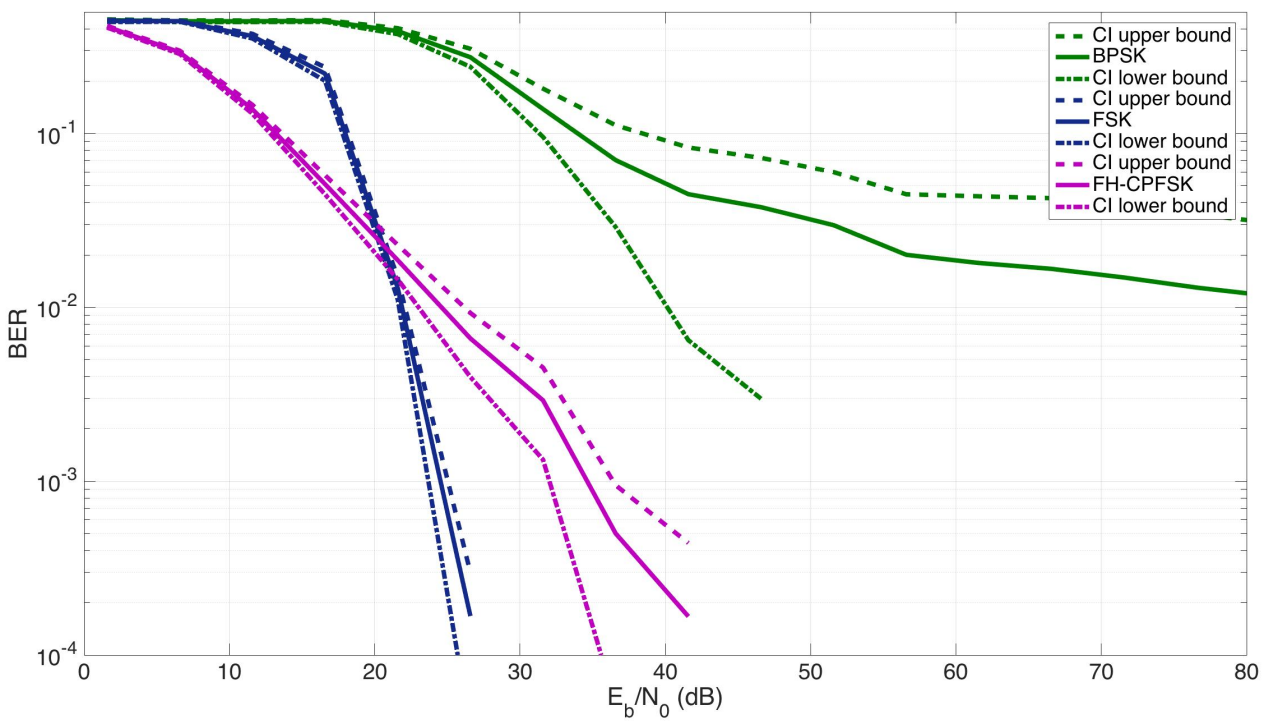

Figure 63: Confidence interval with 90\% confidence level. BPSK vs FSK vs FH-CFSK in $80 \%$ of ice cover over a range of $5 \mathrm{~km}$ environment.

The results in Figure 62 shows that a higher ice cover percentage does not affect the performance of FH-CPFSK or FSK. Their performances are almost invariant with 
ice covers $20 \%, 40 \%$ or $80 \%$. The corresponding confidence interval Figure is shown in Figure 63. While BPSK upper bound confidence interval did increase, the lower bound is about the same when the ice cover percentage is $20 \%$ or $80 \%$.

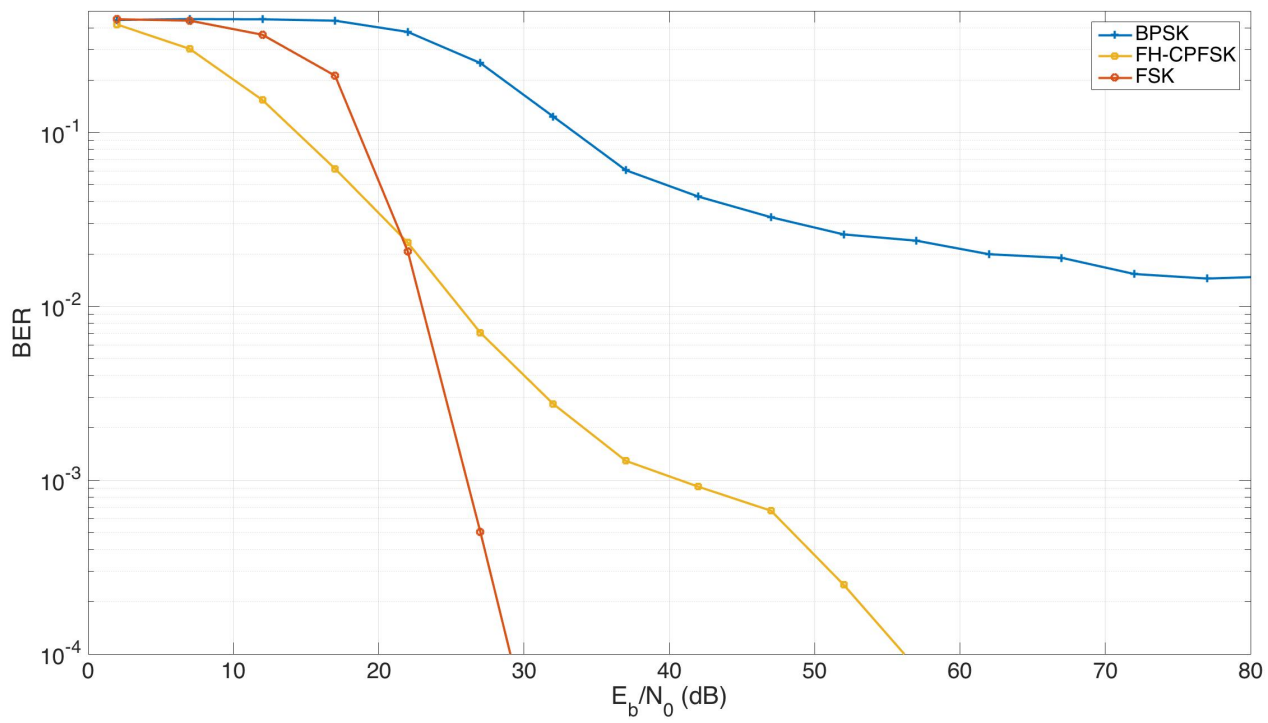

Figure 64: BPSK vs 2-FSK vs FH-CPFSK in 20\% of ice cover over a range of $10 \mathrm{~km}$ environment. 


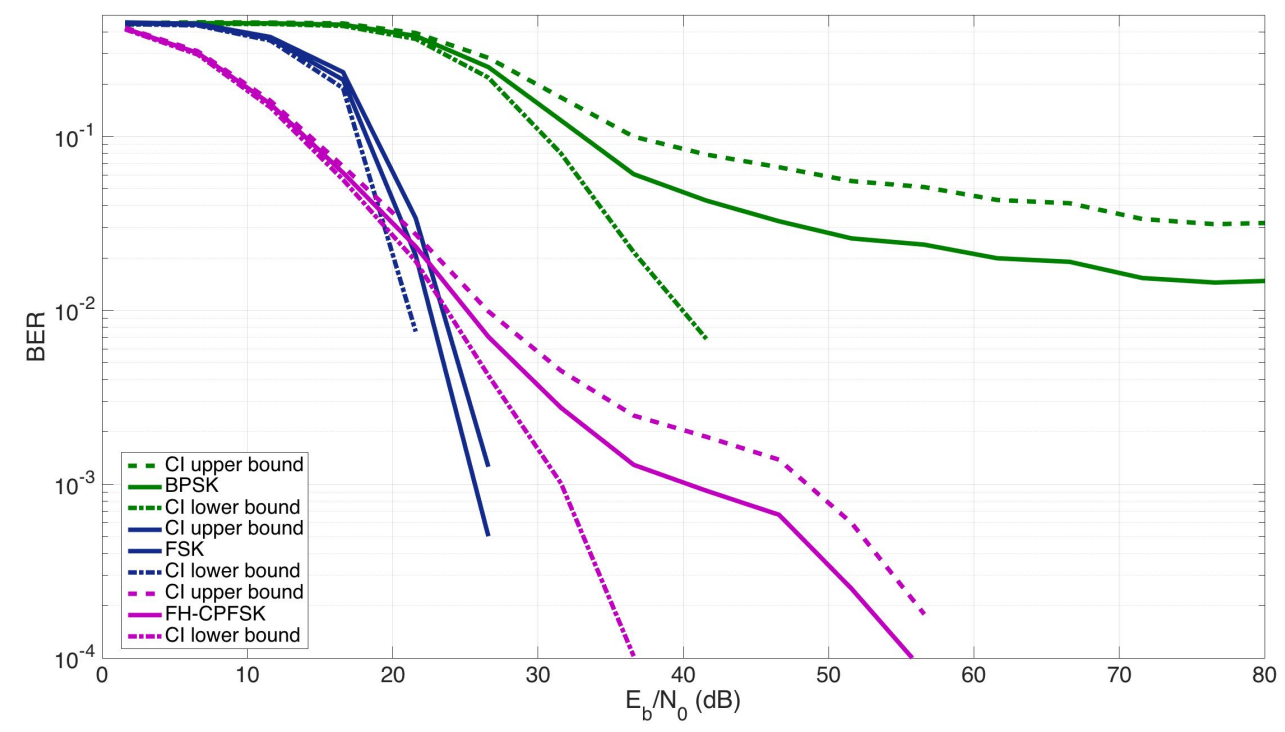

Figure 65: Confidence interval with 90\% confidence level. BPSK vs FSK vs FH-CFSK in $20 \%$ of ice cover over a range of $10 \mathrm{~km}$ environment. 
Figure 64 is a comparison of the performance of BPSK, FSK and FH-CPFSK for a $10 \mathrm{~km}$ range. The corresponding BER confidence interval figure plot is depicted in Figure 65.

In Figures 66, 67, 68, 69 and 70, we compare the PERs of BPSK vs FSK vs FHCPFSK vs O4Bp OFDM in different environments. Their corresponding confidence intervals can be found in appendix A. The packet size is four bytes. The center frequency for all modulation techniques and schemes is $10 \mathrm{kHz}$. All the modulation techniques use a symbol time of three seconds and their center of frequency is $10 \mathrm{kHz}$.

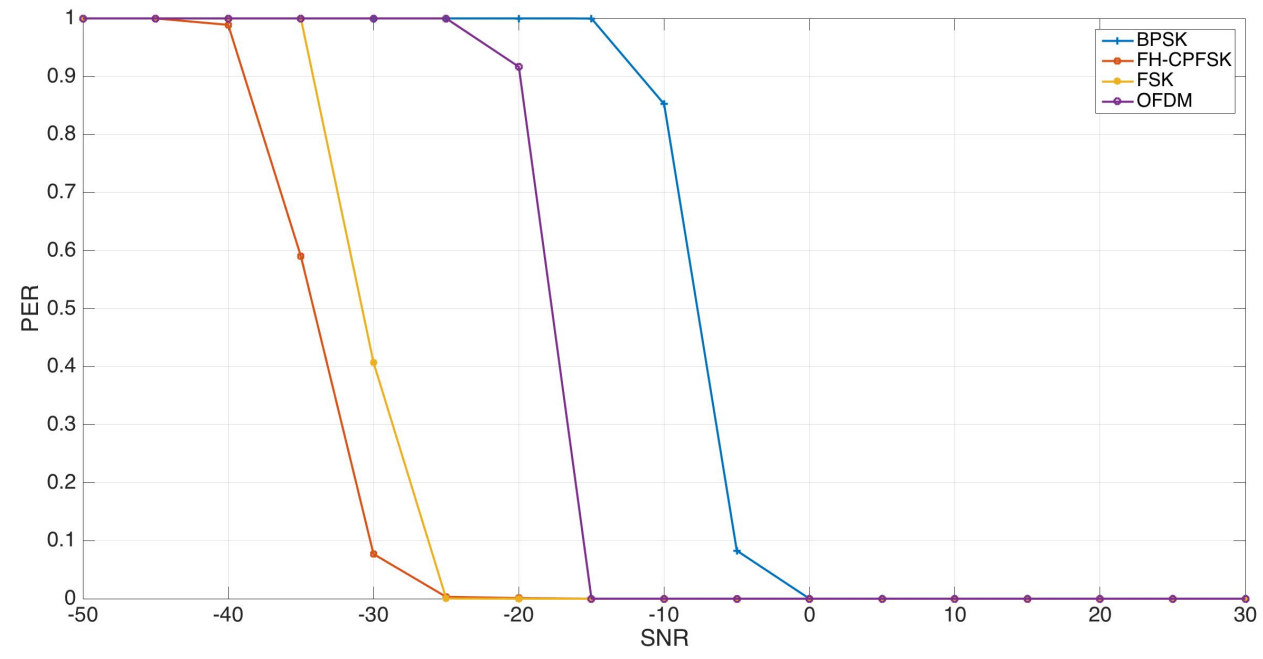

Figure 66: PER vs SNR of BPSK vs 2-FSK vs FH-CPFSK vs O4Bp OFDM in 0\% of ice cover over a range of $5 \mathrm{~km}$ environment.

In the absence of ice cover, we have observed that FH-CPFSK performs better than FSK, in the conditions we have tested, as shown in Figure 66. While O4Bp OFDM performance is better than BPSK performance, they both do worse than FSK and FH-CPFSK. 


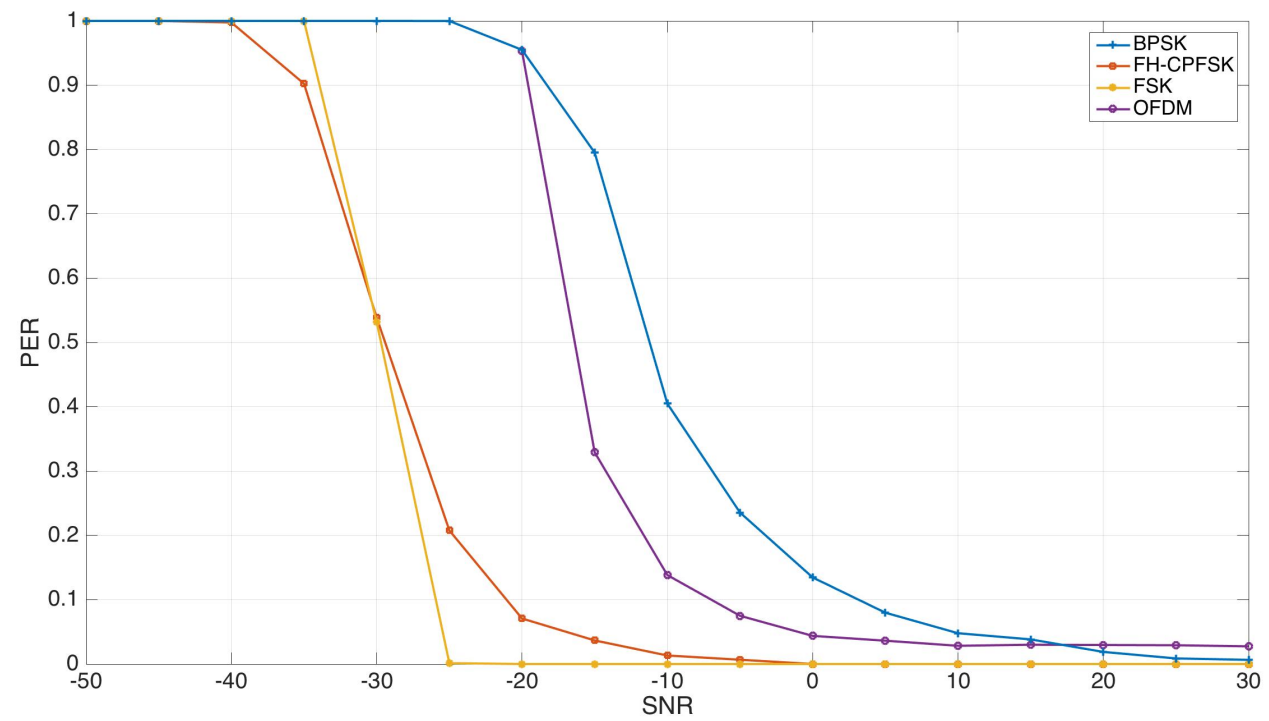

Figure 67: PER vs SNR of BPSK vs 2-FSK vs FH-CPFSK vs O4Bp OFDM in 20\% of ice cover over a range of $5 \mathrm{~km}$ environment.

In the condition where there is $20 \%$ of ice cover, the performance of FH-CPFSK is not better than FSK anymore. Both O4Bp OFDM and BPSK performances degrade in such a condition. The presence of ice has an effect on the performance of all of BPSK, FH-CPFSK and O4Bp OFDM. 


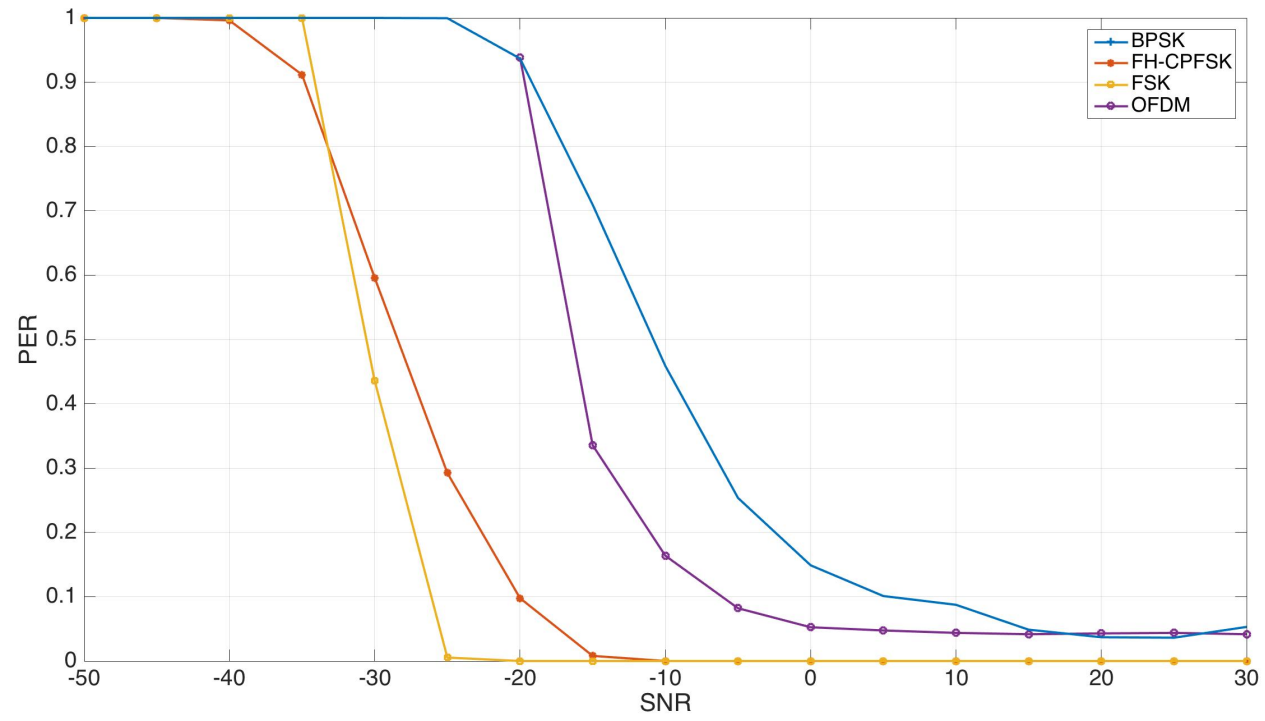

Figure 68: PER vs SNR of BPSK vs 2-FSK vs FH-CPFSK vs OFDM in $40 \%$ of ice cover over a range of $5 \mathrm{~km}$ environment.

With $40 \%$ ice cover, there is a slight degrade in the performance of O4Bp OFDM with respect to the $20 \%$ ice cover condition. While BPSK, FSK and FH-CPFSK performance stayed almost the same as shown in Figure 68. 


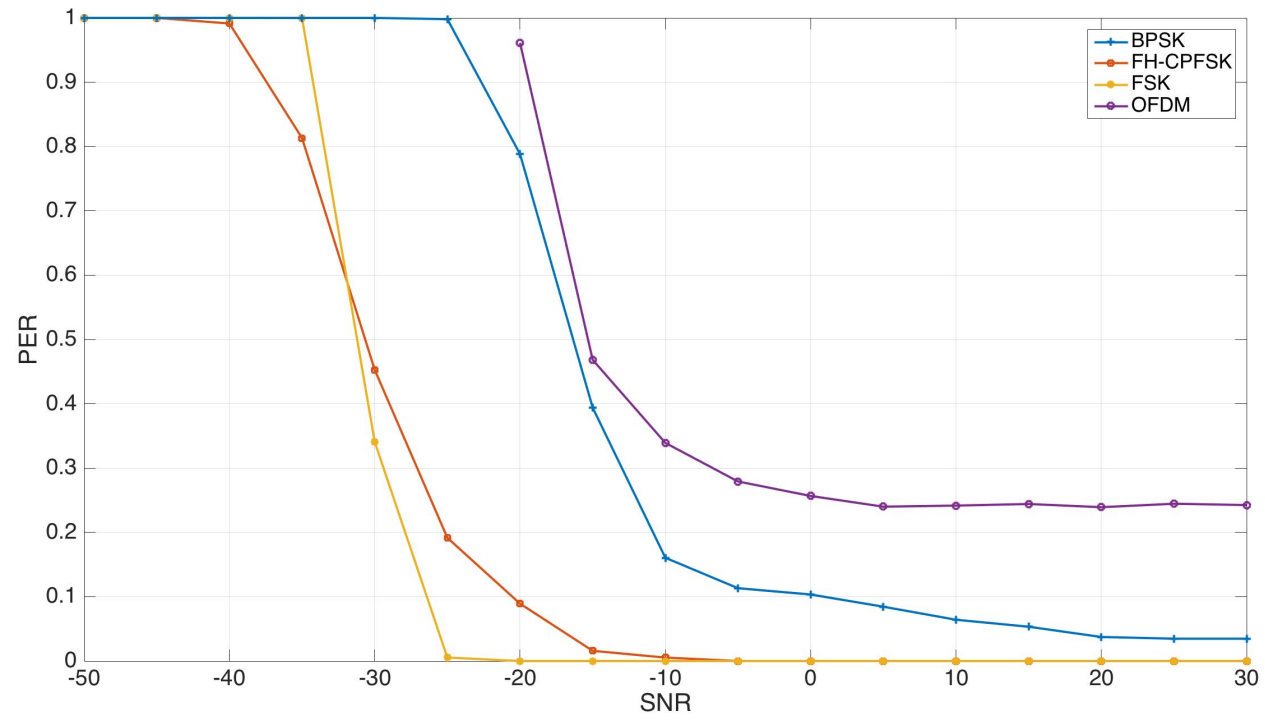

Figure 69: PER vs SNR of BPSK vs 2-FSK vs FH-CPFSK vs OFDM in $80 \%$ of ice cover over a range of $5 \mathrm{~km}$ environment.

The impact of $80 \%$ ice cover on FSK, BPSK, FH-CPFSK and O4Bp OFDM is depicted in Figure 69. The degrade in performance is significant for O4Bp OFDM. The performances of FSK and FH-CPFSK are almost the same as in the $40 \%$ ice cover condition. BPSK outperform O4Bp OFDM in this condition. 


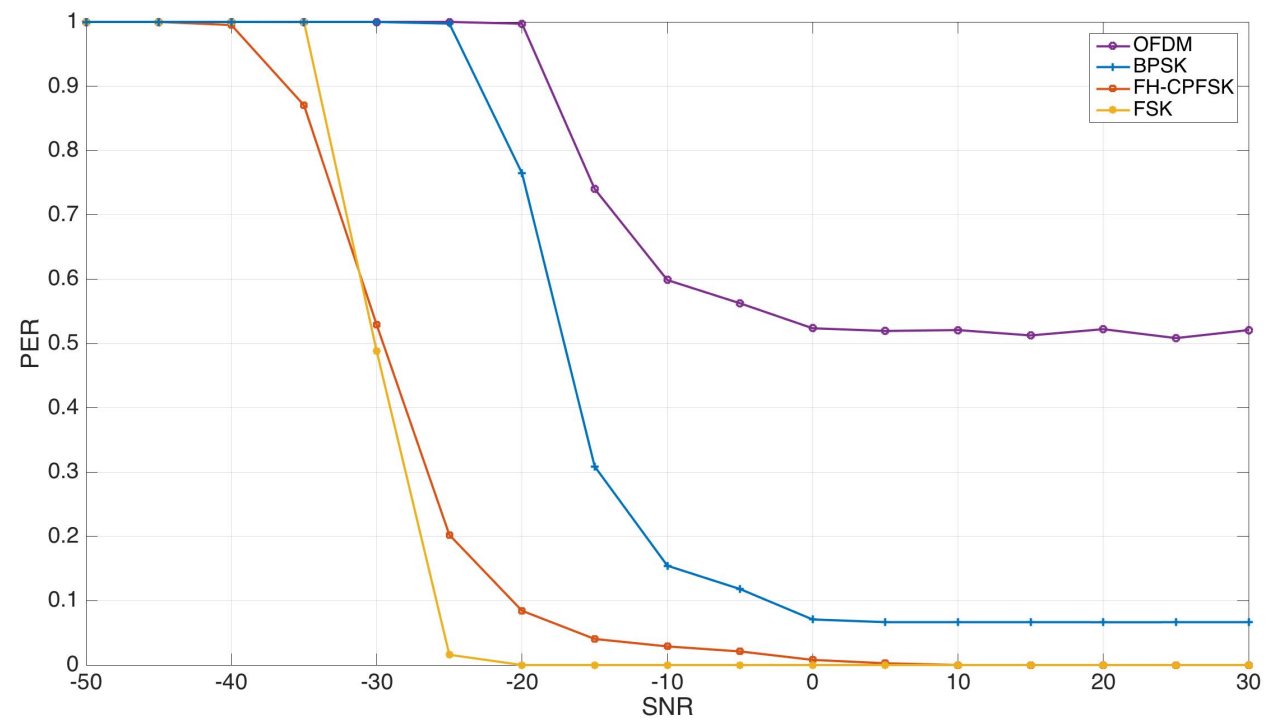

Figure 70: PER vs SNR of BPSK vs 2-FSK vs FH-CPFSK vs O4Bp OFDM in $20 \%$ of ice cover over a range of $10 \mathrm{~km}$ environment.

Figure 70 is a comparison of the performance of BPSK, FSK, FH-CPFSK and O4Bp OFDM for a $10 \mathrm{~km}$ range. The impact of a longer range, without changing the symbol time is drastic on O4Bp OFDM. It is less drastic on BPSK and FH-CPFSK and it is minor on FSK. BPSK outperforms O4Bp OFDM in this condition.

In the conditions we have simulated, Figures $66,67,68,69,70$ and their corrseponding confidence interval BER figures, show that overall FSK and FH-CPFSK perform better than OFDM and BPSK. OFDM is still better than BPSK in the conditions where the range is $5 \mathrm{~km}$ and ice cover percentages lower than $80 \%$. While in the conditions where the range is $5 \mathrm{~km}$ and there is $80 \%$ ice cover or when the range is $10 \mathrm{~km}$ with $20 \%$ ice cover BPSK outperform OFDM. Furthermore, FSK and FH-CPFSK performances has more resistance to ice cover. The case where FH-CPFSK performs better than FSK is when there is no ice cover. 


\subsection{Summary}

The results presented show that FSK and FH-CPFSK are both robust modulation techniques to be used in Arctic-like conditions. FSK performance is not impacted by the ice cover. With longer range, FSK symbol time need to be adjusted. FH-CPFSK performance under ice cover does not vary a lot when more ice cover percentage is present. However, with longer range and same symbol time, it is shown that FH-CPFSK performance is worse in comparison to FSK. FH-CPFSK and BPSK performs better than FSK when the symbol time was less than the delay spread. BPSK is affected by the presence of ice cover and range of receiver. Finally, OFDM performance is indeed much better than that of BPSK when less than $40 \%$ ice cover was present. However, OFDM performance degrades a lot when a longer distance was simulated without adjusting the symbol time or when more ice cover percentage was present. Its performance was worse than BPSK in an environment with $80 \%$ ice cover over $5 \mathrm{~km}$ or in an environment with $20 \%$ ice cover and a range of $10 \mathrm{~km}$. Table A.1 summarizes the simulated environments.

Table 5.1: Summary of Simulations.

\begin{tabular}{|l|l|l|l|l|l|}
\hline & $0 \%$ ice-5km & $20 \%$ ice-5km & $40 \%$ ice-5km & $80 \%$ ice-5km & $20 \%$ ice-10km \\
\hline BPSK & $\checkmark$ & $\checkmark$ & $\checkmark$ & $\checkmark$ & $\checkmark$ \\
\hline FSK & $\sqrt{ }$ & $\checkmark$ & $\checkmark$ & $\checkmark$ & $\checkmark$ \\
\hline FH-CPFSK & $\sqrt{ }$ & $\checkmark$ & $\checkmark$ & $\checkmark$ & $\checkmark$ \\
\hline OFDM & $\checkmark$ & $\checkmark$ & $\checkmark$ & $\checkmark$ & $\checkmark$ \\
\hline
\end{tabular}




\section{Chapter 6}

\section{Conclusion}

\subsection{Summary of Work}

The work in this thesis comprises the design and development of four modems using four different modulation schemes. The design and development of BPSK, FSK, FH-CPFSK and OFDM modems use GNU Radio existing modules and the work of David [40] for FH-CPFSK. In addition to the development of those modems, comes the simulations done using BELLHOP and MATLAB to mimic the effect of the arctic underwater communications conditions. The computation of the BER for different environments has been done. The effects of the ice cover on each of the modulation techniques used are analyzed with respect to the BER vs. $E_{b} / N_{0}$. Moreover, two different ranges are used to compare how does longer ranges affect the performance of each of the modulation schemes and transmission techniques. This work includes simulations of the arctic conditions and their impact on certain modulation schemes and transmission techniques. These simulations and results contribute to the knowledge of how ice cover and distance in Arctic-like environment affect the acoustic signals of the modulation schemes and transmission techniques used in this work. We have simulated $0 \%, 20 \%, 40 \%$ and $80 \%$ ice cover percentages for the range of $5 \mathrm{~km}$ and only $20 \%$ for the range of $10 \mathrm{~km}$. We have found that the performance OFDM is affected by ice cover. And with more ice cover percentage the performance of OFDM degrades. In the conditions we have tested, we have found that FSK is immune to the distortion caused by the ice cover. We have found that for longer ranges and without adjusting symbol time, BPSK and OFDM performances are also affected. Furthermore, FH-CPFSK performance was also affected by longer range but to a lesser extent than BPSK and OFDM. Finally, FSK was the least affected by the longer range. FH-CPFSK and BPSK performs better when the symbol time was less than the delay spread. However, when the symbol time is much greater than 
the delay spread, FSK outperform all other modulation schemes and transmission techniques at higher SNRs.

\subsection{Future Work}

The simulations done comprise stationary transmitters and receivers. Future work should include moving nodes. Moving nodes will cause Doppler spread. Doppler spread introduces significant interference. Moreover, advanced signal processing techniques including different training symbols and different detection techniques for OFDM, might be used to compensate for the phase distortion caused by the ice cover. Both FSK and FH-CPFSK have shown to have resistance against ice cover distortions, but Doppler spread needs to be compensated in order to achieve performance. Moreover, to compensate for the slow data rates, studies regarding the use of multicarrier techniques using FSK shall be conducted. Another fact to be considered is the frequency-dependent attentuation. In the work done in this thesis the attenuation considered is on the $10 \mathrm{kHz}$ center frequency, however FH-CPFSK uses $\pm 5625 \mathrm{kHz}$ from center frequency. Studies and simulations considering the effects of frequency-dependent attenuation on FH-CPFSK performance is required. An advanced approach would be the development of an adaptive system or even a cognitive system which could be implemented to overcome the complexities introduced by the underwater channel. A system that would change the modulation scheme or the symbol time according to the channel estimations done. A cognitive system is defined as a system that would be aware of its internal state and environment and make decisions about their operations by mapping that information against predefined objectives [24]. 


\section{Appendix A}

For the reproduction of the simulations refer to github directory [53].

The following Figures show the BER confidence intervals for BPSK simulations.

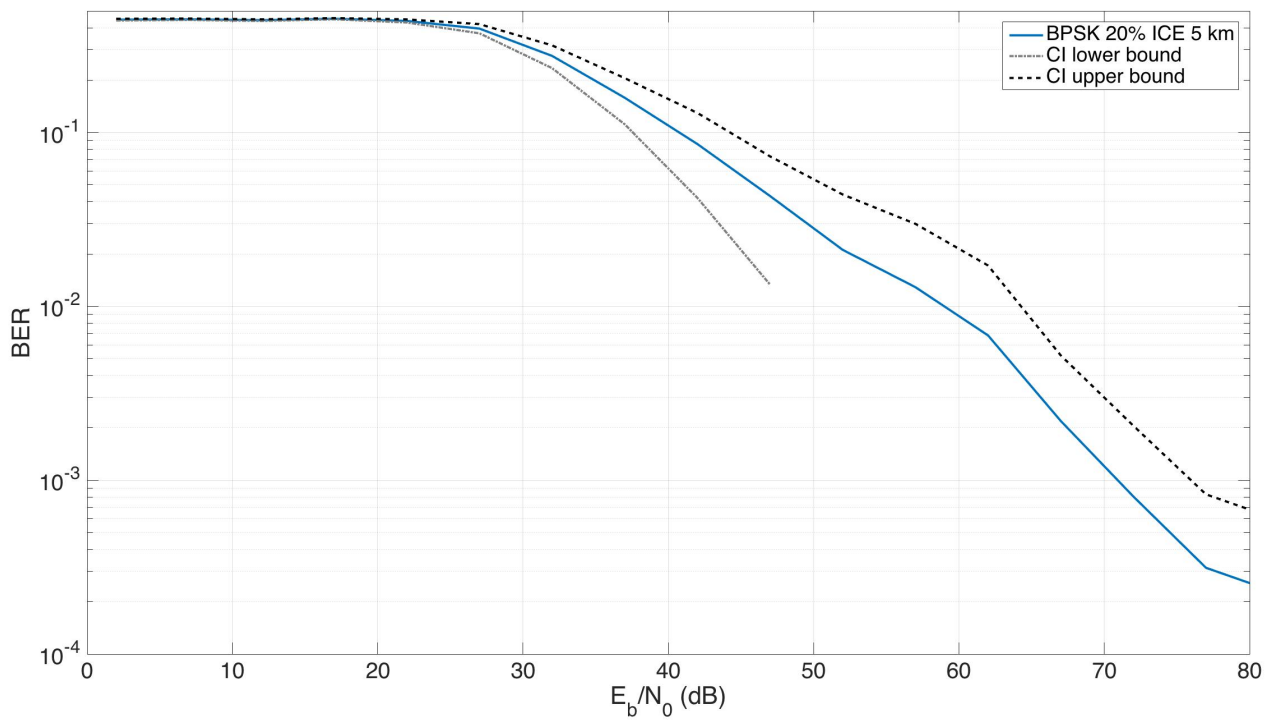

Figure 71: Confidence interval with 90\% confidence level. BPSK with $20 \%$ of ice cover, $5 \mathrm{~km}$ range and symbol time of three seconds. 


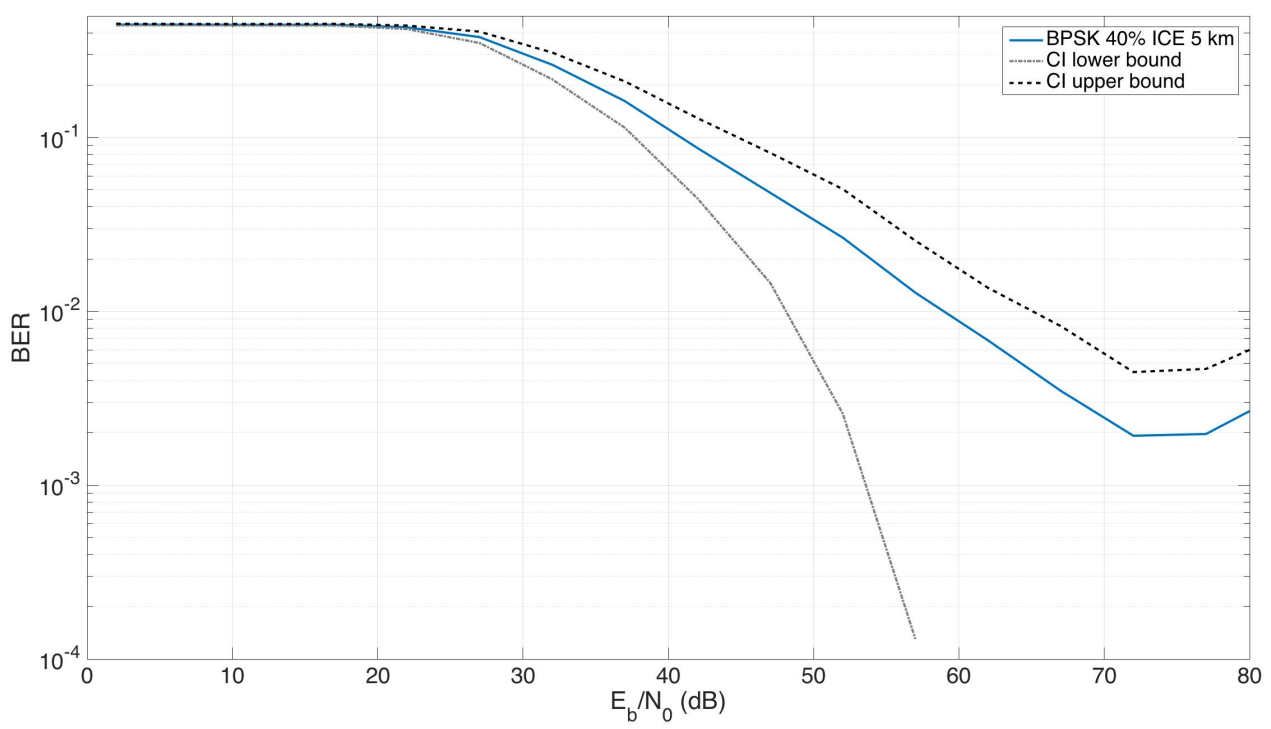

Figure 72: Confidence interval with 90\% confidence level. BPSK with $40 \%$ of ice cover, $5 \mathrm{~km}$ range and symbol time of three seconds.

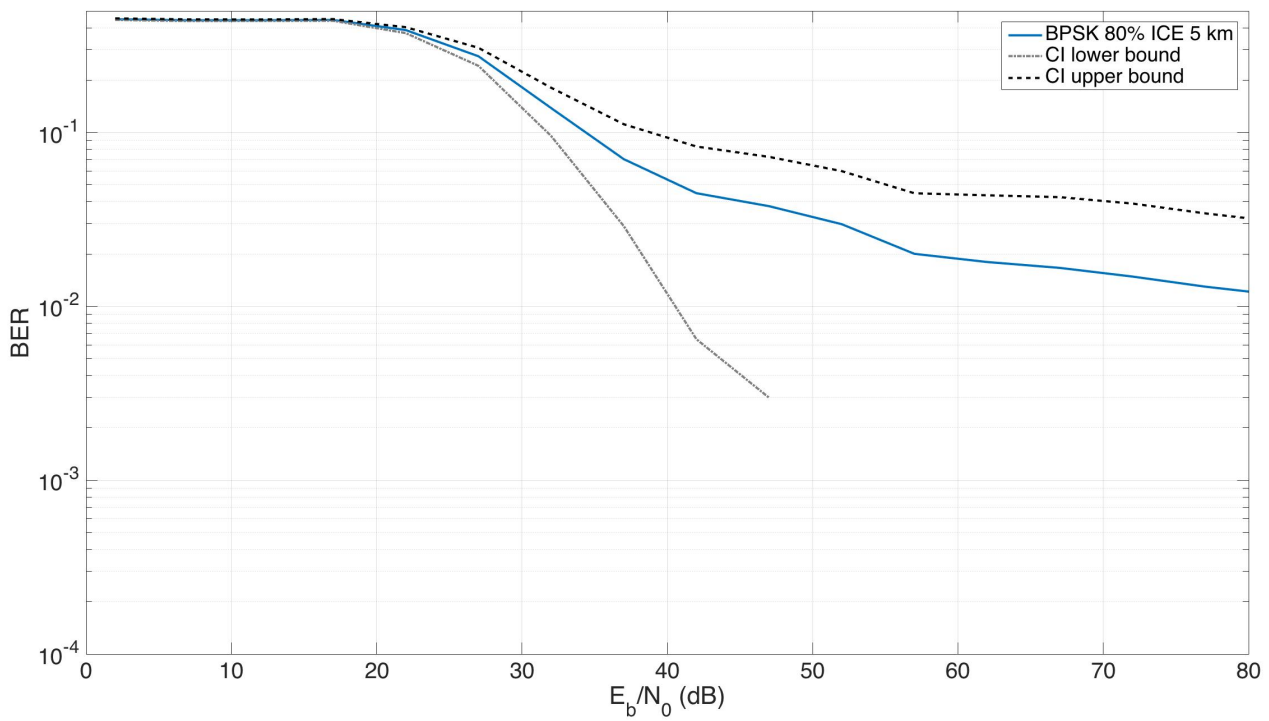

Figure 73: Confidence interval with 90\% confidence level. BPSK with $80 \%$ of ice cover, $5 \mathrm{~km}$ range and symbol time of three seconds. 


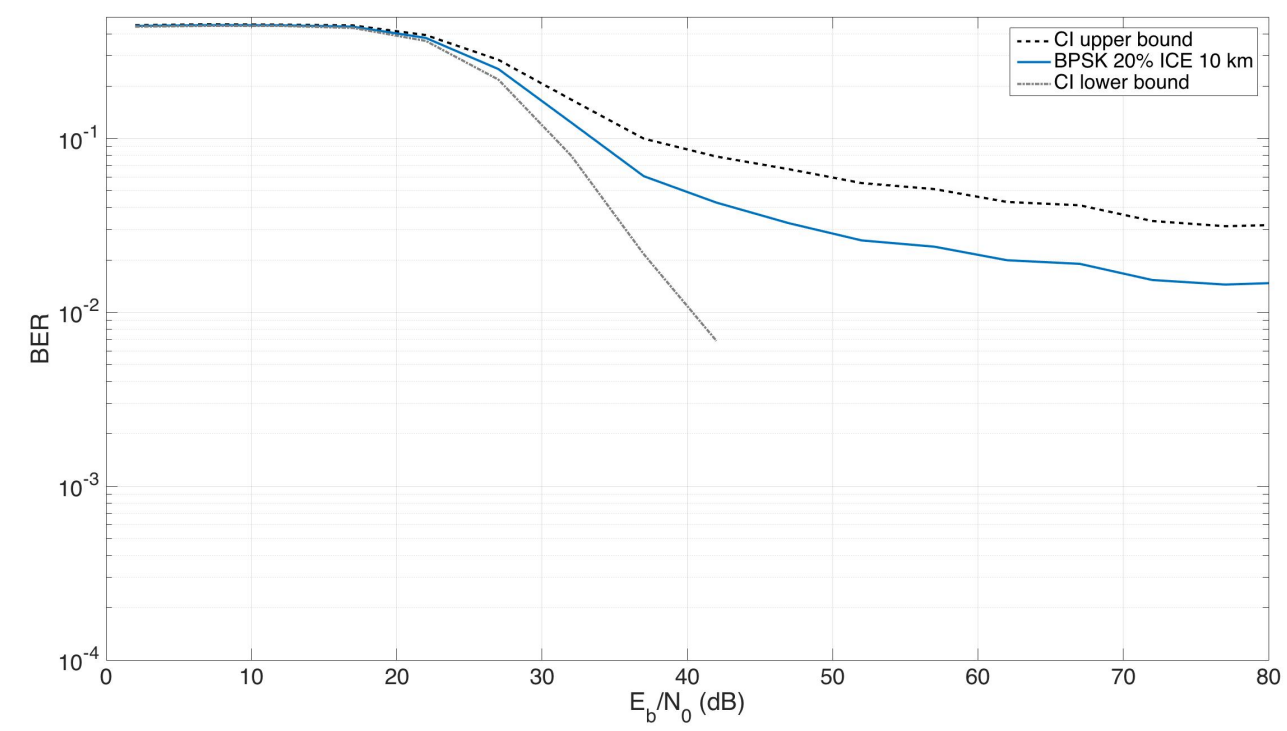

Figure 74: Confidence interval with 90\% confidence level. BPSK with $20 \%$ of ice cover, $10 \mathrm{~km}$ range and symbol time of three seconds. 
The following Figures show the BER confidence intervals for 2-FSK simulations.

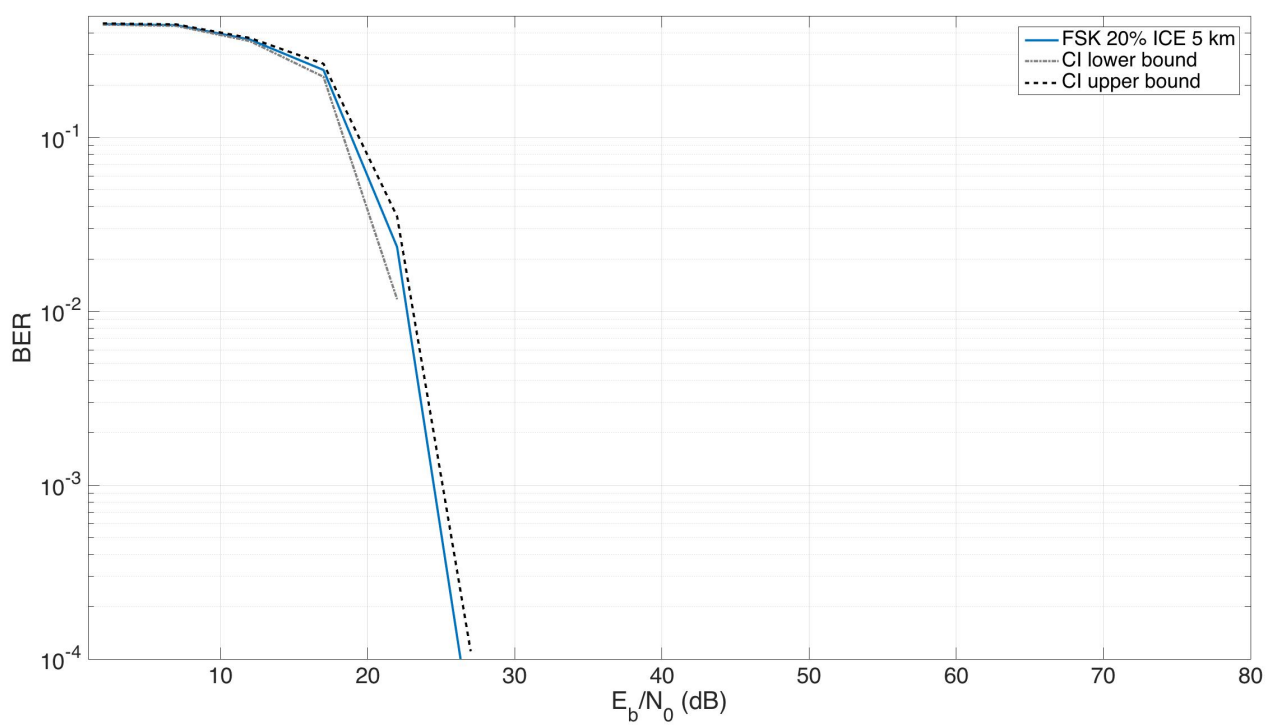

Figure 75: Confidence interval with $90 \%$ confidence level. 2-FSK with $20 \%$ of ice cover, $5 \mathrm{~km}$ range and symbol time of three seconds.

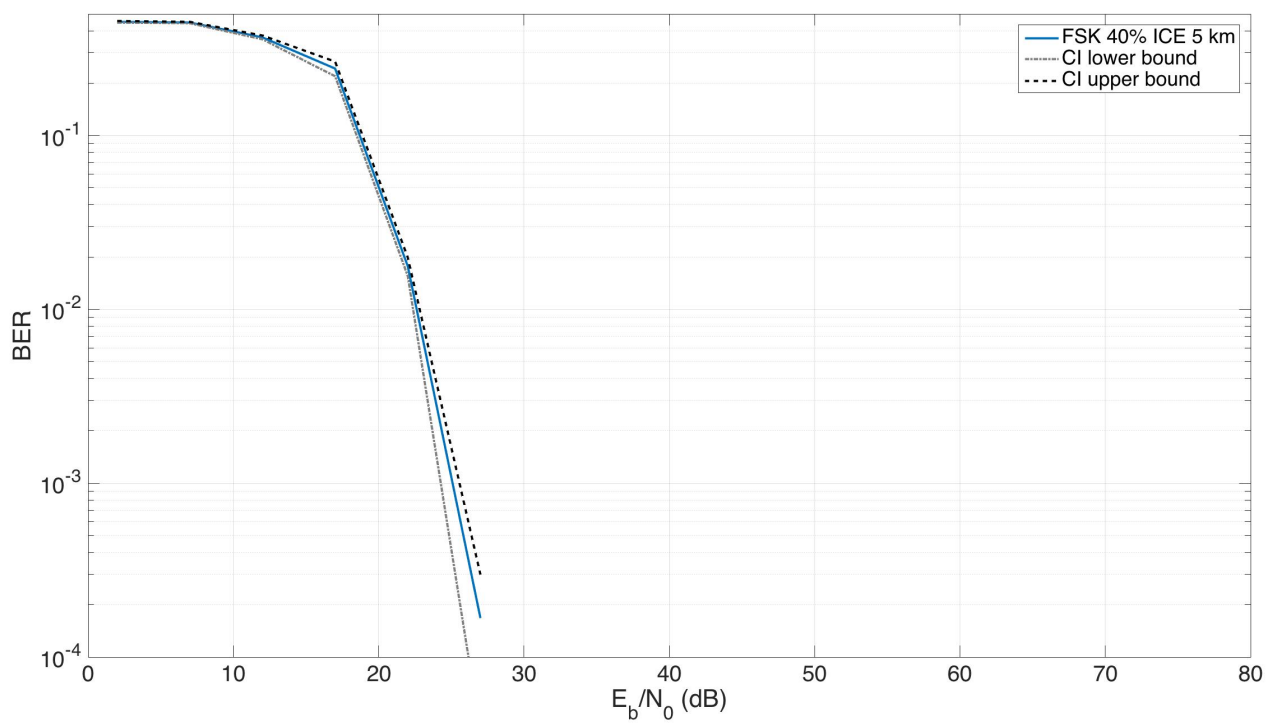

Figure 76: Confidence interval with $90 \%$ confidence level. 2-FSK with $40 \%$ of ice cover, $5 \mathrm{~km}$ range and symbol time of three seconds. 


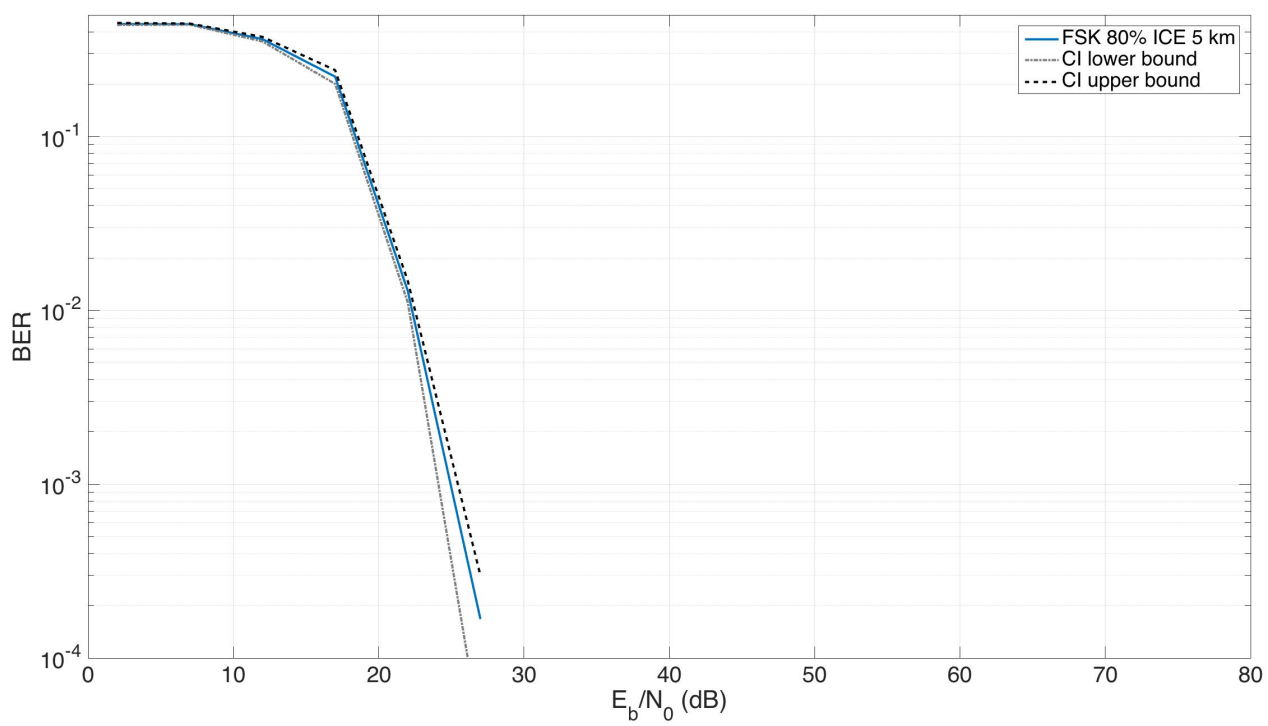

Figure 77: Confidence interval with $90 \%$ confidence level. 2-FSK with $80 \%$ of ice cover, $5 \mathrm{~km}$ range and symbol time of three seconds.

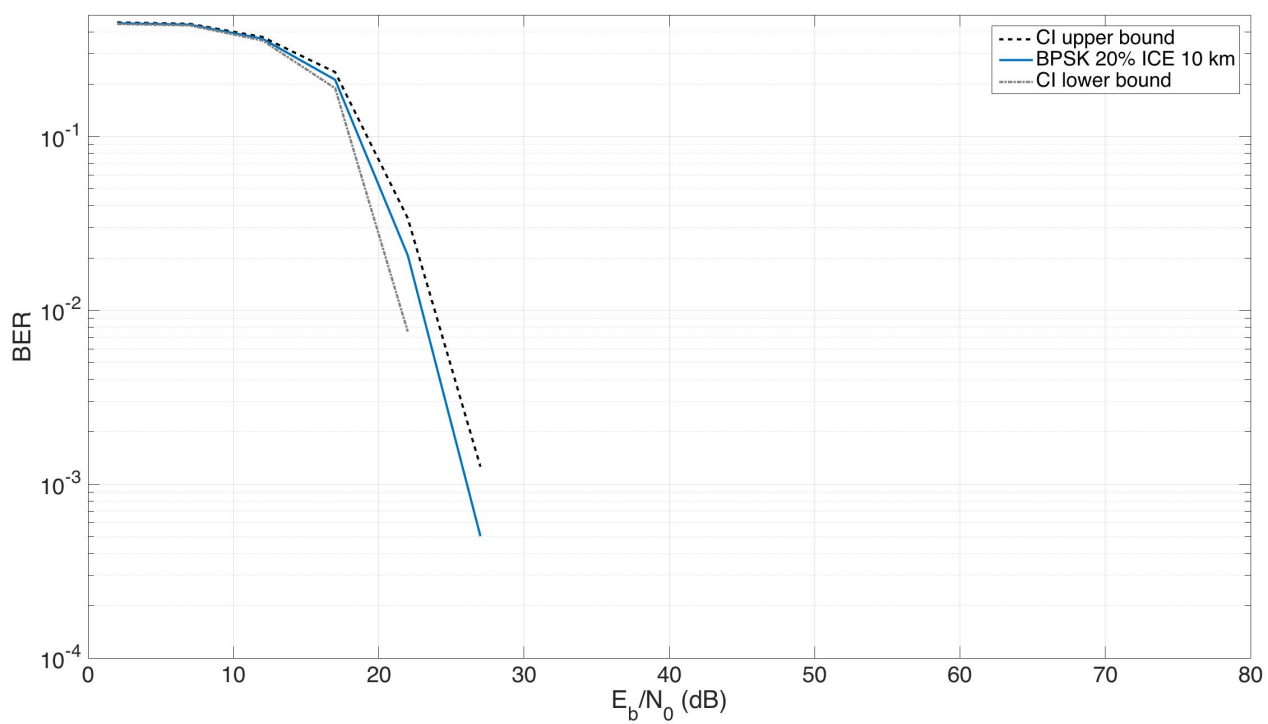

Figure 78: Confidence interval with $90 \%$ confidence level. 2-FSK with $20 \%$ of ice cover, $10 \mathrm{~km}$ range and symbol time of three seconds. 
The following Figures show the BER confidence intervals for FH-CPFSK simulations.

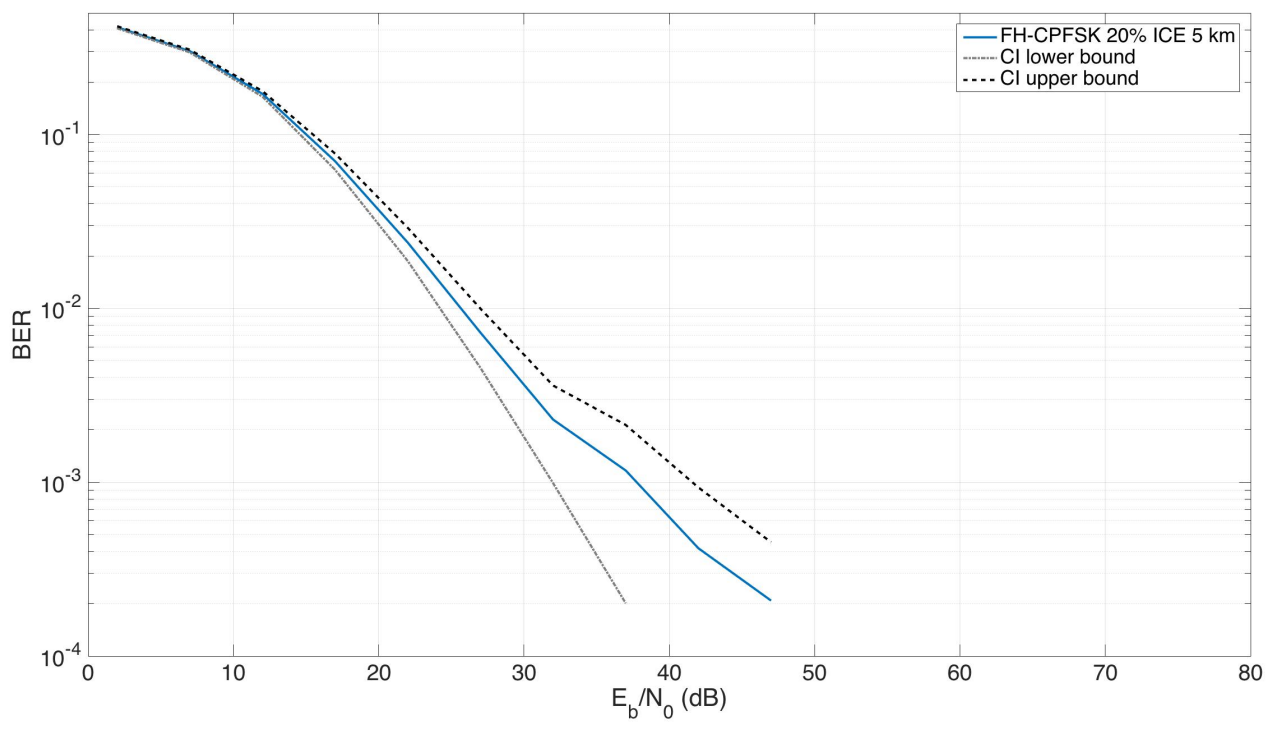

Figure 79: Confidence interval with $90 \%$ confidence level. FH-CPFSK with $20 \%$ of ice cover, $5 \mathrm{~km}$ range and symbol time of three seconds.

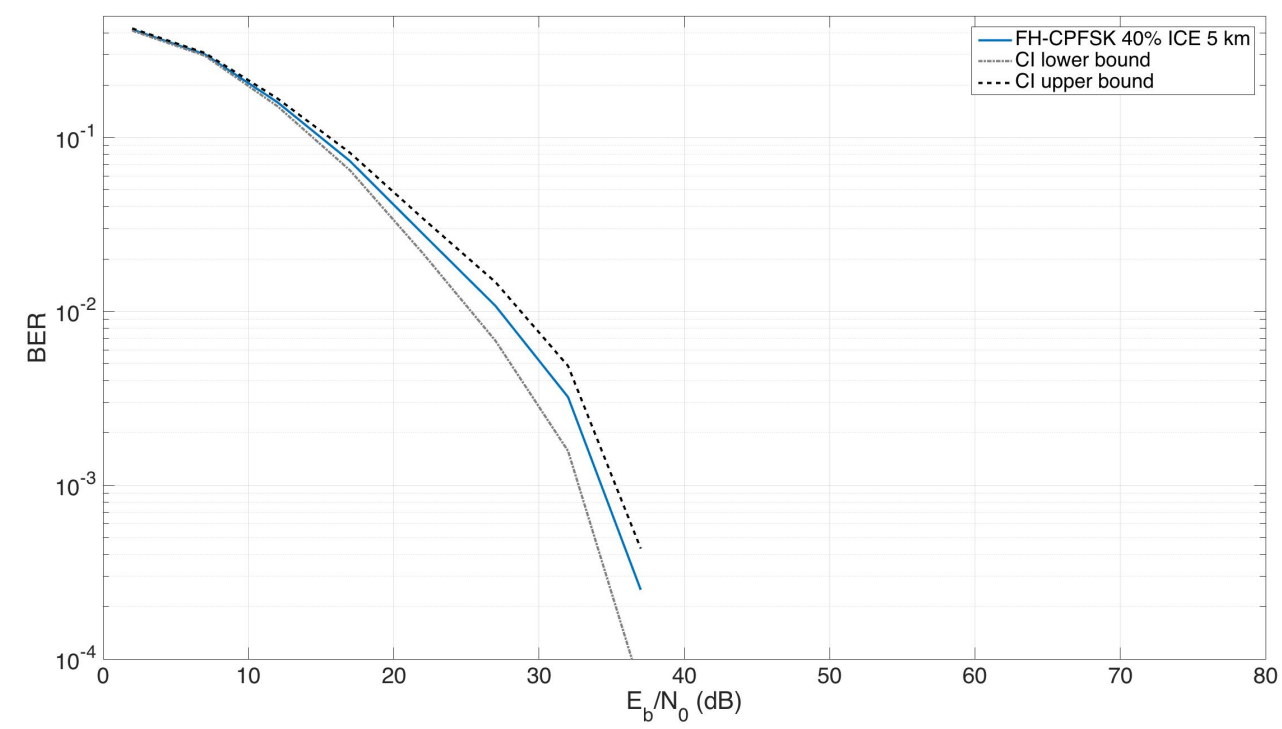

Figure 80: Confidence interval with $90 \%$ confidence level. FH-CPFSK with $40 \%$ of ice cover, $5 \mathrm{~km}$ range and symbol time of three seconds. 


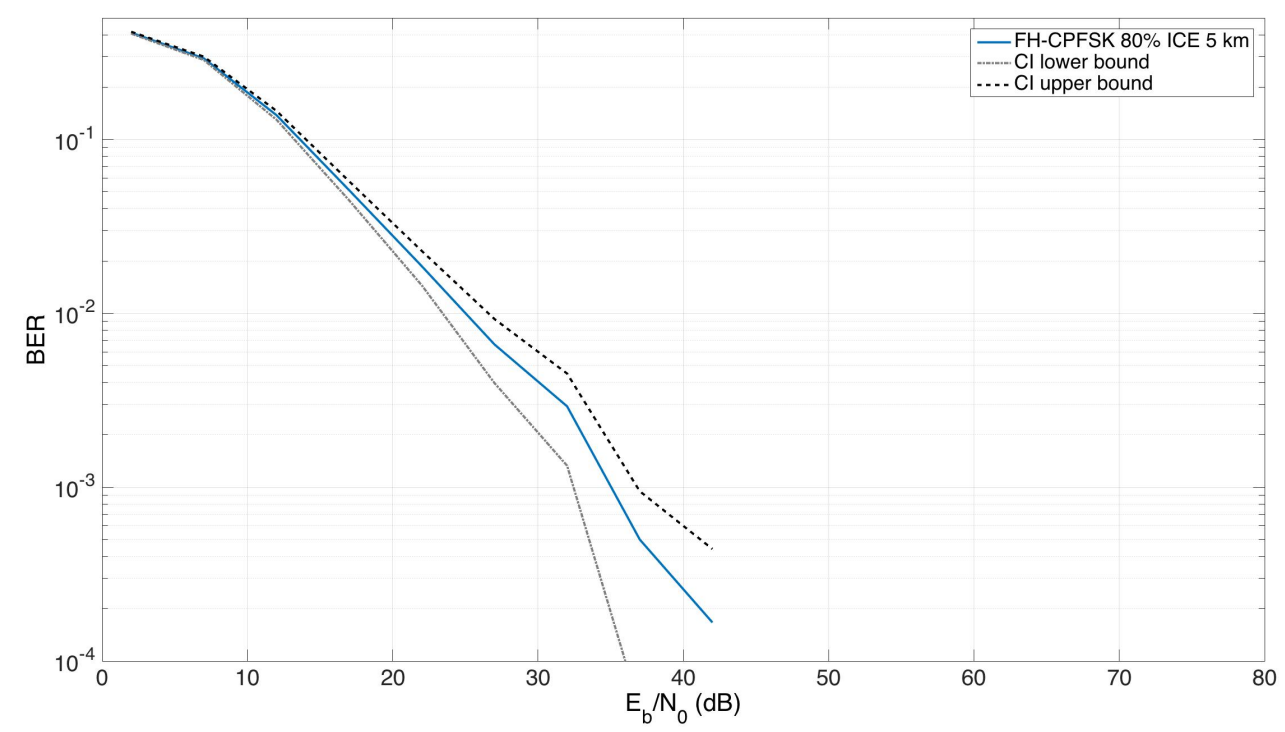

Figure 81: Confidence interval with $90 \%$ confidence level. FH-CPFSK with $80 \%$ of ice cover, $5 \mathrm{~km}$ range and symbol time of three seconds.

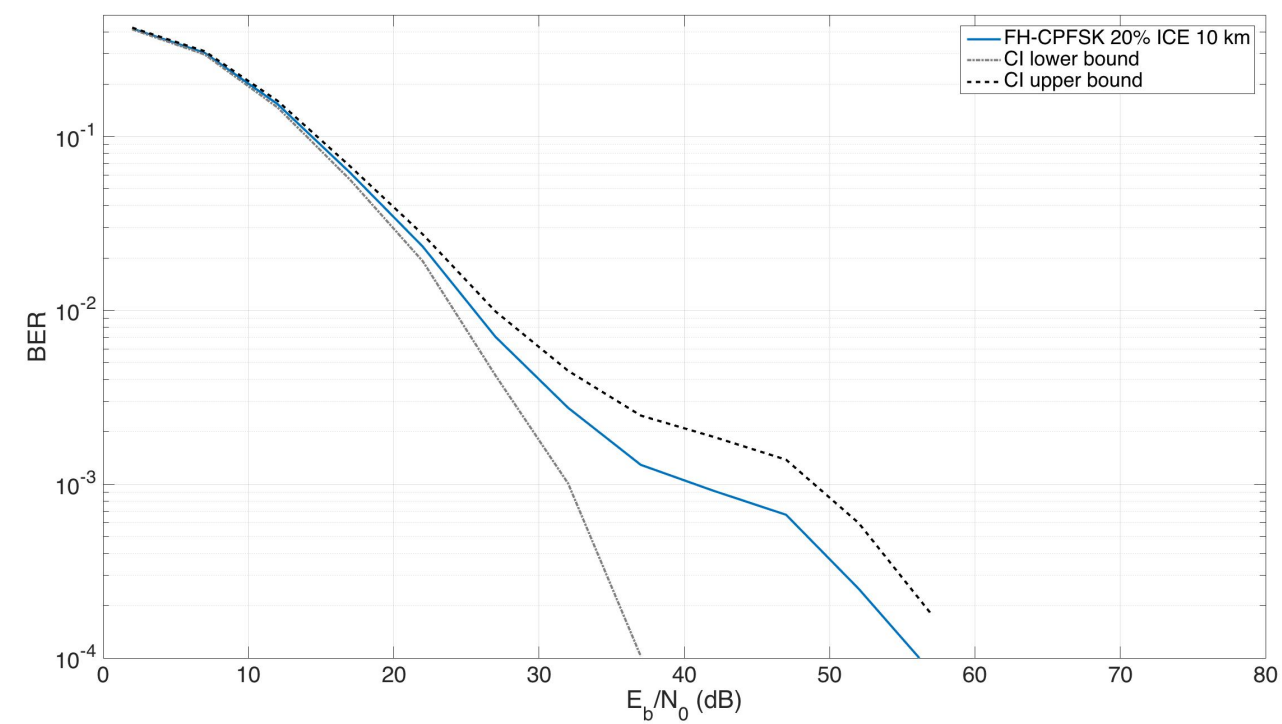

Figure 82: Confidence interval with $90 \%$ confidence level. FH-CPFSK with $20 \%$ of ice cover, $10 \mathrm{~km}$ range and symbol time of three seconds. 
The following Figures show the BER confidence intervals for O4Bp OFDM simulations.

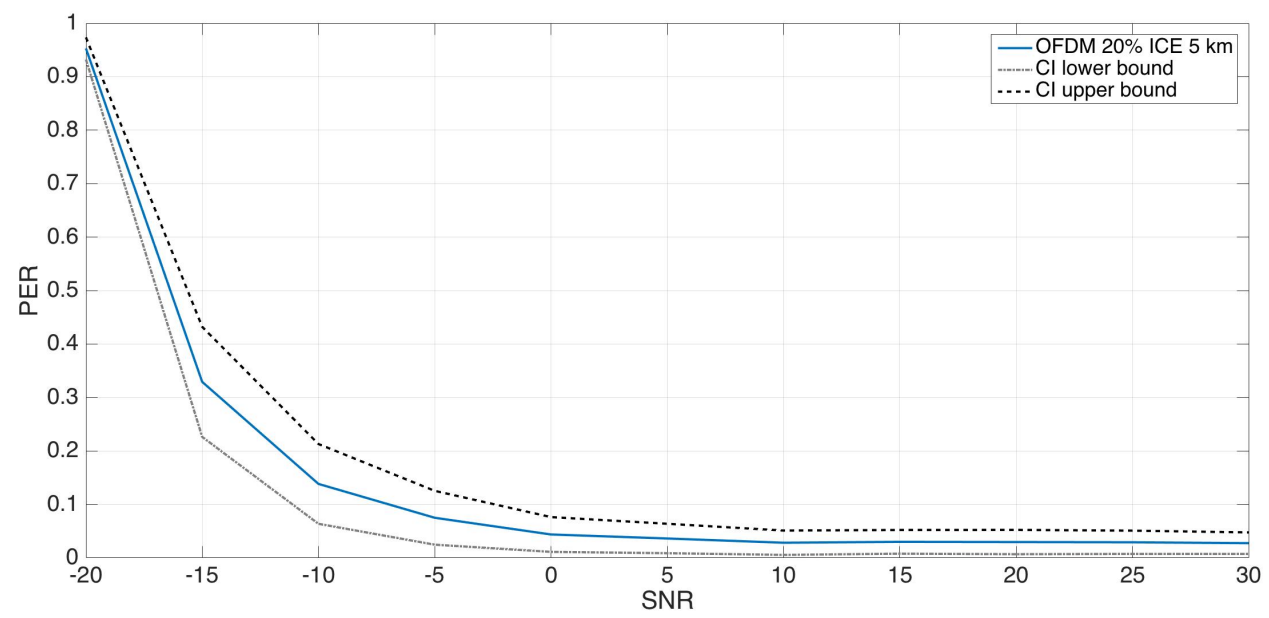

Figure 83: Confidence interval with 90\% confidence level. O4Bp OFDM with 20\% of ice cover and $5 \mathrm{~km}$ range.

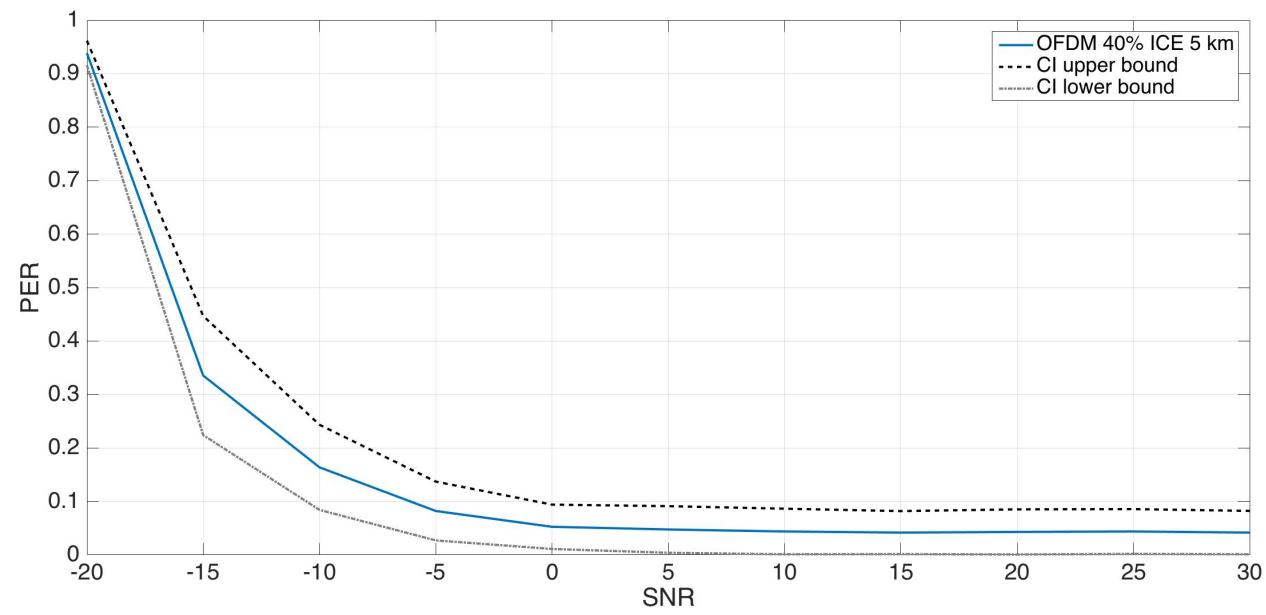

Figure 84: Confidence interval with 90\% confidence level. O4Bp OFDM with $40 \%$ of ice cover and $5 \mathrm{~km}$ range. 


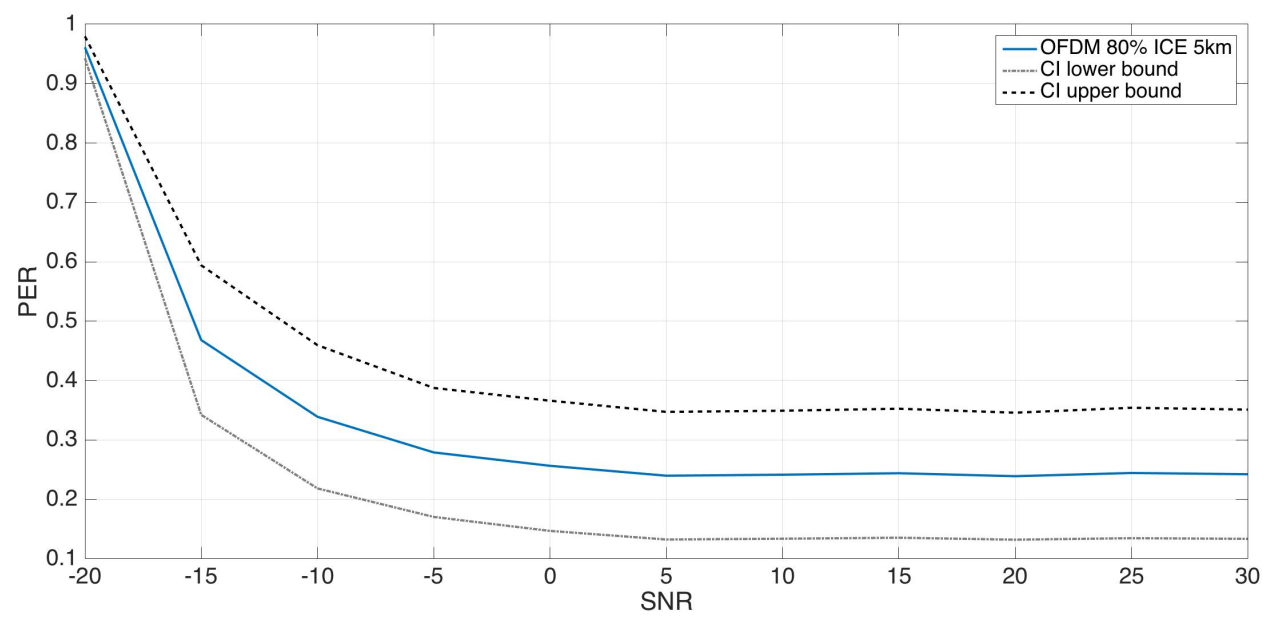

Figure 85: Confidence interval with $90 \%$ confidence level. O4Bp OFDM with $80 \%$ of ice cover and $5 \mathrm{~km}$ range.

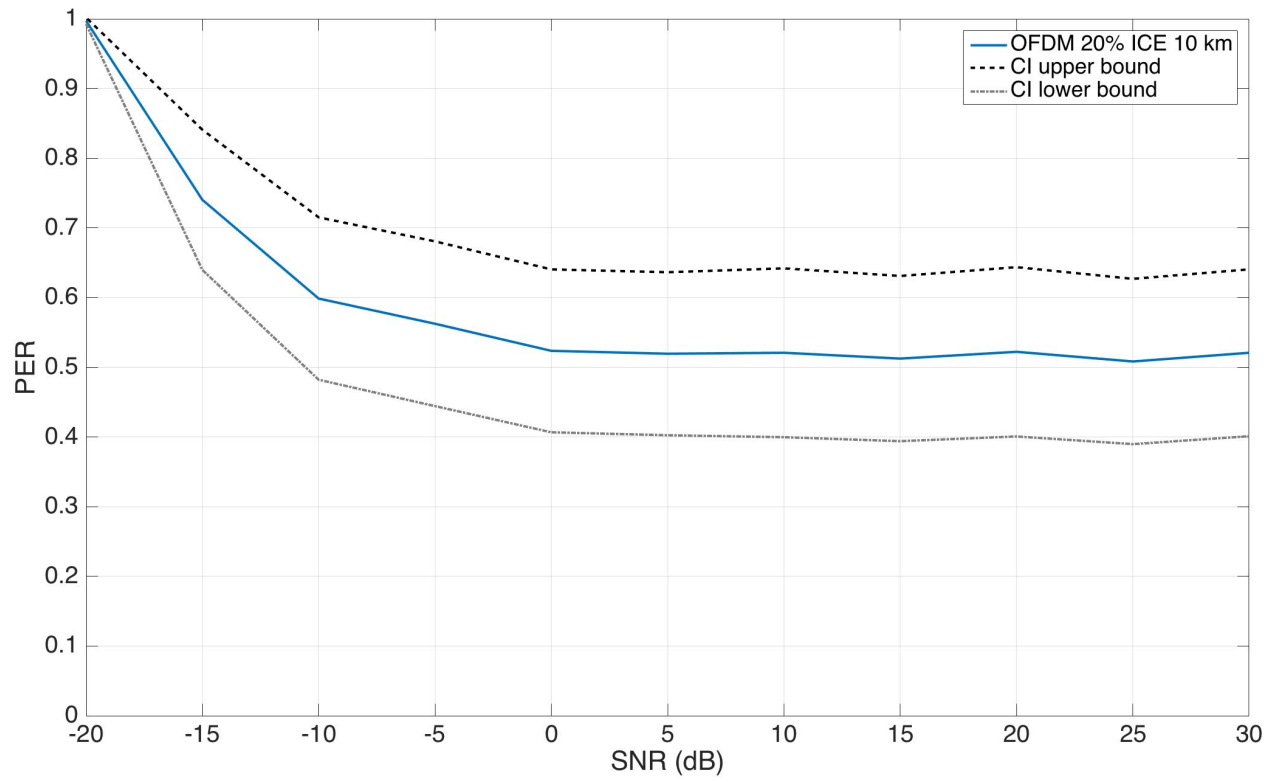

Figure 86: Confidence interval with $90 \%$ confidence level. O4Bp OFDM with $20 \%$ of ice cover and $10 \mathrm{~km}$ range. 


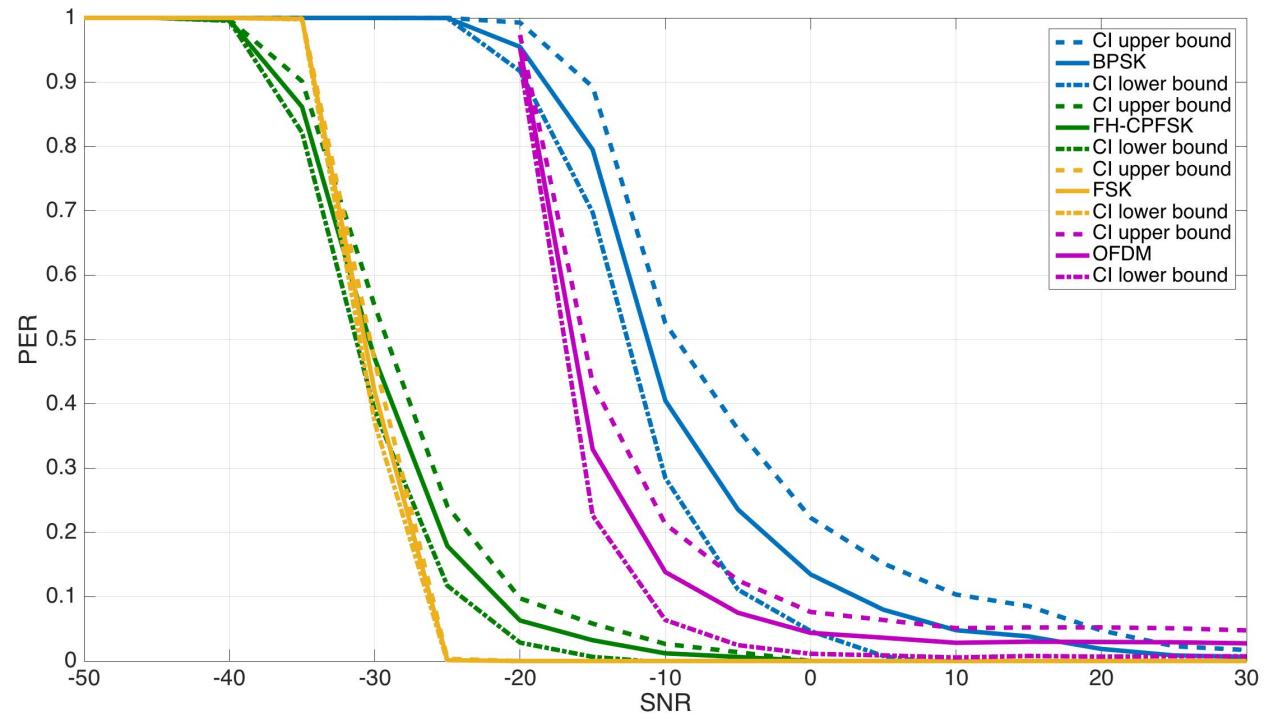

Figure 87: Confidence interval with 90\% confidence level. BPSK vs FSK vs FH-CFSK vs O4Bp OFDM in $20 \%$ of ice cover over a range of $5 \mathrm{~km}$ environment.

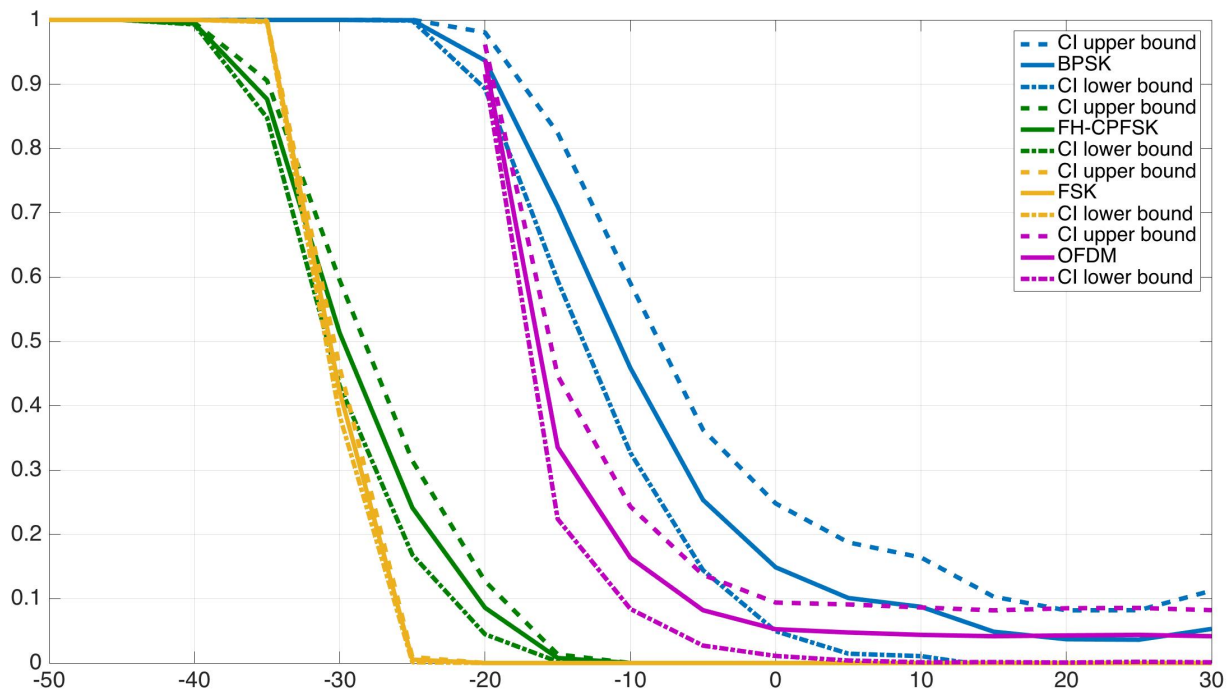

Figure 88: Confidence interval with 90\% confidence level. BPSK vs FSK vs FH-CFSK vs O4Bp OFDM in $40 \%$ of ice cover over a range of $5 \mathrm{~km}$ environment. 


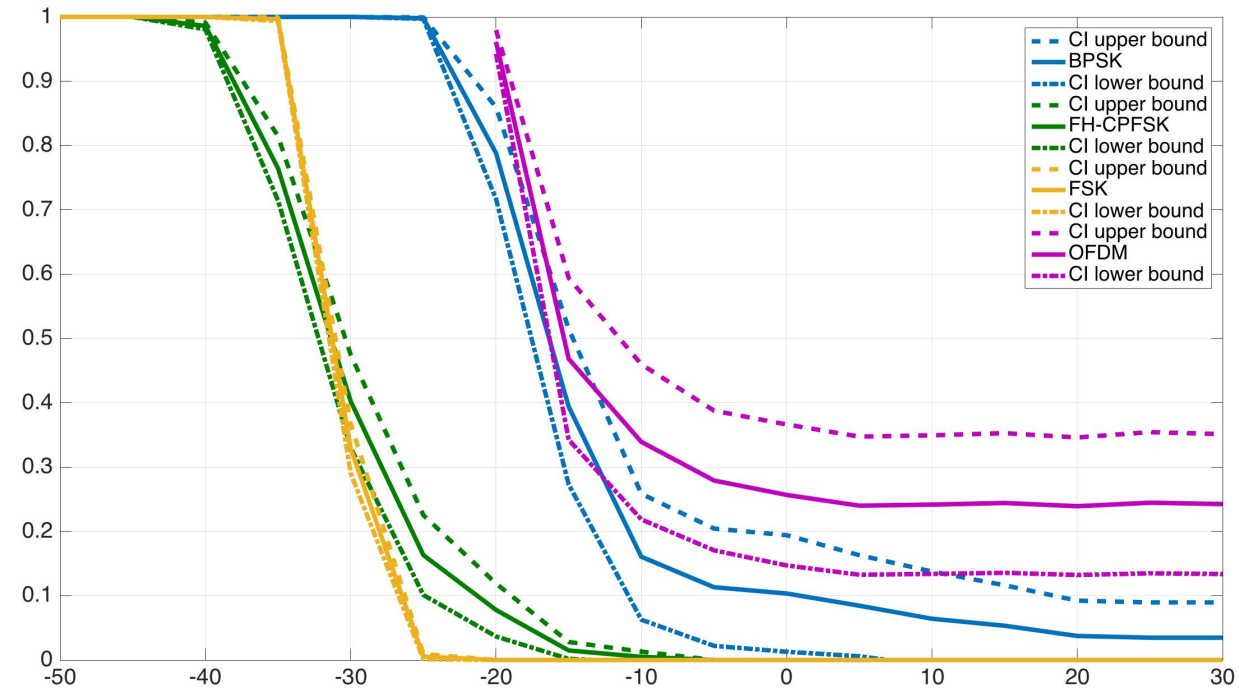

Figure 89: Confidence interval with 90\% confidence level. BPSK vs FSK vs FH-CFSK vs O4Bp OFDM in $80 \%$ of ice cover over a range of $5 \mathrm{~km}$ environment.

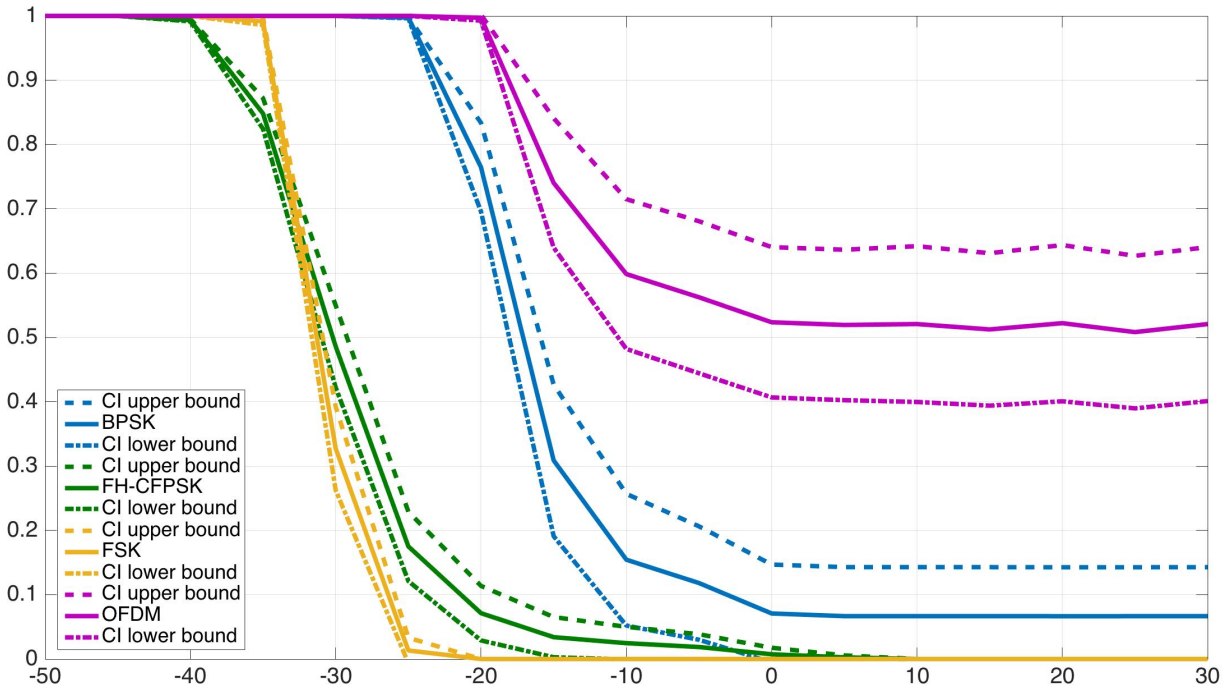

Figure 90: Confidence interval with 90\% confidence level. BPSK vs FSK vs FH-CFSK vs O4Bp OFDM in $20 \%$ of ice cover over a range of $10 \mathrm{~km}$ environment. 
Table A.1: Summary of Simulations Variables.

\begin{tabular}{|l|l|l|l|l|}
\hline & Data Rate & Bandwidth & Sampling Rate & Samples Per Symbol (with interpolation) \\
\hline BPSK $\left(T_{s}=1 / 3\right.$ second $)$ & $3 \mathrm{bps}$ & $4 \mathrm{~Hz}$ & $48,000 \mathrm{kHz}$ & 16,000 \\
\hline BPSK $\left(T_{s}=1\right.$ second $)$ & $1 \mathrm{bps}$ & $1.35 \mathrm{~Hz}$ & $48,000 \mathrm{kHz}$ & 48,000 \\
\hline BPSK $\left(T_{s}=3\right.$ seconds $)$ & $(1 / 3) \mathrm{bps}$ & $0.5 \mathrm{~Hz}$ & $48,000 \mathrm{kHz}$ & 144,000 \\
\hline FSK $\left(T_{s}=1 / 3\right.$ second) & $3 \mathrm{bps}$ & $10.6 \mathrm{~Hz}$ & $48,000 \mathrm{kHz}$ & 16,000 \\
\hline FSK $\left(T_{s}=1\right.$ second $)$ & $1 \mathrm{bps}$ & $3.5 \mathrm{~Hz}$ & $48,000 \mathrm{kHz}$ & 48,000 \\
\hline FSK $\left(T_{s}=3\right.$ seconds $)$ & $(1 / 3) \mathrm{bps}$ & $1.2 \mathrm{~Hz}$ & $48,000 \mathrm{kHz}$ & 144,000 \\
\hline FH-CPFSK $\left(T_{s}=1 / 3\right.$ second) & $3 \mathrm{bps}$ & $11261 \mathrm{~Hz}$ & $48,000 \mathrm{kHz}$ & 16,000 \\
\hline FH-CPFSK $\left(T_{s}=1\right.$ second $)$ & $1 \mathrm{bps}$ & $11254 \mathrm{~Hz}$ & $48,000 \mathrm{kHz}$ & 48,000 \\
\hline FH-CPFSK $\left(T_{s}=3\right.$ seconds $)$ & $(1 / 3) \mathrm{bps}$ & $11252 \mathrm{~Hz}$ & $48,000 \mathrm{kHz}$ & 144,000 \\
\hline O4Bp OFDM $\left(T_{s}=3\right.$ seconds $)$ & $2 \mathrm{bps}$ & $192 \mathrm{~Hz}$ & $48,000 \mathrm{kHz}$ & $144,000(2250$ per carrier) (total 64 carriers) \\
\hline
\end{tabular}




\section{Bibliography}

[1] C. Jones and A. Morozov, "Under ice positioning and communications for unmanned vehicles," in 2013 MTS/IEEE OCEANS - Bergen. IEEE, 2013, pp. $1-6$.

[2] L. Freitag, K. Ball, J. Partan, P. Koski, and S. Singh, "Long range acoustic communications and navigation in the arctic," in OCEANS 2015 - MTS/IEEE Washington. MTS, 2015, pp. 1-5.

[3] "WHOI Modems," http://acomms.whoi.edu/micro-modem/, [Online; accessed August-2016].

[4] "EVOLOGIC Modems," https://www.evologics.de/en/products/acoustics/ index.html, [Online; accessed August-2016].

[5] "DEVOLOGIC Modems," http://www.develogic.de/products/underwatercommunication-systems, [Online; accessed August-2016].

[6] "TELEDYNE Modems," http://teledynebenthos.com/product_dashboard/ acoustic_modems, [Online; accessed August-2016].

[7] J. Heidemann, M. Stojanovic, and M. Zorzi, "Underwater sensor networks: applications, advances and challenges," Philosophical Transactions: Mathematical, Physical and Engineering Sciences, vol. 370, pp. 158-175, January 2012.

[8] L. Lanbo, Z. Shengli, and C. Jun-Hong, "Prospects and problems of wireless communication for underwater sensor networks," Wireless Communications and Mobile Computing, vol. 8, pp. 977 - 994, October 2008.

[9] M. Lanzagorta, Underwater communications, 1st ed., ser. Synthesis lectures on communications. Morgan \& Claypool, 2013, vol. 6, no. 27-40.

[10] J. G. Proakis, Digital Communications, 4th ed. McGrawHill, 2000.

[11] S. Zhou and Z. Wang, OFDM for underwater acoustic communications. John Wiley \& Sons Ltd, 2014.

[12] P. Ozog, M. Leeser, and M. Stojanovic, "Adapting the USRP as an underwater acoustic modem," High Performance Embedded Computing, 2009.

[13] M. Dillinger, K. Madani, and N. Alonistioti, Software Defined Radio: Architectures, Systems and Functions. Wiley, 2005. 
[14] B. S. Borowski, "Application of channel estimation to underwater acoustic communication," Ph.D. dissertation, Stevens Institute of Technology, 2010.

[15] M. B. Porter, The BELLHOP Manual and User's Guide, January 2011.

[16] C. Hamm, S. Blouin, and M. Taillefer, "Toward hardware-driven simulation of underwater acoustic propagation and communication with arctic ice, wind waves, and currents," in OCEANS 2015 - MTS/IEEE Washington. IEEE, October 2015, pp. 1-6.

[17] F. B. Jensen, W. A. Kuperman, M. B. Porter, and H. Schmidt, Modern Acoustics and Signal Processing: Computational Ocean Acoustics (2nd Edition), 2011.

[18] R. Istepanian and M. Stojanovic, Eds., Underwater Acoustic Digital Signal Processing and Communication Systems. Springer US, 2002.

[19] M. Barbeau, Wireless Mobile Communications, Networks \& Security. Draft manuscript, 2015.

[20] L. Brekhovskikh and Y. Lysanov, Fundamentals of ocean acoustics, 3rd ed. Springer-Verlag, 2003.

[21] "Discovery of Sound in the Sea," http://www.dosits.org/science/ soundsinthesea/commonsounds/, 2016, [Online; accessed August-2016].

[22] "Underwater acoustic communication," http://web.mit.edu/millitsa/www/ resources/pdfs/ency.pdf, [Online; accessed August-2016].

[23] F. Dowla, Handbook of RF and wireless technologies, 1st ed. Newnes, 2004.

[24] "Software Defined Radio," http://www.wirelessinnovation.org/assets/ documents/SoftwareDefinedRadio.pdf, [Online; accessed August-2016].

[25] "GNU Radio Guided Tutorials," "http://gnuradio.org/redmine/projects/ gnuradio/wiki/Guided_Tutorials", [Online; accessed August-2016].

[26] "OSI Stack," "https://en.wikipedia.org/wiki/OSI_model", [Online; accessed August-2016].

[27] E. Demirors, G. Sklivanitis, T. Melodia, S. N. Batalama, and D. A. Pados, "Software-defined underwater acoustic networks: Toward a high-rate real-time reconfigurable modem," IEEE Communications Magazine, vol. 53, no. 11, pp. 64-71, 112015.

[28] L. Wu, J. Trezzo, D. Mirza, P. Roberts, J. Jaffe, Y. Wang, and R. Kastner, "Designing an adaptive acoustic modem for underwater sensor networks," IEEE Embedded Systems Letters, vol. 4, pp. 1-4, 2012. 
[29] B. Borowski and D. Duchamp, "The softwater modem: a software modem for underwater acoustic communication: short paper," in Proceedings of the Fourth ACM International Workshop on underwater networks, ser. WUWNet '09. ACM, November 2009.

[30] C. Gianni, C. Alessandro, L. Marco, and R. Claudio, "Seamodem: A low-cost underwater acoustic modem for shallow water communication," in OCEANS 2015 - Genova. IEEE, 2015, pp. 1-6.

[31] B. Benson, Y. Li, B. Faunce, K. Domond, D. Kimball, C. Schurgers, and R. Kastner, "Design of a low-cost underwater acoustic modem," IEEE Embedded Systems Letters, vol. 2, no. 3, September 2010.

[32] P. van Walree, H. Buen, and R. Otnes, "A performance comparison between dsss, $\mathrm{m}$-fsk, and frequency-division multiplexing in underwater acoustic channels," in 2014 Underwater Communications and Networking (UComms). IEEE, 2014, pp. $1-5$.

[33] H. Dol, P. Casari, and T. van der Zwan, "2014 underwater communications and networking (ucomms)." IEEE, 2014, pp. 1-5.

[34] "Network Simulator," "https://www.nsnam.org/", [Online; accessed August2016].

[35] S. Raza', "PSK Modulation and Demodulation on Matlab." https: //www.mathworks.com/matlabcentral/fileexchange/44822-matlab-code-forpsk-modulation-and-demodulation, [Online; accessed August-2016].

[36] "Interpolation," http://dspguru.com/dsp/faqs/multirate/interpolation, [Online; accessed August-2016].

[37] S. Raza', "FSK Modulation and Demodulation on MATLAB." https: //www.mathworks.com/matlabcentral/fileexchange/44821-matlab-code-forfsk-modulation-and-demodulation/content/FSK_mod_demod_salim.m, [Online; accessed August-2016].

[38] A. Goldsmith, Wireless Communications, 1st ed. Cambridge University Press, 2005.

[39] T. M. Schmidl and D. C. Cox, "Robust frequency and timing synchronization for ofdm," IEEE Transactions on Communications, 1997.

[40] P. David, "Spread Spectrum Techniques in GNU Radio," http: //static1.1.sqspcdn.com/static/f/679473/25461602/1411158960237/

Sep18_07_David_DSSSFHSS.pdf?token=Ivu9LTilloy432Jzj50m7ejcIXQ\%3D, 2014, [Online; accessed August-2016]. 
[41] P. David', "GR-SPREAD Module for GNU Radio," https://github.com/CIGSDR/CIG/tree/master/gr-spread, [Online; accessed August-2016].

[42] J. A. Goff, "Quantitative analysis of sea ice draft: 1. methods for stochastic modeling," Journal of Geophysical Research, vol. 100, no. C4, pp. 6993-7004, April 1995.

[43] J. Steele, S. Thorpe, and K. Turekian, Elements of Physical Oceanography, 1st Edition. Academic Press, 2010.

[44] "InterQuartile Range," "https://en.wikipedia.org/wiki/Interquartile_range", [Online; accessed August-2016].

[45] "GNU Radio PSK Demodulation," gnuradio.org/redmine/projects/gnuradio/ wiki/Guided_Tutorial_PSK_Demodulation, [Online; accessed August-2016].

[46] J. Huang, "Simulation and Modelling of Underwater acoustic communication Channels with Wide Band Attenuation and Ambient Noise," Master's thesis, Carleton University, 2015.

[47] "FIR," http://dspguru.com/dsp/faqs/fir/basics, [Online; accessed August-2016].

[48] "FSK Transmitter," https://nccgroup.github.io/RFTM/fsk_transmitter.html, [Online; accessed August-2016].

[49] M. J. Crocker, Ed., Handbook of Acoustics. Wiley, 1998.

[50] "Pulse-Shape Filtering in Communications Systems," http://www.ni.com/ white-paper/3876/en/ $\$ toc7, [Online; accessed August-2016].

[51] H. Malepati, Digital Media Processing: DSP Algorithms Using C. Newnes, 2010.

[52] M. Barbeau, Software Defined Radio Wireless Communications The Software Way!, 2015. http://people.scs.carleton.ca/ barbeau/SDRCRBook/index.shtml.

[53] "Underwater Simulation for Several Modulation Techniques -Github link," https://github.com/ahmadtraboulsi/UW_modulation_schemes, [Online; accessed August-2016]. 